\begin{tabular}{|l|l|}
\hline $\begin{array}{l}\text { 2. To: (Receiving Organization) } \\
\text { Distribution }\end{array}$ & $\begin{array}{l}\text { 3. From: (Originating Organization) } \\
\text { Spent Nuclear Fuel } \\
\text { Evaluat ions/8M710 }\end{array}$ \\
\hline $\begin{array}{l}\text { 5. Proj./Prog./Dept./Div.: } \\
\text { Spent Nuclear Fuel Project }\end{array}$ & $\begin{array}{l}\text { 6. Design Authority/ Design Agent/Cog. } \\
\text { S.L.'. Hecht }\end{array}$ \\
\hline
\end{tabular}

8. Originator Remarks:

For approval and information--ASSESSMENT OF THE QUANTITIES AND DISTRIBUTION OF $K$ EAST BASIN FLOOR SLUDGE CONSTITUENTS

11. Receiver Remarks: 11A. Design Baseline Document? [ ] Yes. [X] No
4. Related EDT No.:

7. Purchase Order No.:

$$
\text { N/A }
$$

9. Equip./Component No.:

$$
\text { N/A }
$$

10. System/Bldg./Facility: 105-K East

12. Major Assm. Dwg. No.:

$$
\text { N/A }
$$

13. Permit/Permit Application No.: $N / A$

14. Required Response Date:

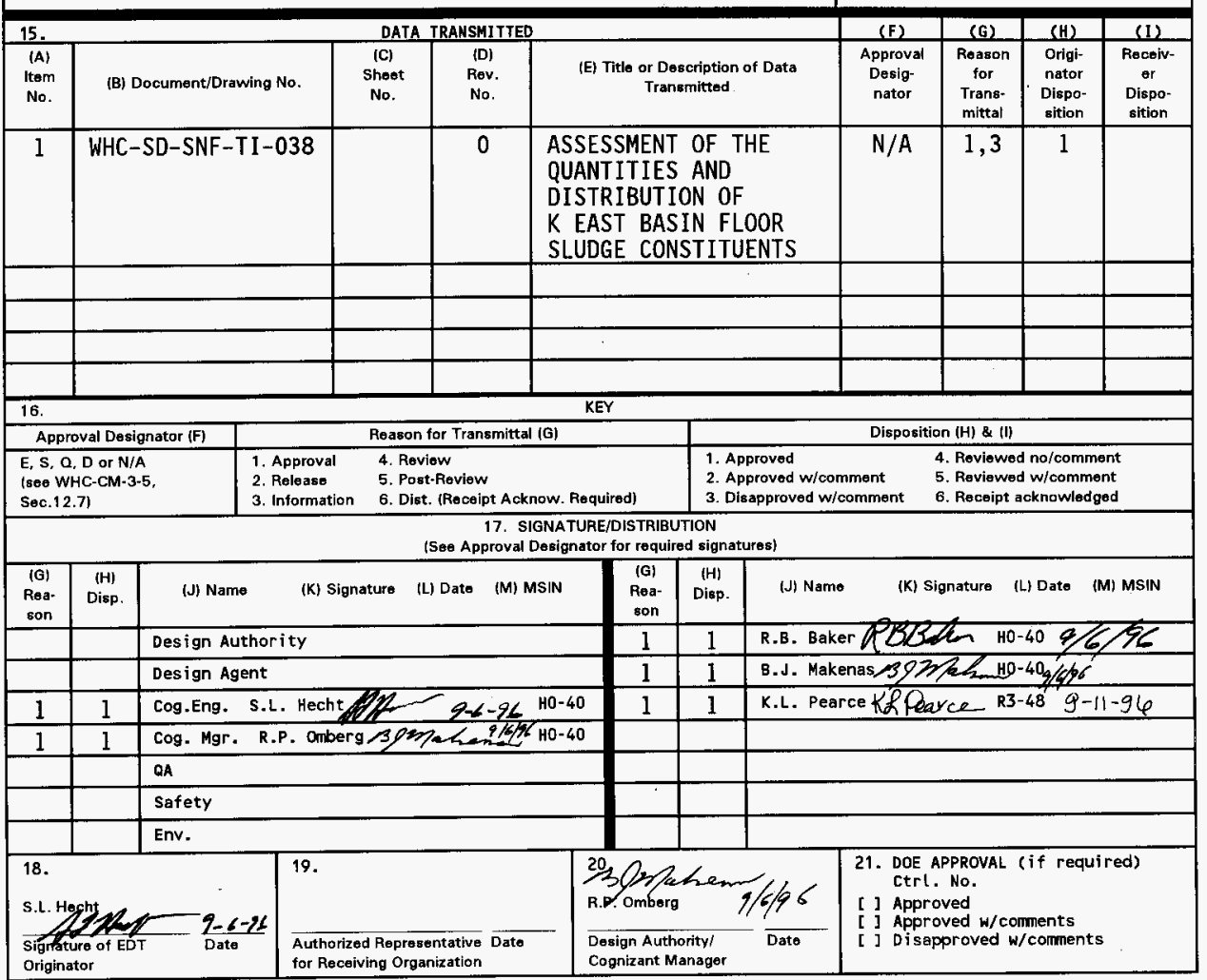




\section{ASSESSMENT OF THE QUANTITIES AND DISTRIBUTION OF K EAST BASIN FLOOR SLUDGE CONSTITUENTS}

S. L. Hecht

West inghouse Hanford Company, Richland, WA 99352

U.S. Department of Energy Contract DE-AC06-87RL10930

$\begin{array}{llll}\text { EDT/ECN: } & 617436 & \text { UC: } 2070 & \\ \text { Org Code: } & 8 M 710 & \text { Charge Code: } & \text { LBO29 } \\ \text { B\&R Code: } & \text { EW } 3135040 & \text { Total Pages: } & 134\end{array}$

Key Words: K Basin, Sludge, Characterization, Analysis, and Spent Nuclear Fuel Project

Abstract: Floor sludge constituent masses and their spatial

distribution within $\mathrm{K}$ East Basin were calculated based on results from recent laboratory chemical and radionuclides analyses of sludge sampled in $K$ East main basin along with data previously generated from the analysis of measured sludge depths.

Results of these calculations, showed that the major gravimetric constituents of the sludge are iron, uranium, aluminum, sodium, and calcium. Significant amounts of fissionable materials ${ }^{235} \mathrm{U}$ and ${ }^{239} \mathrm{Pu}$ were also calculated. The calculated distribution of sludge constituent masses showed distinct patterns.

TRADEMARK DISCLAIMER. Reference herein to any specific comercial product, process, or service by trade name, trademark, manufacturer, or otherwise, does not necessarily constitute or imply its endorsement, recommendation, or favoring by the United States Government or any agency thereof or its contractors or subcontractors.

Printed in the United states of America. To obtain capies of this document, contact: WHC/BCS Document Control Services, P.O. Box 1970, Mailstop H6-08, Richland HA 99352, Phone (509) 372-2420; Fax (509) 376-4989.
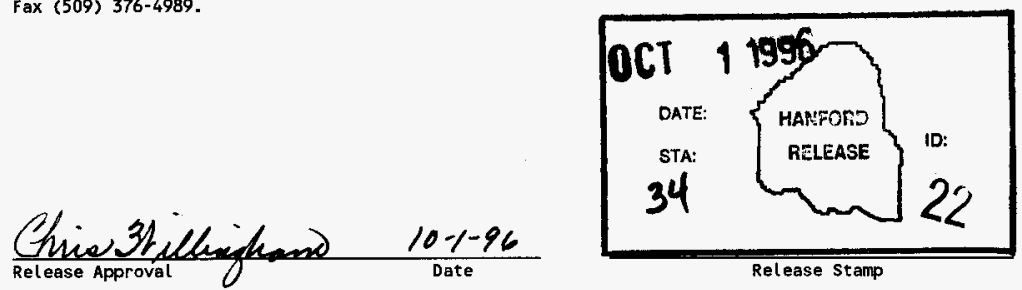

Release Stamp 
Trademark Listing

SUN is a trademark of Sun Microsystems 
WHC-SD-SNF-TI-038, Rev. 0

\section{ASSESSMENT OF THE QUANTITIES AND DISTRIBUTION OF \\ $K$ EAST BASIN FLOOR SLUDGE CONSTITUENTS}

S. L. Hecht

Spent Nuclear Fuel Evaluations

September 1996 
WHC-SD-SNF-TI-038, Rev. 0

\section{CONTENTS}

1.0 SUMMARY AND CONCLUSIONS . . . . . . . . . . . . . . 3

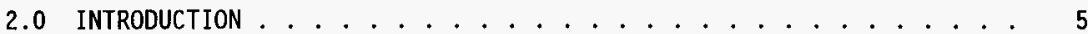

3.0 ANALYSES AND RESULTS . . . . . . . . . . . . 7

3.1 ANALYSIS OF SLUDGE CONSTITUENTS CONCENTRATIONS . . . . . . 7

3.2 ANALYSIS OF SLUDGE CONSTITUENTS MASSES . . . . . . . . . 10

4.0 DISCUSSION . . . . . . . . . . . . . . 13

4.1 UNCERTAINTIES . . . . . . . . . . . . . . . . . 13

4.2 COMPARISON WITH PREVIOUS PREDICTIONS . . . . . . . . 13

4.3 GENERAL DISCUSSION .......................... 14

5.0 ACKNOWLEDGEMENTS . . . . . . . . . . . . . . . 15

6.0 REFERENCES . . . . . . . . . . . . . . . . . 17

$\begin{array}{lll}\text { APPENDIX A } & \text { VISUALIZATION PLOTS OF CONCENTRATIONS OF SELECTED } \\ & \text { CONSTITUENTS FOR K EAST FLOOR SLUDGE . . . . . . . . } 19\end{array}$

APPENDIX B VISUALIZATION PLOTS OF FLOOR SLUDGE DEPTH DATA FROM K EAST . . . . . . . . . . . . . 71

APPENDIX $C$ VISUALIZATION PLOTS OF MASS DISTRIBUTIONS

OF SELECTED CONSTITUENTS FOR K EAST FLOOR SLUDGE . . . . 75

APPENDIX D COMPUTER CODE SOURCE LISTINGS . . . . . . . . . 123 
WHC-SD-SNF-TI-038, Rev. 0

This page intentionally left blank. 
WHC-SD-SNF-TI-038, Rev. 0

\section{ASSESSMENT OF THE QUANTITIES AND DISTRIBUTION OF \\ K EAST BASIN FLOOR SLUDGE CONSTITUENTS}

\subsection{SUMMARY AND CONCLUSIONS}

Floor sludge constituent masses and their spatial distribution within $K$ East Basin were calculated. This information will be beneficial in the assessing the acceptability of $\mathrm{K}$ Basin sludge in the Tank Waste Remediation System and in the design and/or operational practices related to retrieval and handling of this $K$ Basin sludge.

Calculations were made based on results from recent laboratory chemical and radionuclides analyses of sludge sampled from thirteen locations in $K$ East main basin. This concentration data was mathematically processed to determine the various constituents concentration distribution throughout the basin floor. This data was further processed, along with data previously generated from the analysis of measured sludge depths, to give both basin total masses and associated spatial distribution of the various sludge constituents.

Results of these calculations, showed that the major gravimetric constituents of the sludge are iron $(1505 \mathrm{~kg})$, uranium $(1387 \mathrm{~kg})$, aluminum, sodium, and calcium. Significant amounts of fissionable materials were al so calculated: ${ }^{235} \mathrm{U}(9.92 \mathrm{~kg})$ and ${ }^{239} \mathrm{Pu}(3.42 \mathrm{~kg})$.

The calculated distribution of sludge constituent masses showed distinct patterns. Sludge constituent associated with corroded spent nuclear fuel were concentrated near the north-west corner of the basin. Aluminum and iron, and many other elements are mainly located near the mouth of the South Load Out Pit. A value of $10,200 \mathrm{Ci}$ of alpha $(5 \%)+$ beta (95\%) radioactivity was calculated for the total basin floor sludge, and was concentrated near the north-west corner of the basin and near the Dummy Elevator Pit. 
WHC-SD-SNF-TI-038, Rev. 0

This page intentionally left blank. 
WHC-SD-SNF-TI-038, Rev, 0

\subsection{INTRODUCTION}

It is currently estimated (Baker 1995) that $21.7 \mathrm{~m}^{3}\left(765 \mathrm{ft}^{3}\right)$ of particulate layered material, generally referred to as sludge, has accumulated on the floor of K East Basin. This sludge is believed to be generated from mainly spalled corrosion products from spent metallic uranium fuel elements (both fuel and cladding), from fuel canisters (primarily aluminum ones), and from fuel canister storage racks. Additional sludge constituents are believed to come from the concrete basin walls, paint chips, ion-exchange resins, and wind blown debris such as sand.

Removal from $K$ Basin and interim safe storage of this sludge is a major part of the $K$ Basin cleanup effort. In order to safely and effectively accomplish this goal, characterization of the sludge chemical make-up is required. More specifically, the amounts and distribution of key chemical constituents within K East (KE) Basin need be determined. Development of methods and design of equipment for retrieval, transportation, and processing require these aforementioned sludge characteristics. These characteristics have a direct bearing on such determinations as the personnel dose rates during handing, the accountability of Special Nuclear Materials, and the acceptability of K Basin sludge to the expected disposal path--the Tank Waste Remediation System. 
WHC-SD-SNF-TI-038, Rev. 0

This page intentionally left blank. 
WHC-SD-SNF-TI-038, Rev. 0

\subsection{ANALYSES AND RESULTS}

Calculations based on measured data were made for both total mass and spatial mass distributions for $K$ East Basin floor sludge constituents, i.e., elements, isotopes. Additionally, in the process of calculating the spatial distribution of the constituents, concentration distributions were generated as an intermediate step, and these are also provided.

\subsection{ANALYSIS OF SLUDGE CONSTITUENTS CONCENTRATIONS}

These calculations were made by first performing spatial interpolation (surface mapping) of the concentration data given for the measured chemistry of as-settled siudge, as given in Makenas 1996, Appendix C. The interpolation algorithm used (because of the gradients in the sludge surface and the sparsity of data) was the inverse-distance method. These calculations, along with subsequent plotting were done using the TECPLOT scientific data visualization computer code running on a SUN workstation. Here the value of a variable at a data point in a destination $\left({ }_{d}\right)$ zone is calculated as a function of the selected data points in the source $\left({ }_{\mathrm{s}}\right)$ zone. The value at each source data point is weighted by the inverse of the distance between the source data point and the destination data point raised to a power as shown below:

$\varphi_{\mathrm{d}}=\left(\Sigma w_{\mathrm{s}} \varphi_{\mathrm{s}}\right) / \Sigma w_{\mathrm{s}}$ (summed over selected points in the source zone)

where:

$\varphi_{\mathrm{d}}$ and $\varphi_{\mathrm{s}}$ are the values of the variables at the destination and source point respectively, and $w_{s}$ is the weighing function defined as:

$w_{s}=D^{-n}$; where $D$ is the distance between the source point and the destination point, and $\mathrm{n}$ is a exponent chosen as 3.5 , to best fit the data (see Section 4.1).

The source points were chosen as the nearest point in each adjacent octant (unless at a boundary).

The basin was mathematically considered as continuous, i.e., not as three separate bays. This was done because of the sparsity of data points (locations). This approach is considered reasonable, though some error is inherent, as the majority of sampling locations are not near the interior walls, and hence their effect on interpolated values near the wall locations becomes less dominate. Previous interpolations for KE Bas in sludge volume calculations evaluated each bay separately, however, there was considerably more data.

The interpolated data was then used for both subsequent analysis and as the data base for visualization plots. The concentration data was generally analyzed from all available measurement locations (i.e., 13 or less in some cases) in the $\mathrm{K}$ East Main Basin. These were mapped via interpolation to each basin cubical location $(5,092$ points). 
Three-dimensional (3D) color contour plots were then created using TECPLOT. These plots, show the component concentration in either the appropriate unit of $\mu \mathrm{g} / \mathrm{ml}$ or $\mu \mathrm{Ci} / \mathrm{ml}$, and are provided in Appendix $A$. For reference, measurement data points are superimposed on these plots.

This data, along with the previously analyzed sludge depth data (see Appendix B and Baker 1995) which is also stored on a 5,092 cubical location basis, was then processed with the FORTRAN computer code DWAVG (see Appendix D) which calculates a depth (or volume) weighted average concentration, ${ }^{-} C_{d}$.

Here:

$$
{ }^{*} C_{d}=\Sigma\left(c_{i}{ }^{*} d_{i}\right) / \Sigma d_{i}
$$

Where, $d$ is the sludge depth, $c$ the local concentration, and $i$ the index related to the 5092 cubical locations, which the expression is summed over. The resulting depth weighted average concentrations, for the sludge components evaluated, are given in Table 1 .

For non-fuel/fission related elements (see lower part of Table 1) the largest depth-weighted average concentrations of as-settled sludge were calculated for the following:

$\begin{array}{ll}\text { Iron (Fe) } & 67,500 \mu \mathrm{g} / \mathrm{cc} \\ \text { Aluminum (A1) } & 16,670 \mu \mathrm{g} / \mathrm{cC} \\ \text { Sodium (Na) } & 1950 \mu \mathrm{g} / \mathrm{cc} \\ \text { Calcium (Ca) } & 1750 \mu \mathrm{g} / \mathrm{cc}\end{array}$

For iron and aluminum, the maximum concentrations occur near the South Load Out Pit (cubical location 6720). The maximum concentrations for sodium and calcium occur near the North Load Out Pit (location 6750).

Fuel related isotopes (e.g., fission products), with the exception of cobalt-60, have their maximum concentrations located near cubical 6070, where a localized peak sludge depth of approximately $18 \mathrm{~cm}$ is found. Maximum activity depth-weighted average concentrations of as-settled sludge were calculated for the following:
Cesium-137 ( $\left.{ }^{137} \mathrm{Cs}\right)$
Strontium-90 $\left({ }^{90} \mathrm{Sr}\right)$
$190 \mu \mathrm{Ci} / \mathrm{Cc}$
$179 \mu \mathrm{Ci} / \mathrm{CC}$

Maximum gravimetric depth-weighted average concentrations of these isotopes were calculated for the following:

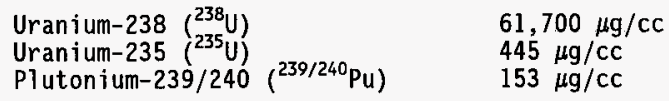




\section{Table 1. K East Basin Floor Sludge Constituents: Calculated Masses and Related Parameters.}

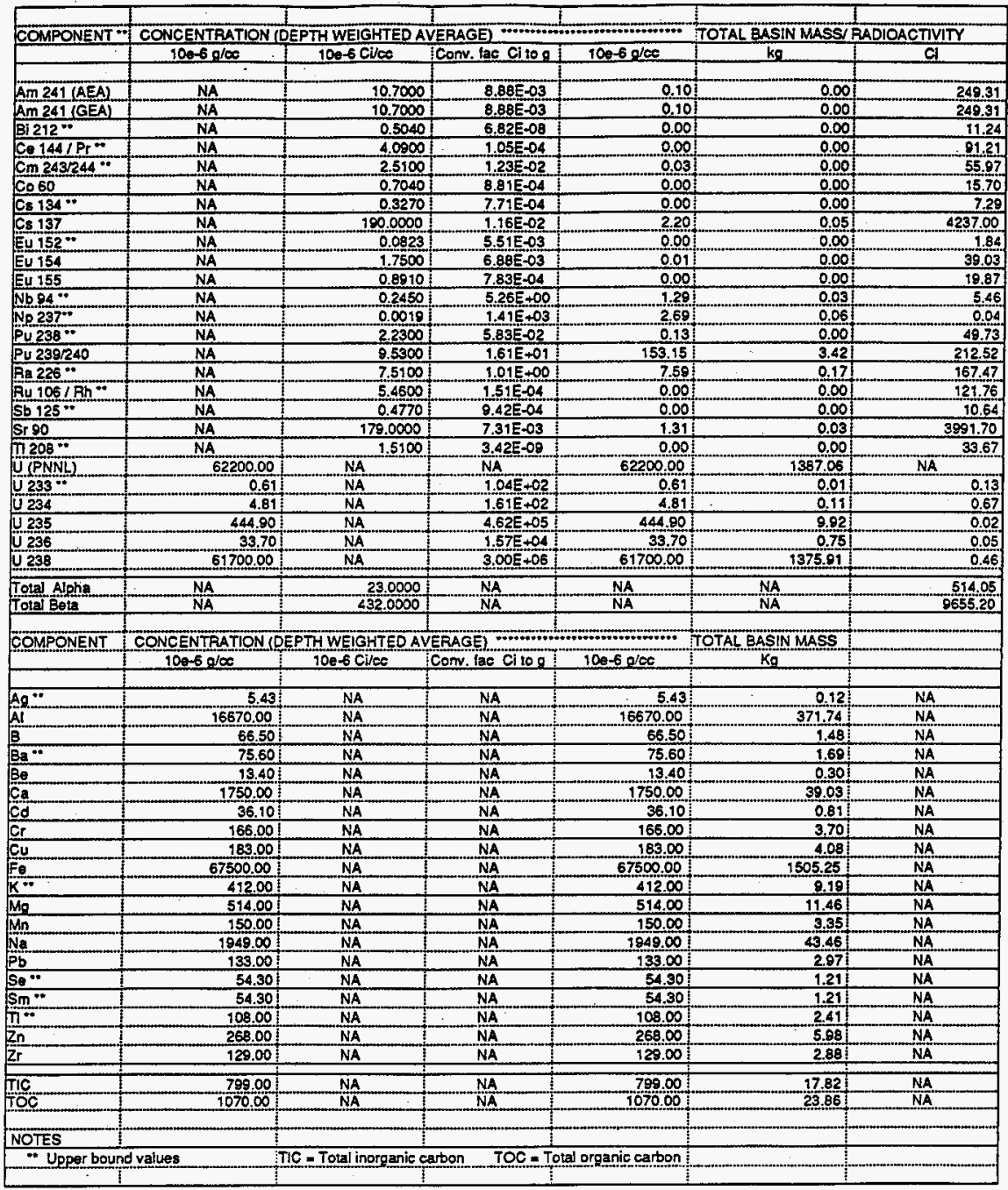


WHC-SD-SNF-TI-038, Rev. 0

\subsection{ANALYSIS OF SLUDGE CONSTITUENTS MASSES}

To determine the total mass of a particular component, the depth weighted average in mass/unit volume is multiplied by the average depth and the effective basin floor area (total basin area minus the area occupied by canisters). These total basin masses are given in Table 1. For radioisotopes, for which concentrations are given in units of radioactivity/ volume, a conversion to mass/volume was made by multiplying the values by the inverse of the associated specific activity.

Here:

$$
\mathrm{g} / \mathrm{C} \mathrm{j}=(\text { At.Wt. }) *\left(\text { half } 1 \text { ife in sec) } / 1.13 \times 10^{13} \mathrm{sec}-\mathrm{C} i / \mathrm{g}\right.
$$

These conversions are given in Table 1.

To obtain spatial mass distributions, the FORTRAN programs MASDIS and MASDIS2 (see Appendix D) were written. These programs read in the sludge depth and concentration data files (plus conversion factors for radioisotopes), and multiply the depth by the mass concentration at each of 5,092 cubical location (the cubical being taken as calculational points). This value is then multiplied by the average effective floor area (assumes canisters are uniformly distributed taking up floor area within the basin) of a cubical location. MASDIS writes out a mass distribution data file which is read by TECPLOT, for subsequent processing and plotting. These mass distribution plots are provided in Appendix C.

The greatest total masses for the non-fuel related elements in the basin floor sludge were calculated for the following:

Fe at $1505 \mathrm{~kg}$

A1 at $372 \mathrm{~kg}$

$\mathrm{Na}$ at $43 \mathrm{~kg}$

$\mathrm{Ca}$ at $39 \mathrm{~kg}$

For fissionable materials the total masses in the basin floor sludge were calculated as:

$$
\begin{aligned}
& { }^{235} \mathrm{U} \text { at } 9.92 \mathrm{~kg} \\
& { }^{239} \mathrm{Pu} \text { at } 3.42 \mathrm{~kg} \\
& { }^{233} \mathrm{U} \text { at } 0.01 \mathrm{~kg}
\end{aligned}
$$

Total basin floor sludge mass calculated for other isotopes of significant amount or interest are as follows:

$$
\begin{aligned}
& { }^{238} \mathrm{U} \text { at } 1376 \mathrm{~kg} \text { (total } \mathrm{U} \text { is } 1387 \mathrm{~kg} \text { ) } \\
& { }^{137} \mathrm{Cs} \text { at } 0.05 \mathrm{~kg} \\
& { }^{90} \mathrm{Sr} \text { at } 0.03 \mathrm{~kg}
\end{aligned}
$$

The calculated distribution of sludge constituent masses showed distinct patterns. Sludge constituent associated with corroded spent fuel were concentrated near the north-west corner of the basin. The north-west corner 
is a region where significant amounts of degraded fuel are located and where the sampling location was between barrels of a canister in a particularly deep area of sludge. Another region of buildup of radionuclides is near the Dummy Elevator Pit. Aluminum and iron, and many other element are mainly located near the mouth of the South Load Out Pit. Significant amounts of Na, Ca, K and $\mathrm{Mg}$ are calculated near the mouth of the North Load Out Pit. A value of $10,200 \mathrm{Ci}$ of alpha + beta radioactivity $\left(\sim 80 \%\right.$ from ${ }^{137} \mathrm{Cs}$ and $\left.{ }^{90} \mathrm{Sr}\right)$ were calculated for the total basin floor sludge, and this was concentrated near the north-west corner of the basin and near the north-east corner near Dummy Elevator Pit. 
WHC-SD-SNF-TI-038, Rev. 0

This page intentionally left blank. 
WHC-SD-SNF-TI-038, Rev. 0

\subsection{DISCUSSION}

\subsection{UNCERTAINTIES}

No attempt was made to statistically determine uncertainties. However, a qualitative discussion is provided here.

The main concern related to uncertainties is the choice of the basin sampling locations for chemical analysis. With only thirteen cubical locations, the choice becomes paramount. Rationale for selection of the sampling locations, based on the current apriori knowledge is discussed in Makenas 1996, and the Sampling Analysis Plan for this effort (Welsh 1994). For example, if the location (6070), where the greatest concentration of spent fuel related isotopes were found, was omitted from the sampling plan, significantly lower amounts of these isotopes would have been calculated by the current analysis.

The choice of interpolation method and the decay exponent in the present analysis affects uncertainties. For example, if the mathematical decay exponent was chosen higher that the 3.5 used, sharper peaks in local concentration and generally lower masses would have been calculated. The converse would lead to the calculation of higher masses. There is not enough data to fully assess this effect. The default TECPLOT exponent of 3.5 , was chosen as it generally fits many natural systems.

The effect of considering the basin as one continuous pool is expected to cause little error. This conclusion is based on the fact that data values near the walls are consistently of low relative concentrations, i.e., not peak values (see Appendix A).

\subsection{COMPARISON WITH PREVIOUS PREDICTIONS}

Previously reported (Smal1 1995; Will is 1995) total basin masses/ activities of sludge constituents were almost universally lower than those reported herein. For the most abundant chemical constituents, previous estimated mass values ranged from between a factor of four (4) to several orders of magnitude lower. For example, the amount of iron was previously report at $315 \mathrm{~kg}$, whereas, this evaluation calculated $1505 \mathrm{~kg}$. For aluminum it was $89 \mathrm{~kg}$ compared to $372 \mathrm{~kg}$.

For fuel/fission products previously reported and (calculated herein) total basin masses were:

$$
\begin{array}{ccc} 
& \text { ld } & \text { New } \\
\underset{\left.{ }_{239} \text { (tota }\right)}{\mathrm{Pu}} & 150 \mathrm{~kg} & (1390 \mathrm{~kg}) \\
& 0.39 \mathrm{~kg} & (3.42 \mathrm{~kg})
\end{array}
$$


For total basin activities:

$$
\begin{array}{lll}
{ }^{137} \mathrm{Cs} & 178 \mathrm{Ci} & (4240 \mathrm{Ci}) \\
{ }^{90} \mathrm{Sr} & 321 \mathrm{Ci} & (3990 \mathrm{Ci})
\end{array}
$$

These totals are consistent with differences in measured concentration values on which they were based, as indicated in the comparisons shown in Table 4.6 of Makenas 1996.

\subsection{GENERAL DISCUSSION}

This analysis provides significant insight on the location and overall mass of constituents in the $K$ East Bas in sludge. The overall amount of materials in the sludge cannot be implied directly from the sample volumes. This follows because the volume of sludge sample taken was adjusted to obtain similar volume samples, even though the sludge depth varied from one location the next on the basin floor (see Makenas 1996).

Several interesting observations were made in the review of the results of this analysis. This analysis clearly shows a concentration of fuel/fission products near the north-west corner of the basin. Furthermore, the evaluation of the constituents shows that the ratio of ${ }^{235} U$ to total $U$ is $0.715 \%$. This may suggest that this material had come from certain models of single pass reactor fuel, which was made from natural uranium $\left(0.714 \%{ }^{235} \mathrm{U}\right.$ content), and not from $\mathrm{N}$ Reactor fuel which was all enriched.

The concentration of $\mathrm{Na}, \mathrm{Ca}, \mathrm{K}$ and $\mathrm{Mg}$ near the North Load Out Pit are indicative of trace elements in hard water (early operations) which likely was captured by the resin of the early ion exchange modules. These modules were know to have leaked in this region. 
WHC-SD-SNF-TI-038, Rev. 0

\subsection{ACKNOWLEDGEMENTS}

The author wishes to thank and acknowledge the following for their contribution in the support of this analysis and report: To Mr. L. D. Bruggeman and Mr. M. J. Packer for their assistance in the processing of data and in generation of plots. To Mr. R. B. Baker for comments and editing of this report. 
WHC-SD-SNF-TI-038, Rev. 0

This page intentionally left blank. 
WHC-SD-SNF-TI-038, Rev. 0

\subsection{REFERENCES}

Baker, R. B., "Summary Status of K Basins Sludge Characterization," WHC-SD-SNF-TI-006, January 1995.

Makenas, B. J., T. L. Welsh, R. B. Baker, D. R. Hansen and G. R. Golcar, "Analysis of Sludge from Hanford K East Basin Floor and Weasel Pit," WHC-SP-1182, Apri1 1996.

Short, S. M. and M. M. Beary, "Spent Nuclear Fuel Project Technical Databook," WHC-SD-SNF-TI-015, Rev. 0, August 1995.

Tecplot, Interactive Data Visualization for Scientist and Engineers, Users Manua 1, Version 6, Amtec Engineering Inc., 1993.

Welsh, T. L. et al., 1995, "Sampling and Analysis Plan for Floor Sludge in the 105-K East Main Basin and Weasel Pits," WHC-SD-SNF-PLN-006, Rev. 0.

Willis, W. L., and A. N. Praga, "105-K Basin Material Design Basis Feed Description for Spent Nuclear Fuel Project Facilities," WHC-SD-SNF-TI-009, August 1995. 
WHC-SD-SNF-TI-038, Rev. 0

This page intentionally left blank. 
WHC-SD-SNF-TI-038, Rev. 0

APPENDIX A

VISUALIZATION PLOTS OF CONCENTRATIONS OF SELECTED CONSTITUENTS FOR K EAST FLOOR SLUDGE

NOTE: The majority of plots are provided in monochrome

form. Color plots are available upon request. 
WHC-SD-SNF-TI-038, Rev. 0

This page intentionally left blank. 
\%

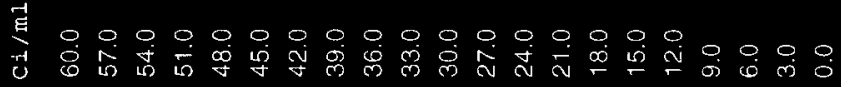

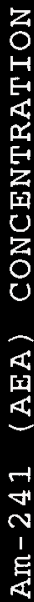

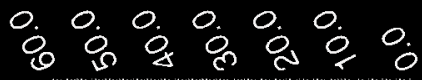

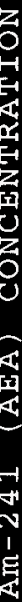

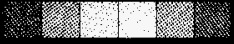

I

[a]

$\underset{⿴}{\varrho}$

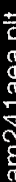

$\frac{1}{0}$

"o

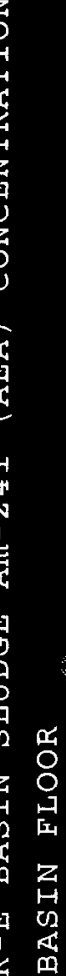

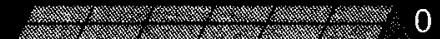

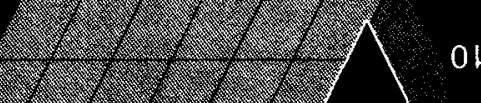

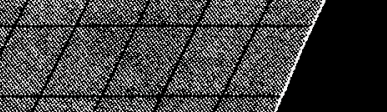

1) - (

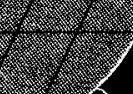

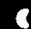

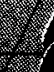

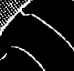

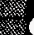

,

6

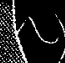

(

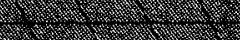




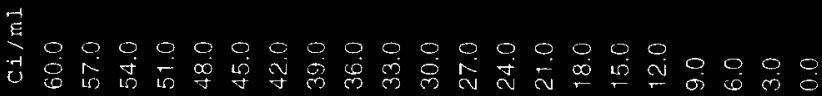

ix

$Z$
0
$H$
$E-1$
14
04
$E$
$z$
'
$U$
$z$
0
0

: $000 \%$

언

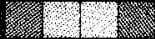

4

委

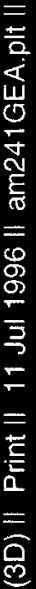

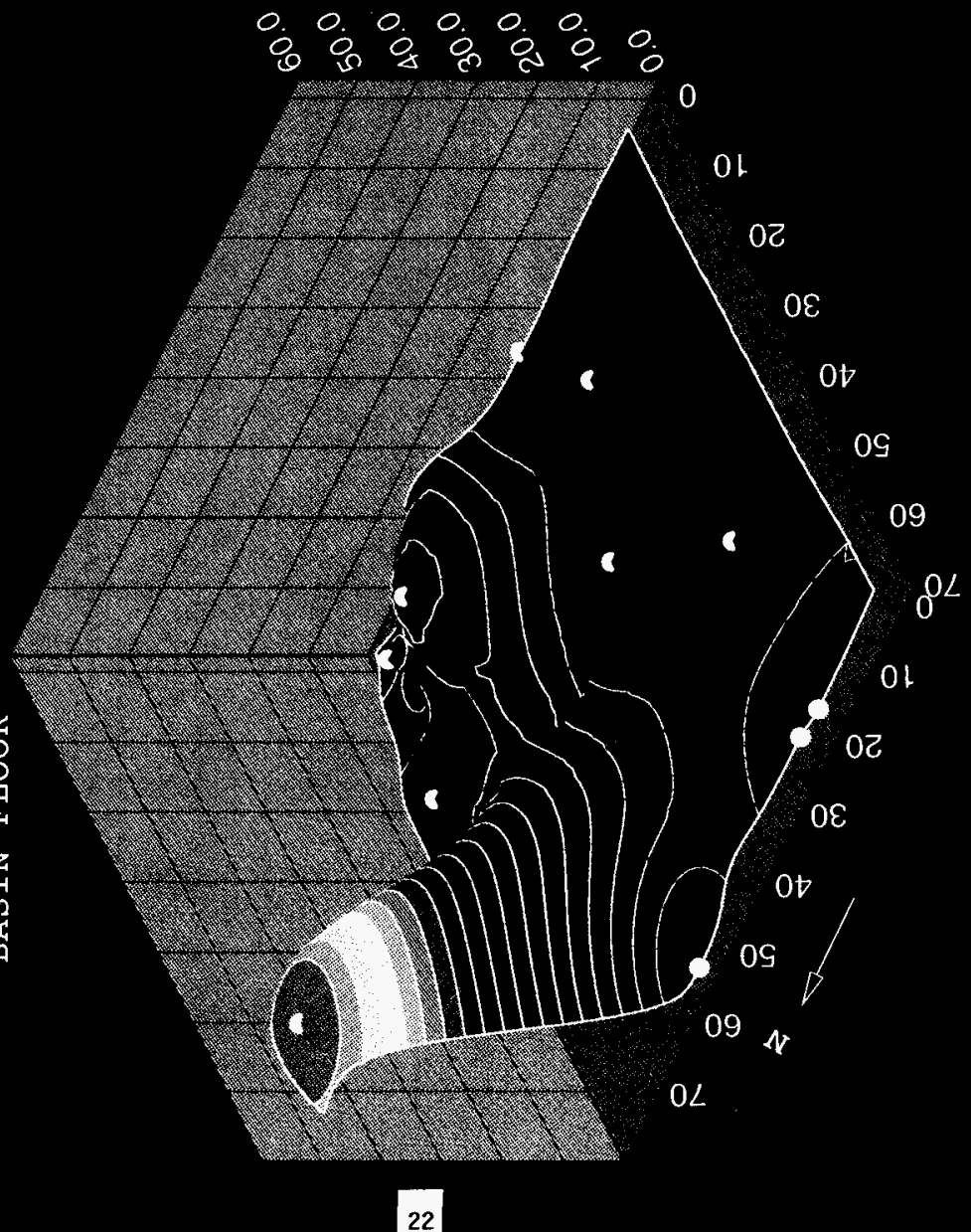




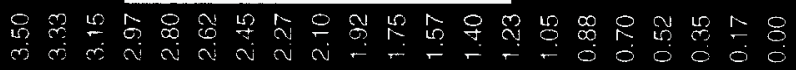
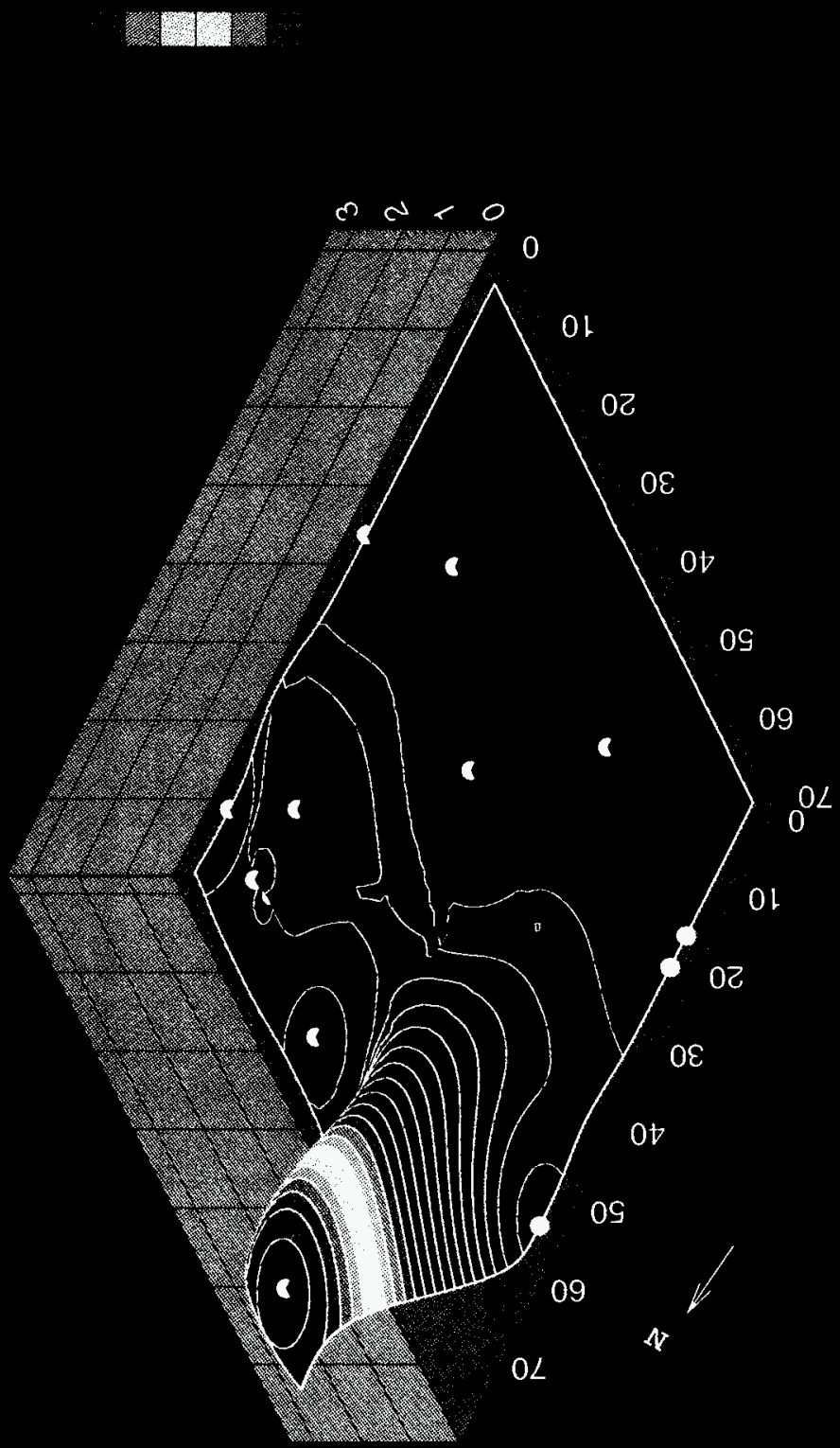


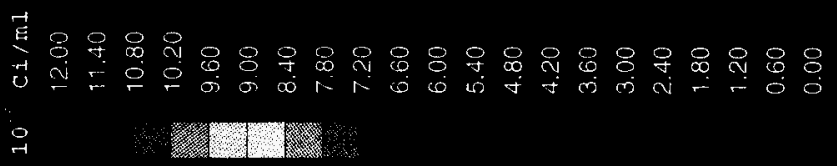

1.
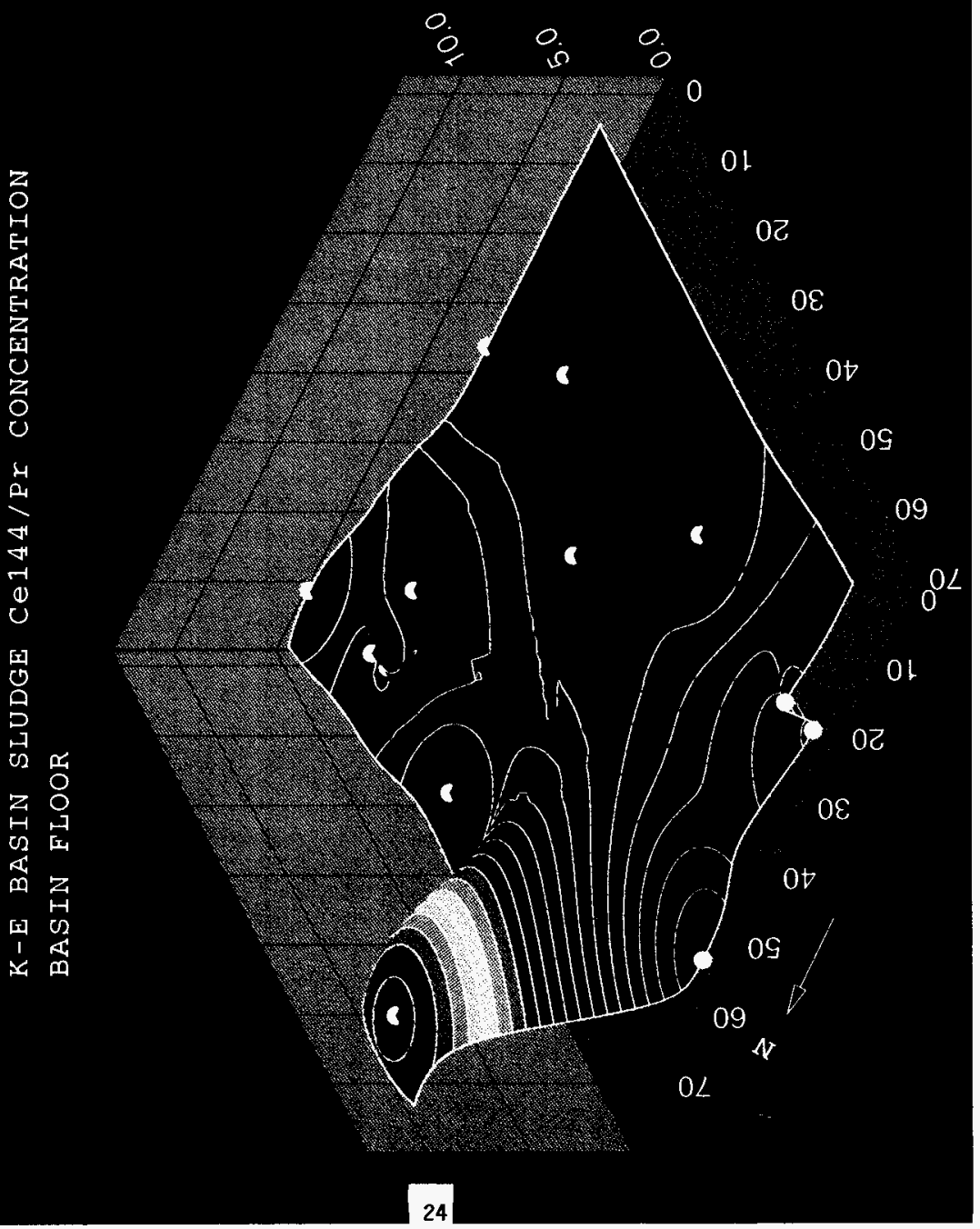

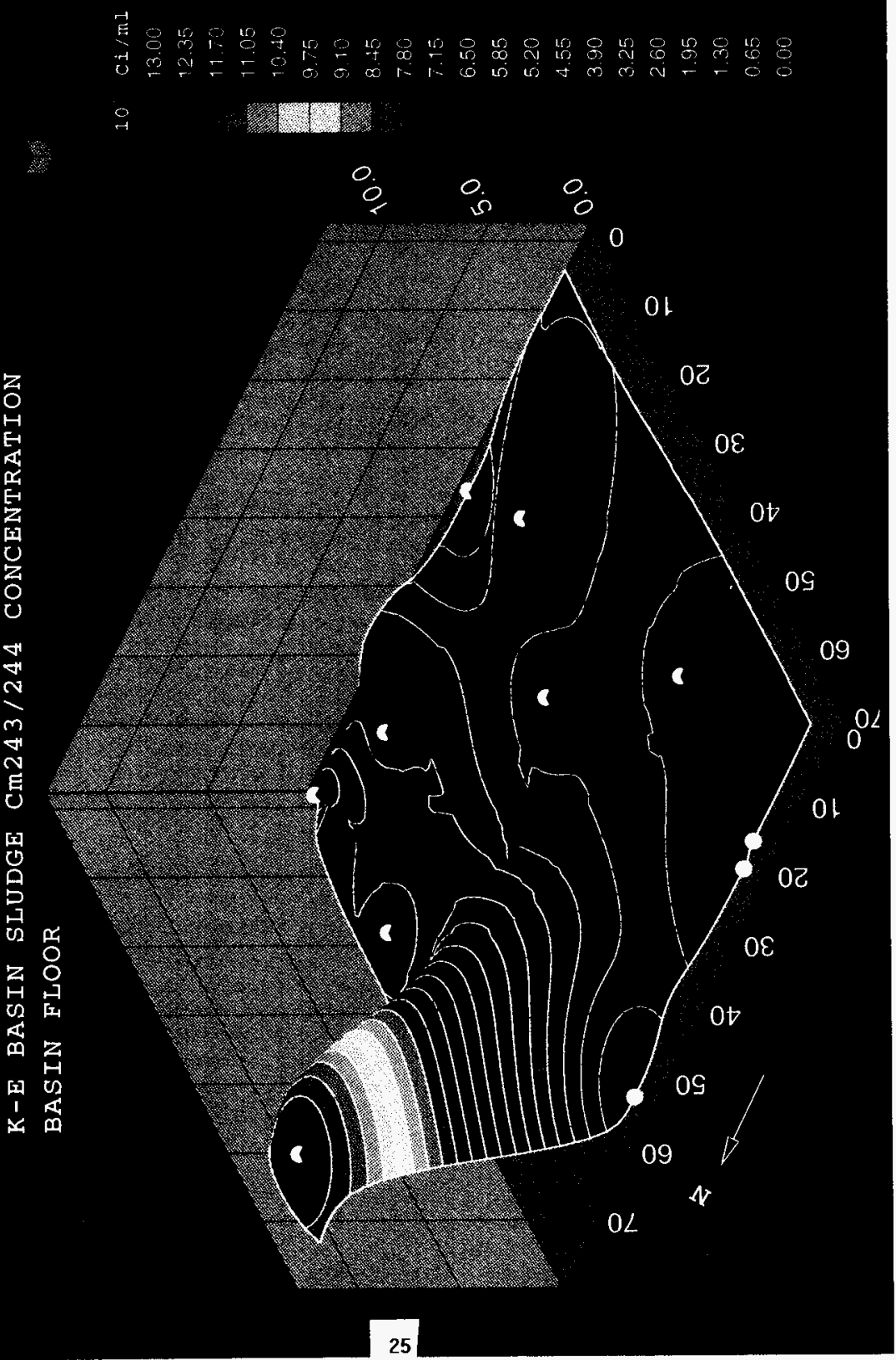
WHC-SD-SNF-TI-038, Rev. 0

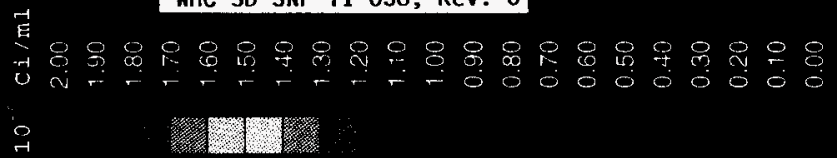

.

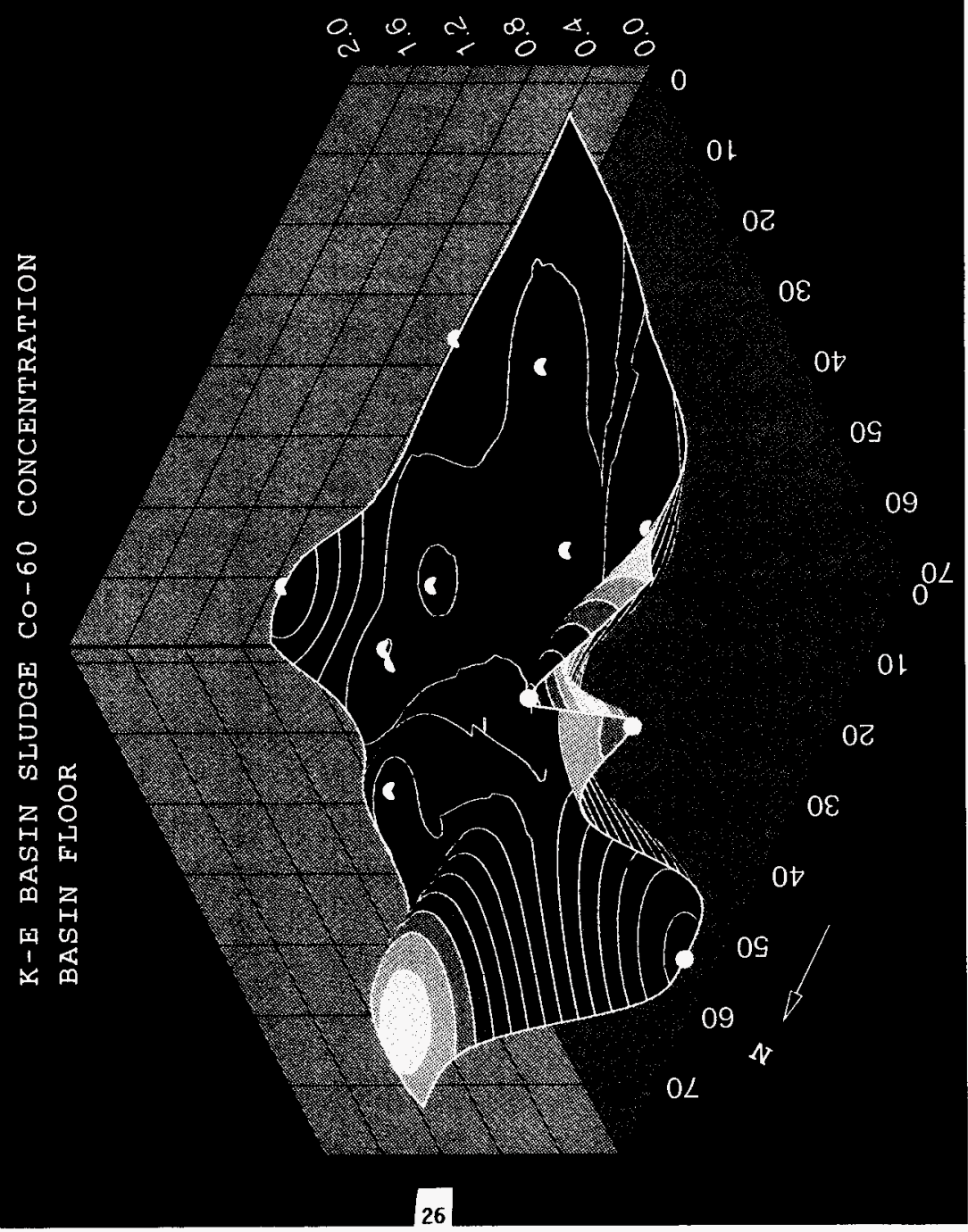




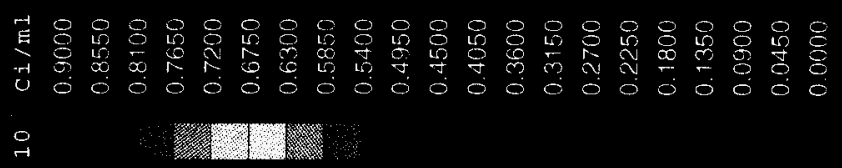

.

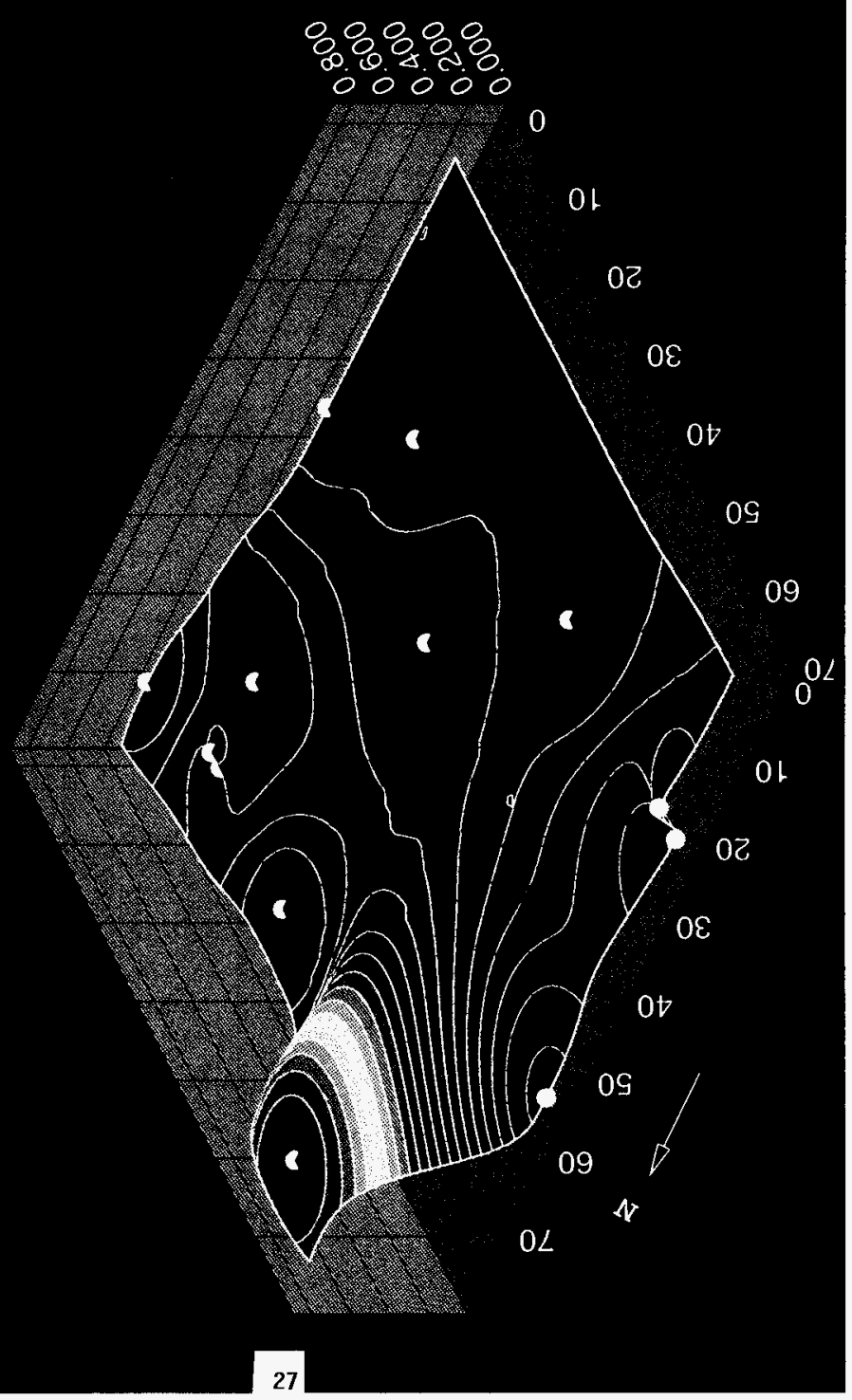


WHC-SD-SNF-TI-038, Rev . 0
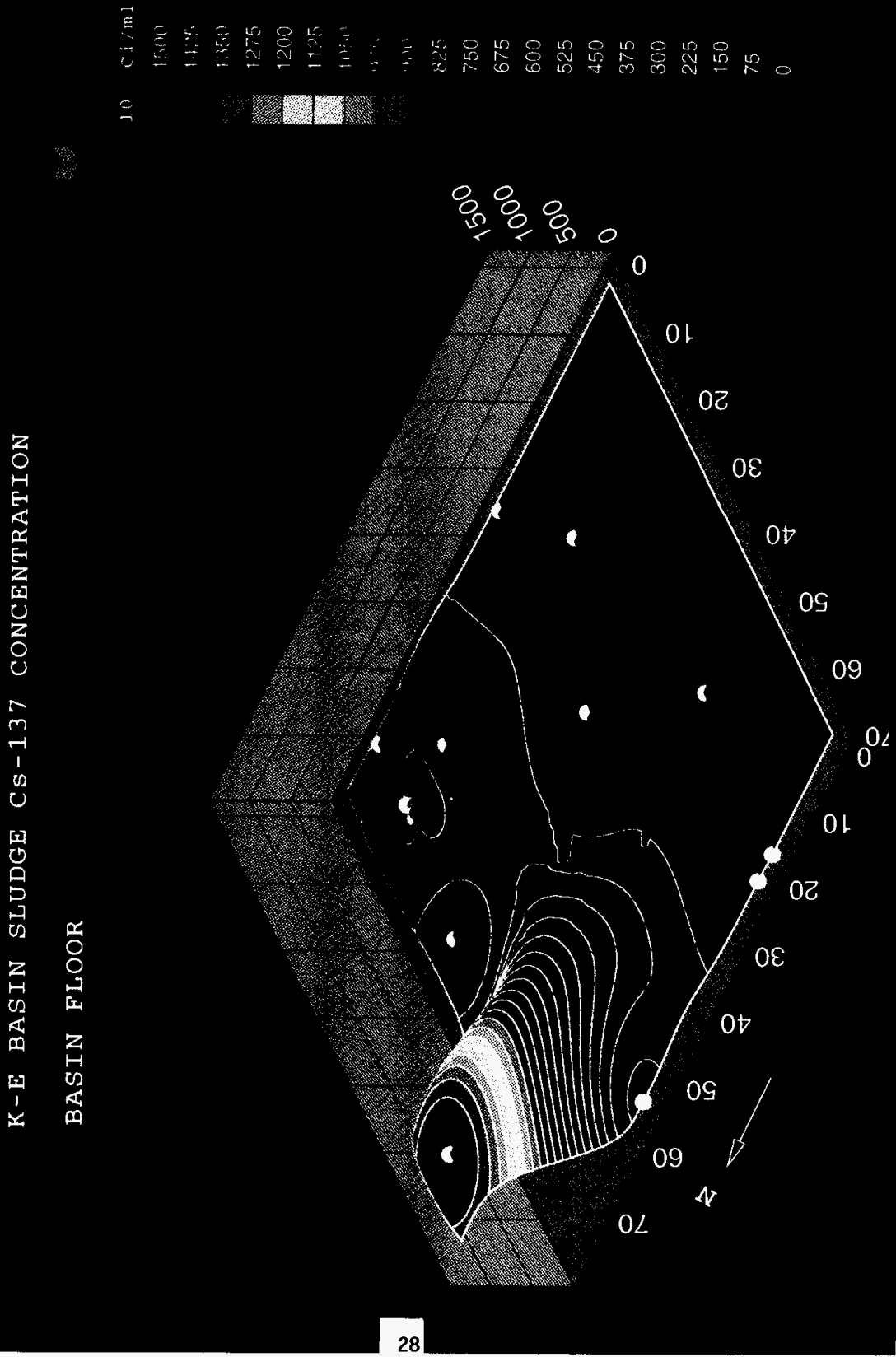
WHC-SD-SNF-TI-038, Rev. 0
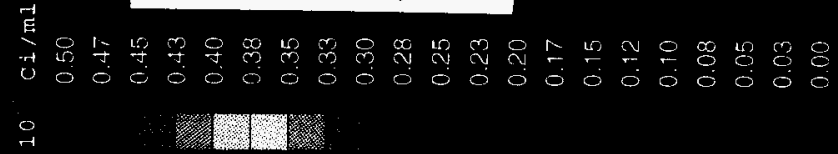

$$
\begin{gathered}
30 \\
: 0.0
\end{gathered}
$$

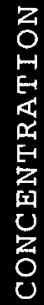

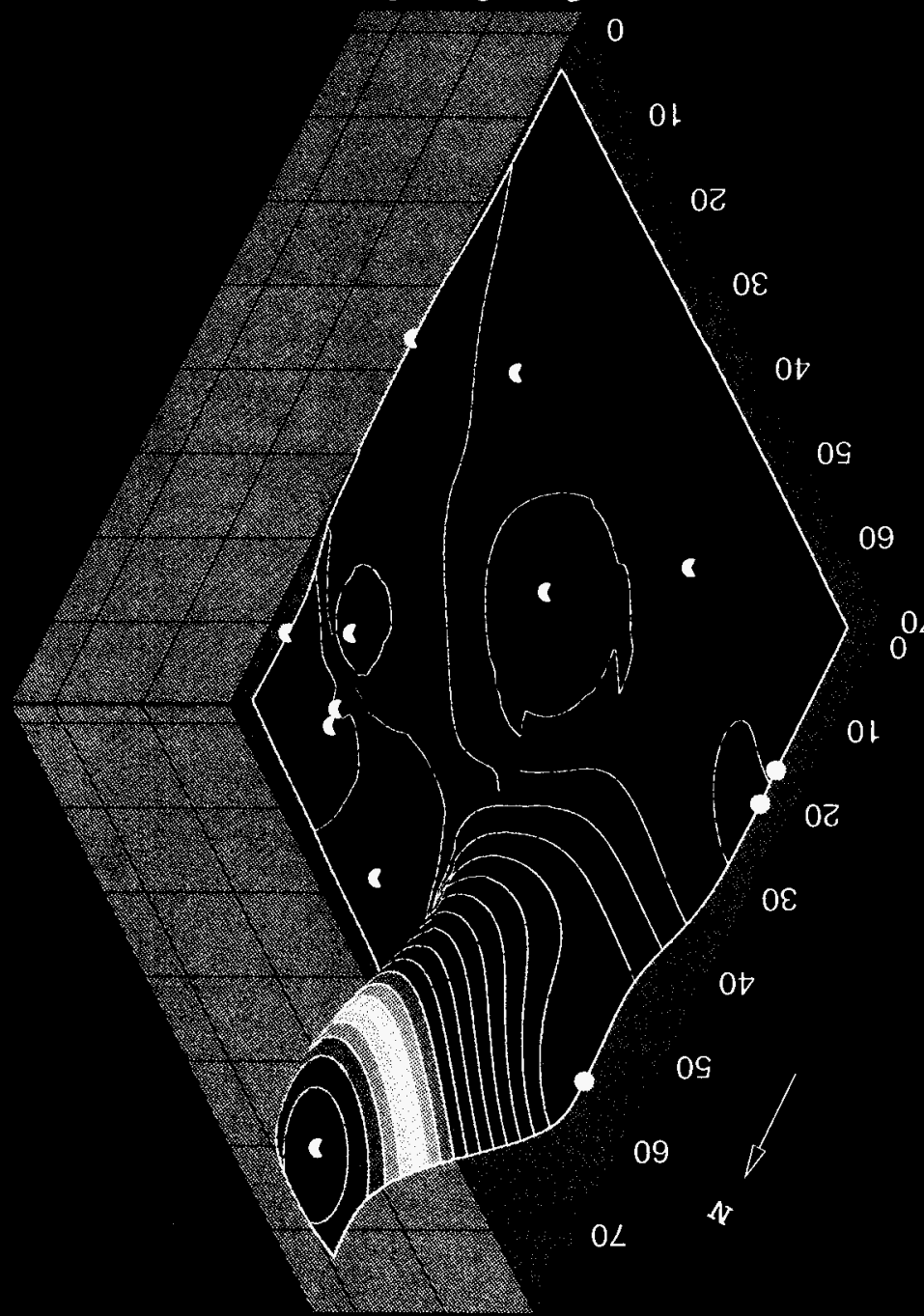




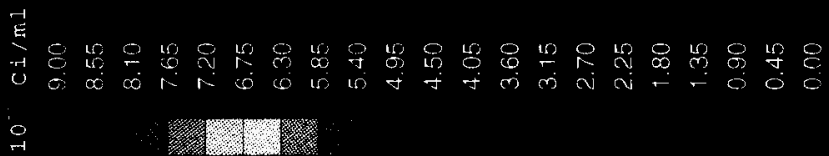

.

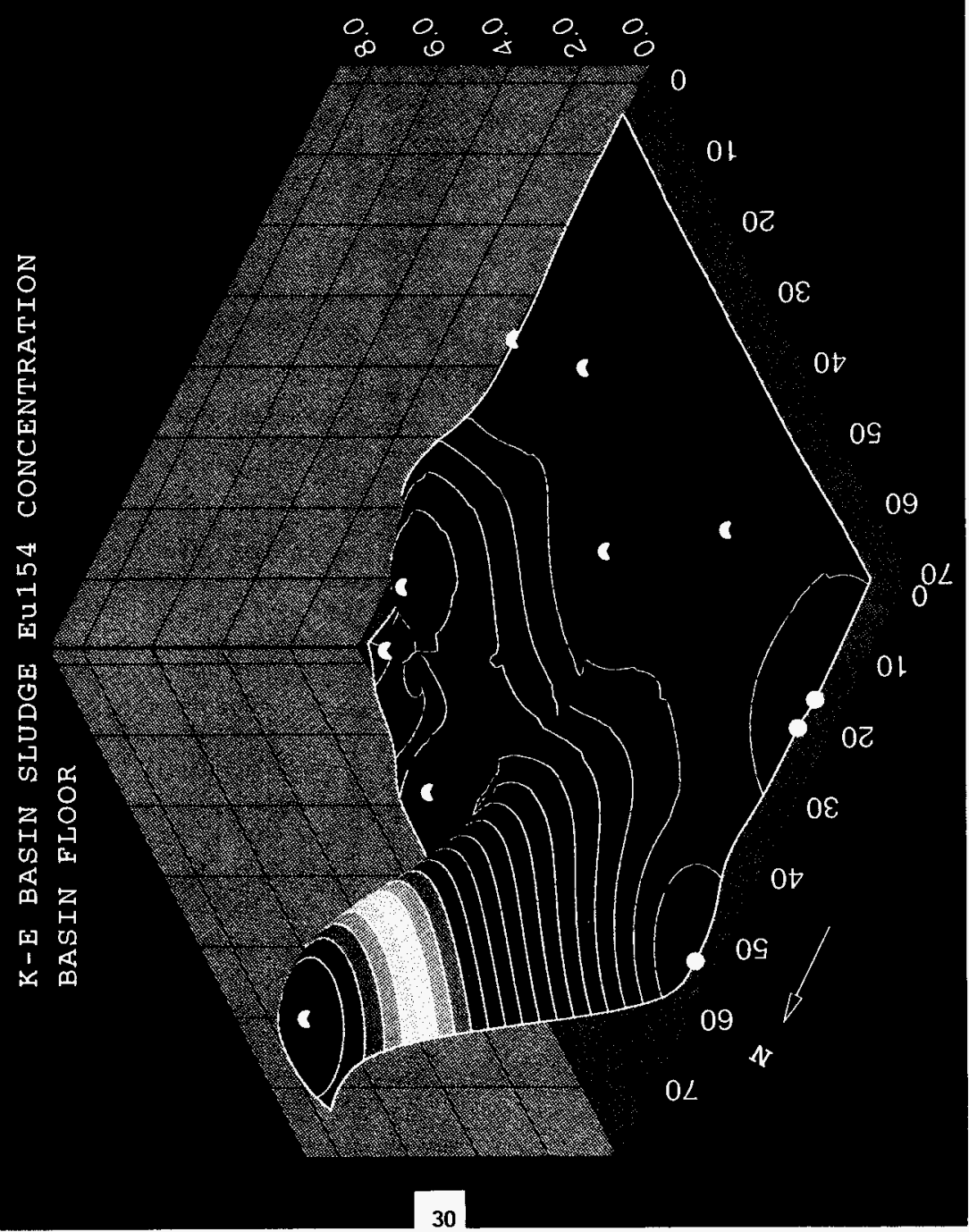


도

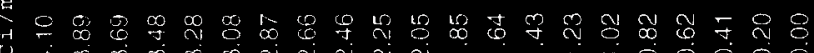

:

.

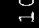

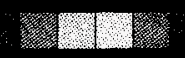

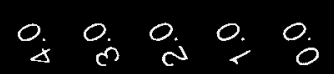

0

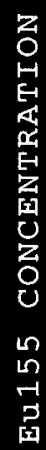

in

n

공

[a]

0

ค

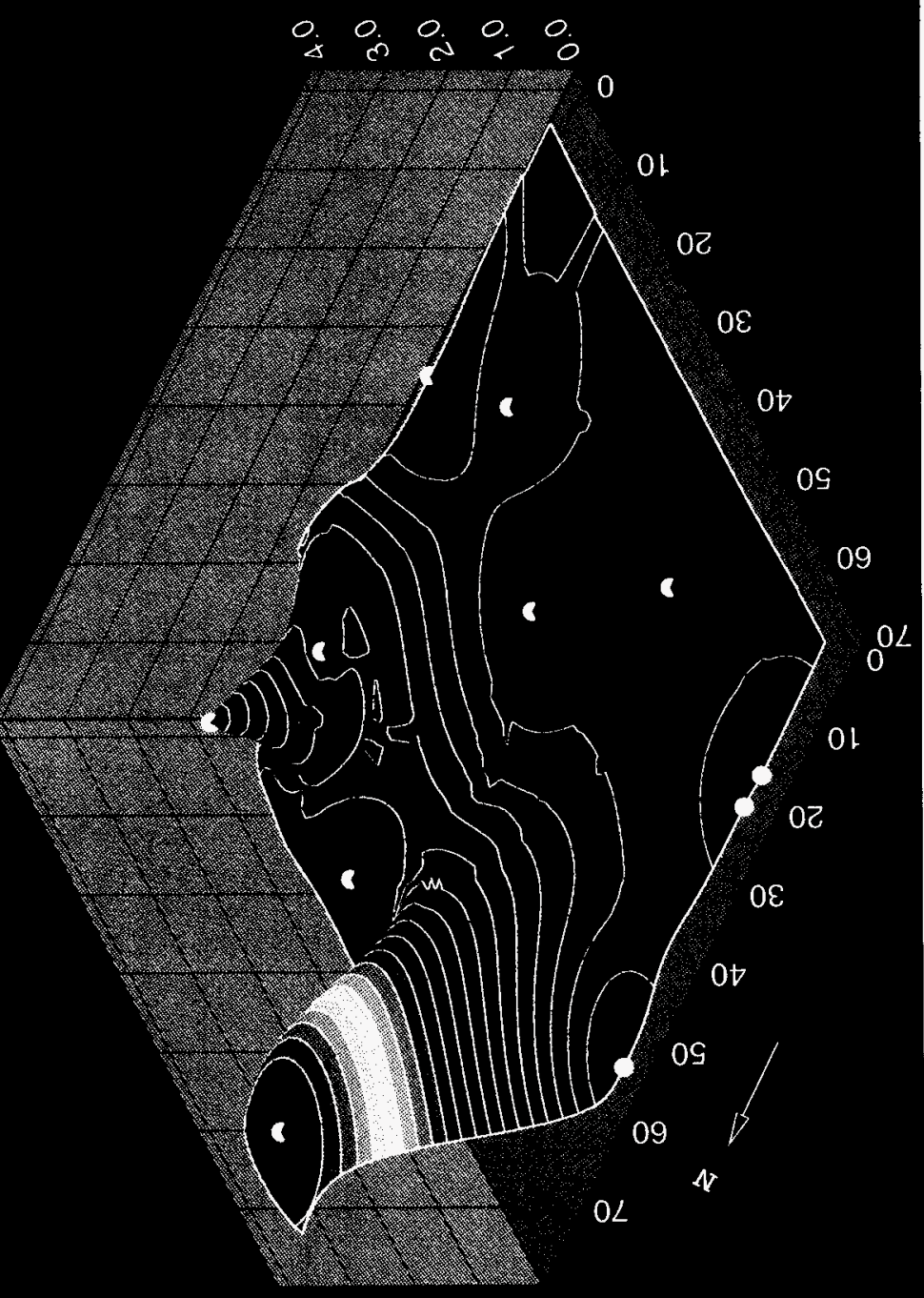


WHC-SD-SNF-TI-038, Rev . 0

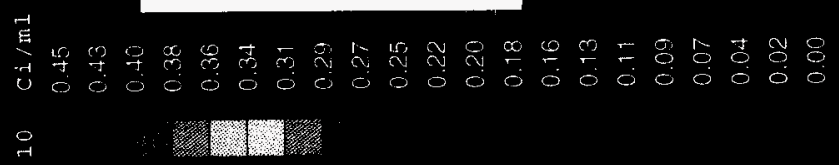

$$
\text { \& } 0 \text { \% : }
$$
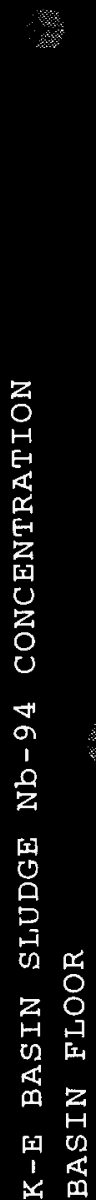

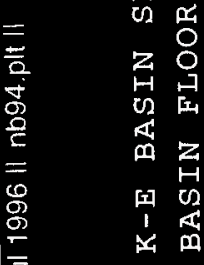




\section{K-E BASIN SLUDGE Np-237 CONCENTRATION}

\section{BASIN FLOOR}

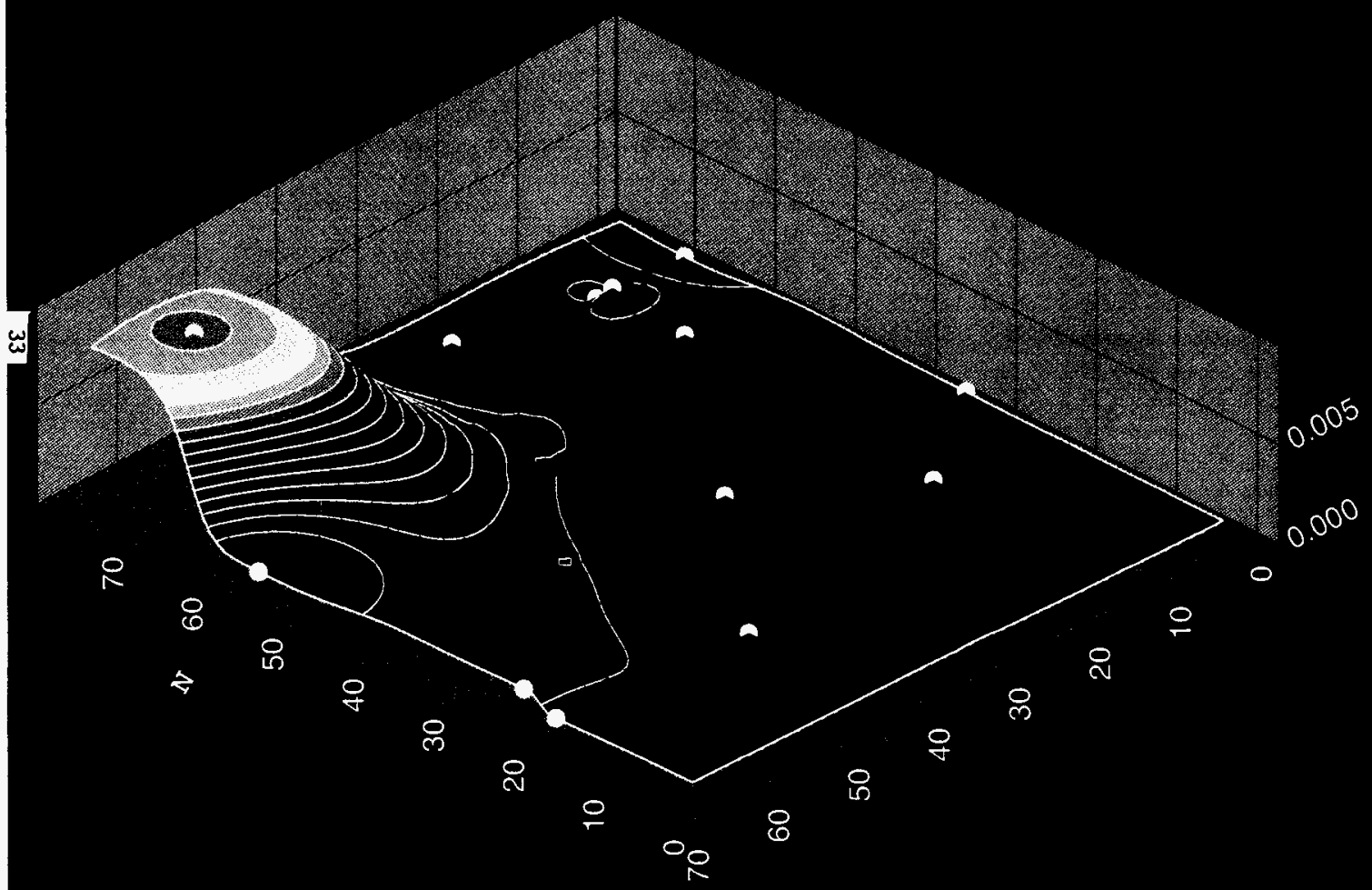




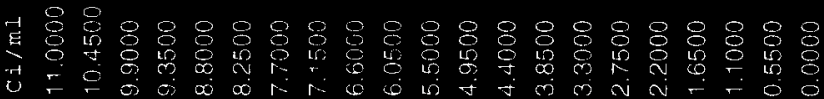

0

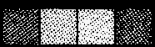

$$
\text { \% } 8 \% \circ \%
$$

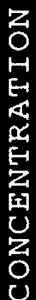

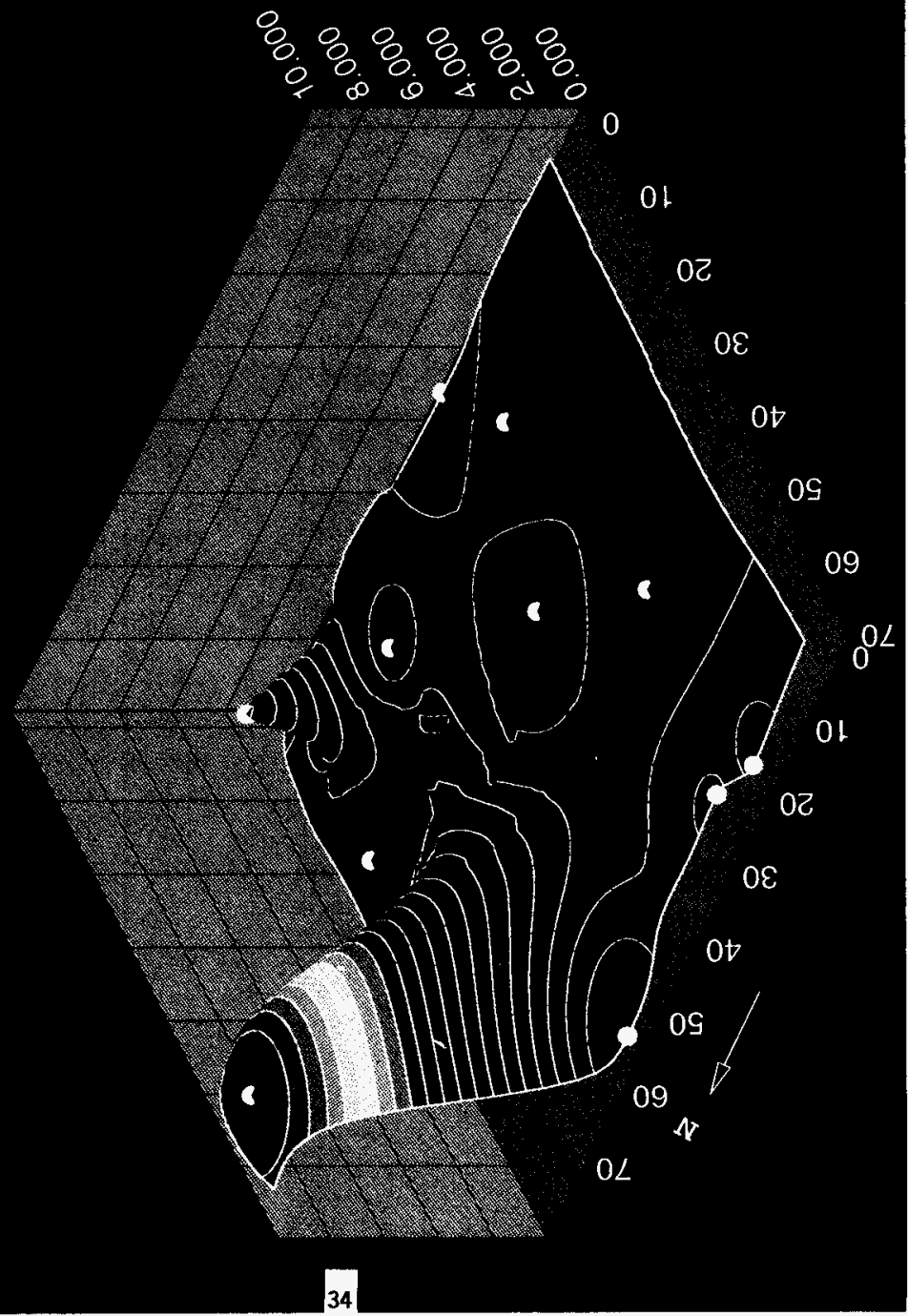

$\infty$

m

i

$?$

모

0

$\equiv \quad$

$\alpha$

$>0$

$\overbrace{1}$

O I I

近

a 1

14

a $m$ 
WHC-SD-SNF-TI-038, Rev. 0

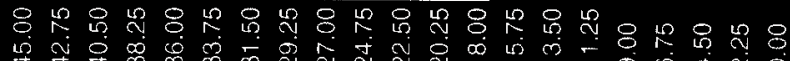

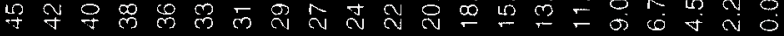
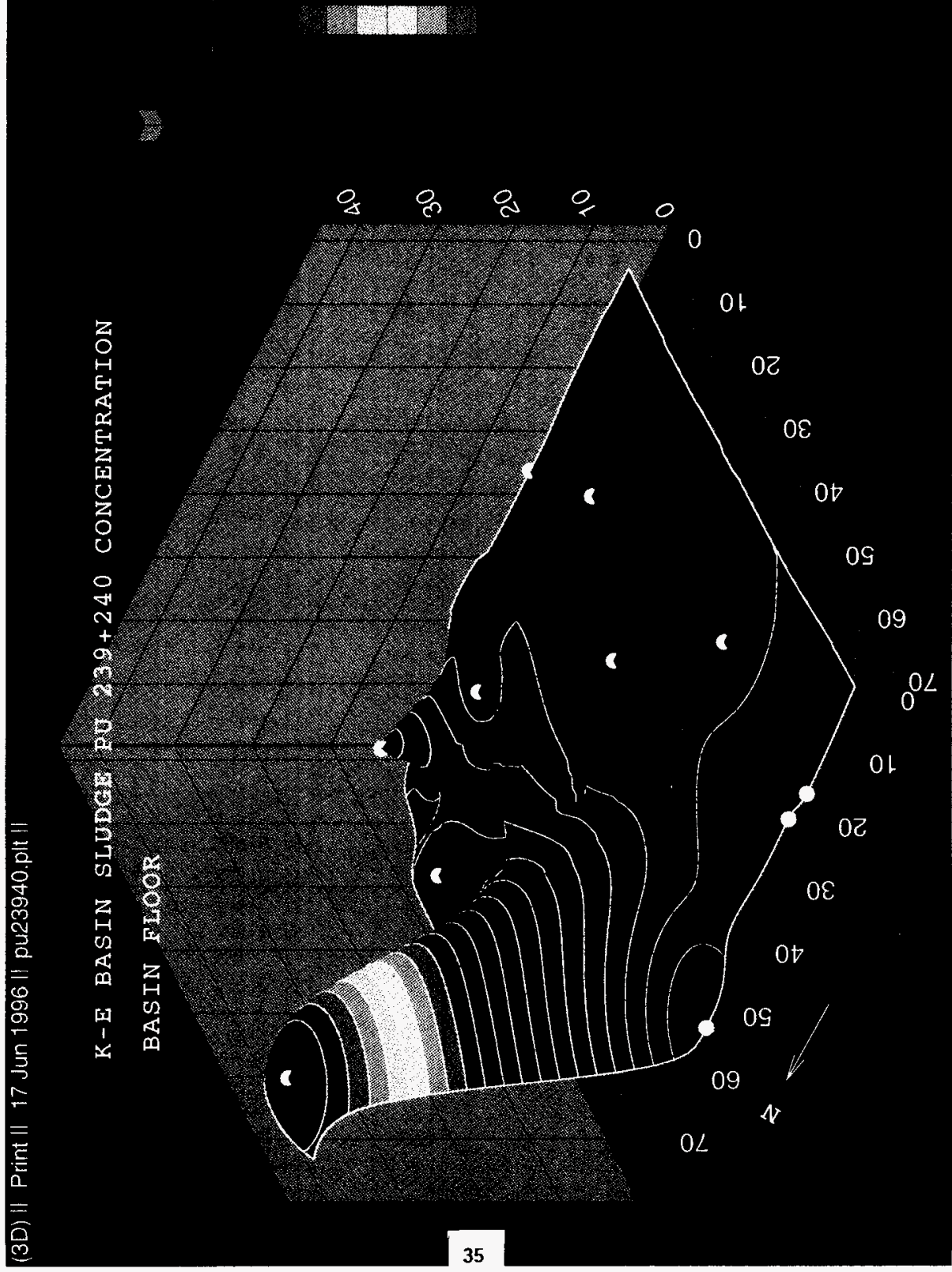
WHC-SD-SNF-TI-038, Rev. 0

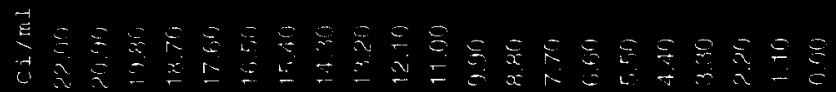

$$
\begin{aligned}
& \therefore \quad \text { ind }
\end{aligned}
$$

$$
\therefore \%
$$
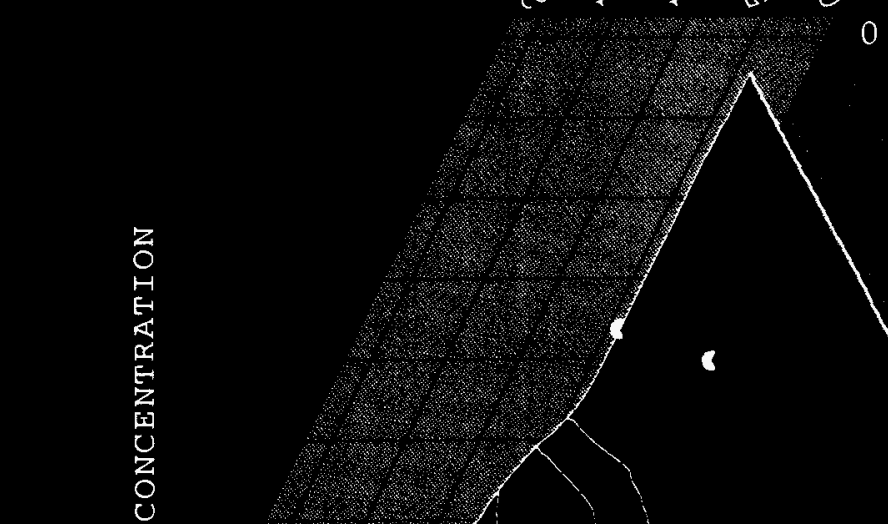

$=$

$z$
0
-1
$E$
$\alpha$
0
$E$
$z$
$z$
$U$
$z$
0
0

$\stackrel{\sim}{\sim}$

N

$\underset{\sim}{\infty}$

뙤

7
0
0
0
0

2 8

$\mapsto$

U2 [ᄃ

需

苗

(19)

$1 \leqslant$

$\approx 0$ 


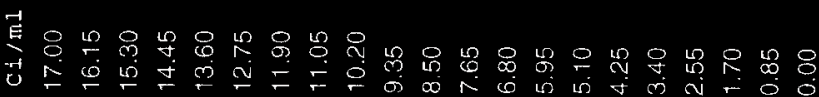

0

.

$$
\text { i } 0 \text { i } 0
$$
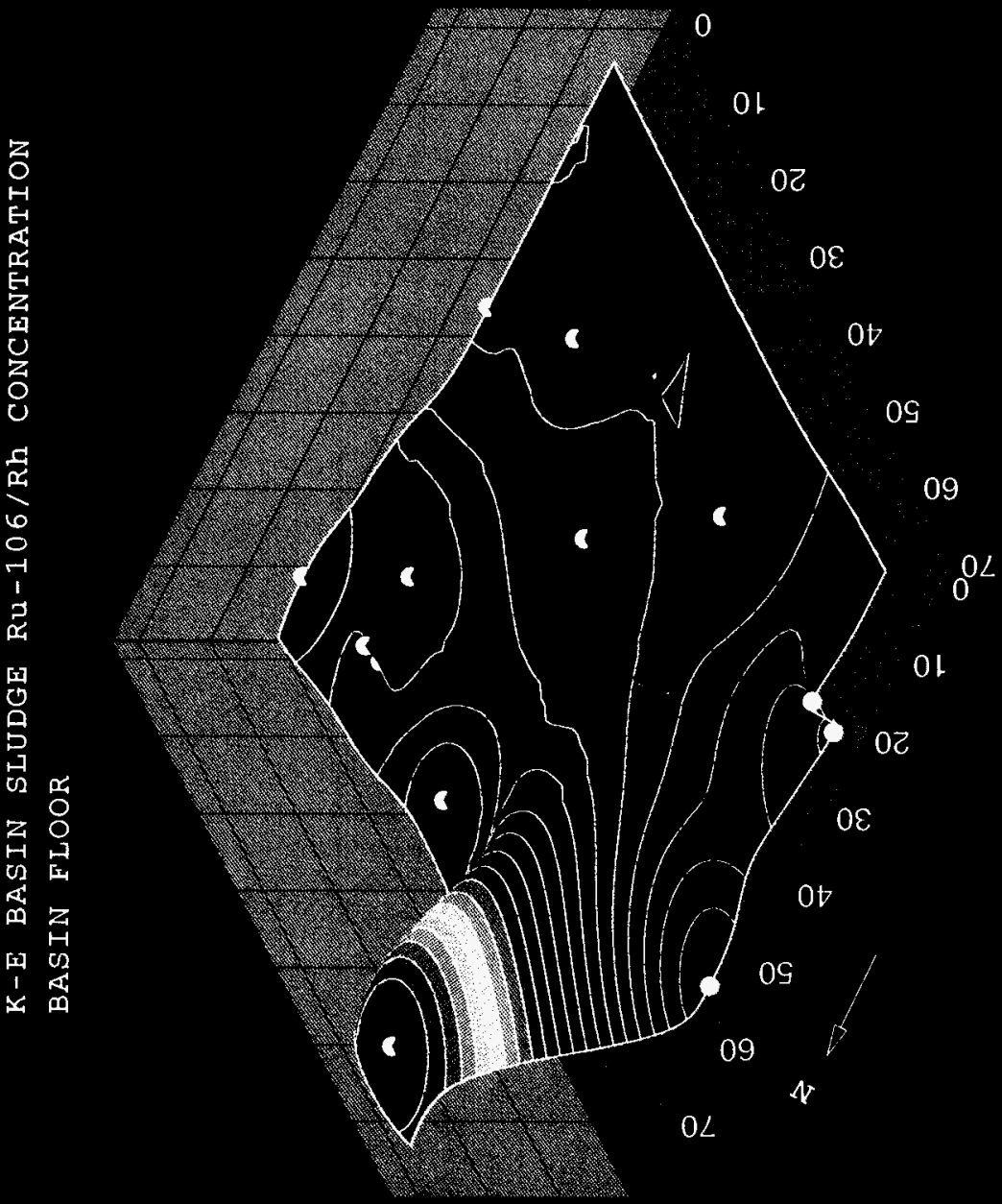


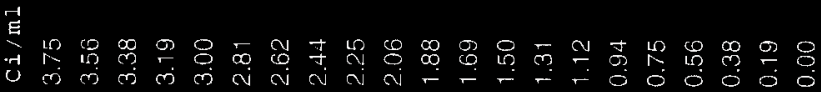

敉

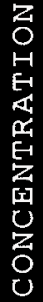

$8_{0} 8^{\circ}, 8_{0}, 8_{0}$

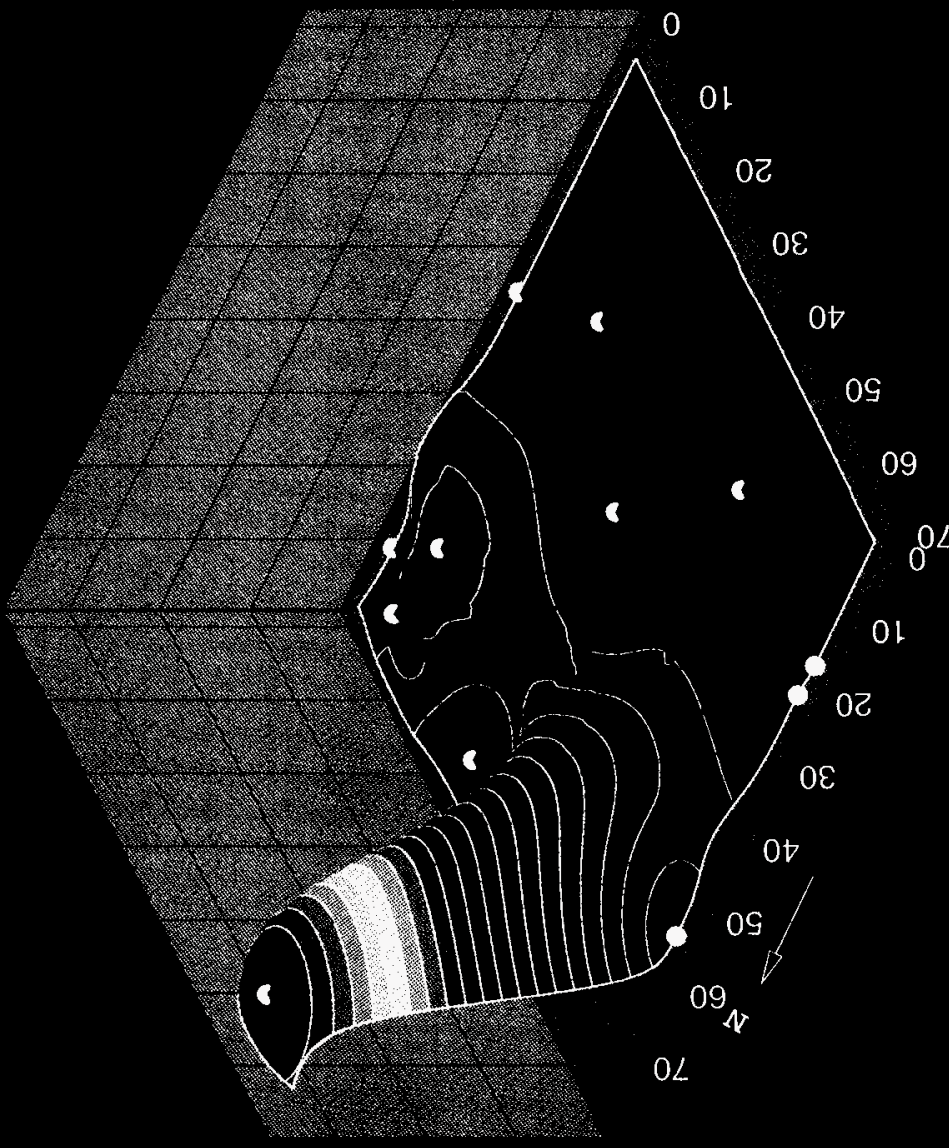

$n$
1
1
0

回

0

回

药

$=$

× $ᄋ$

H

os 応

4

m $\mathrm{Z}$

(9) 0

14

我 
K-E BASIN SLUDGE Sr-90 CONCENTRATION

$\omega$

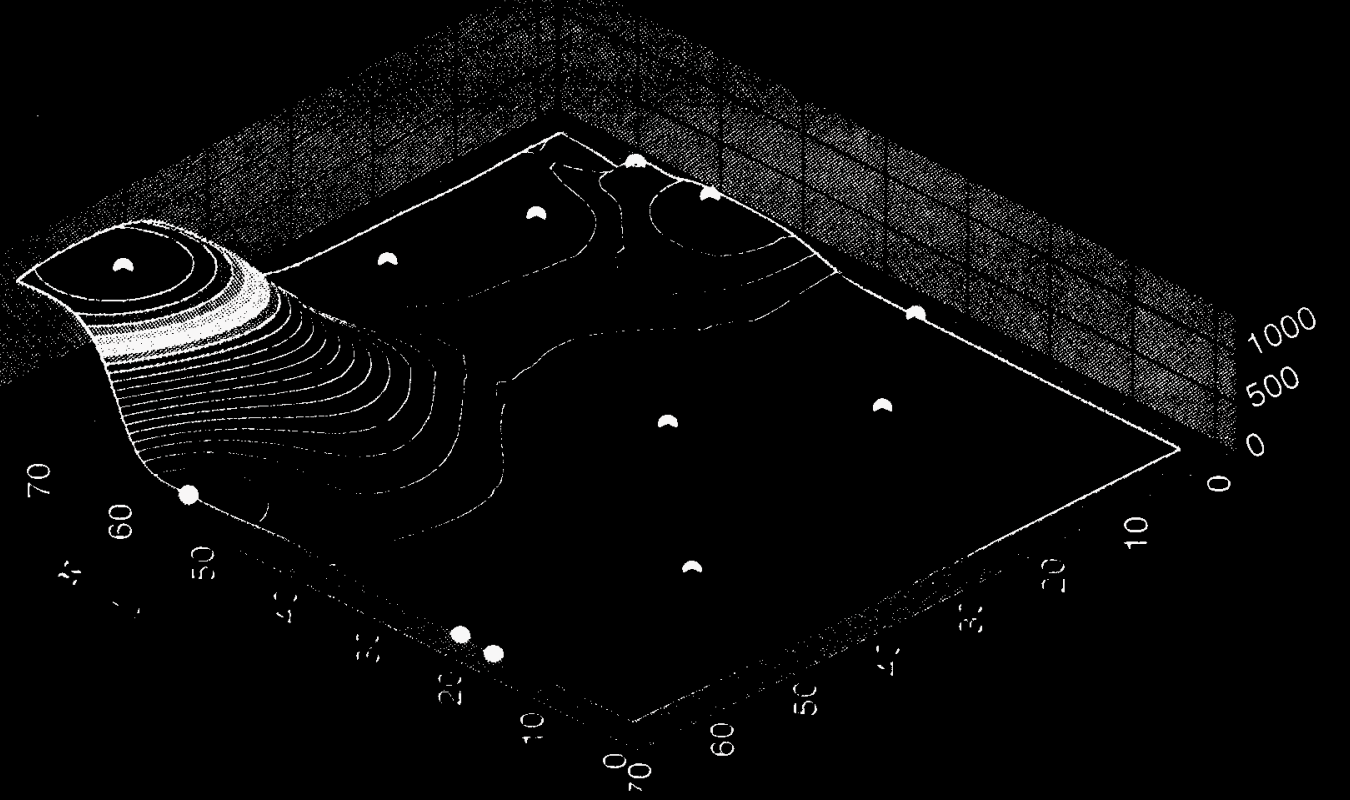


WHC-SD-SNF-TI-038, Rev . 0

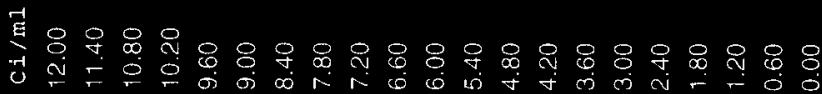

3x

임
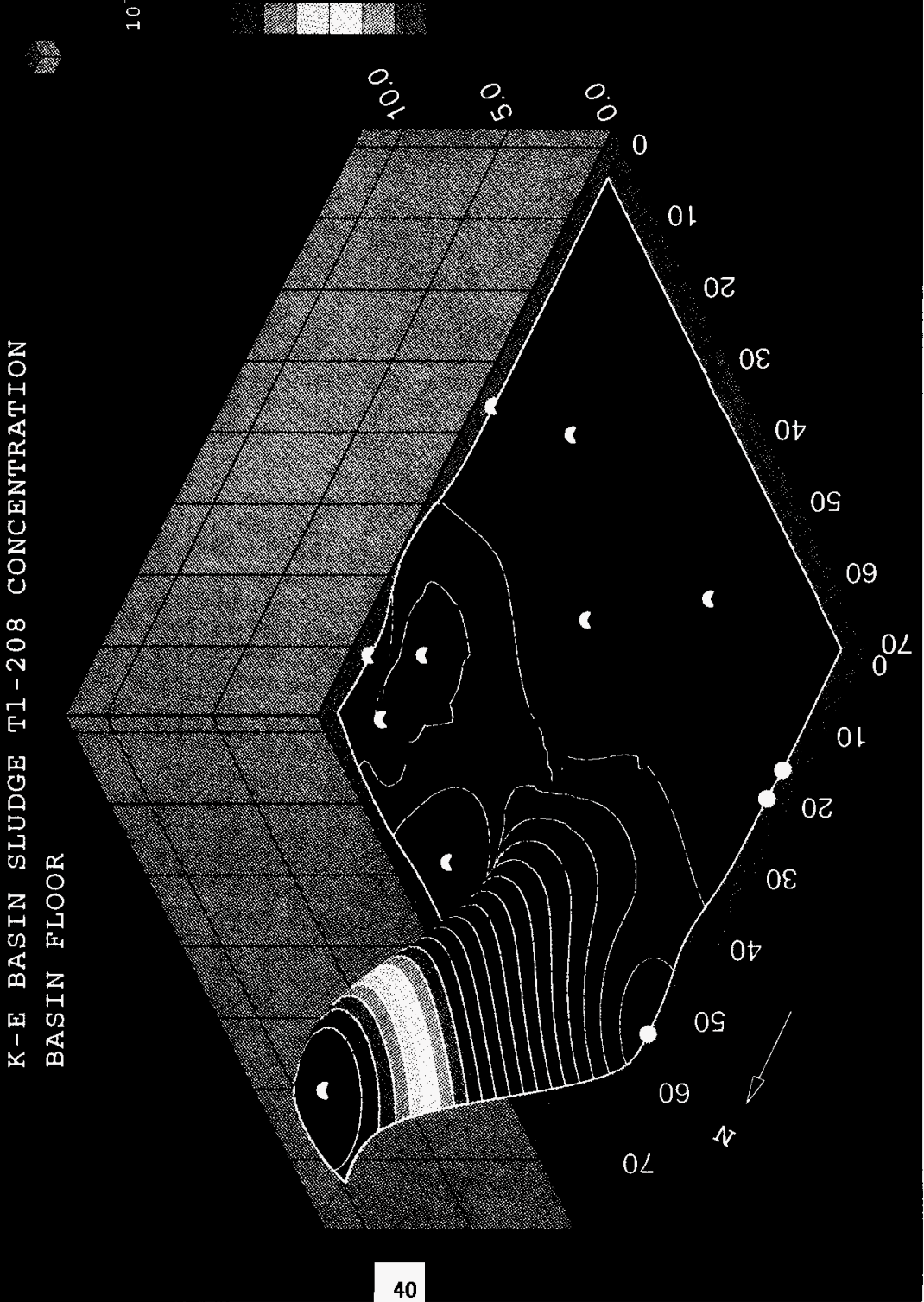


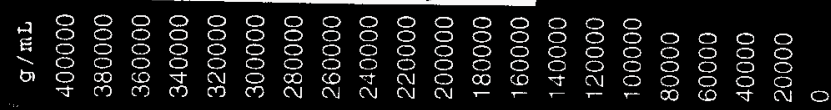
'임

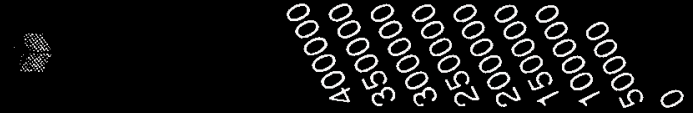

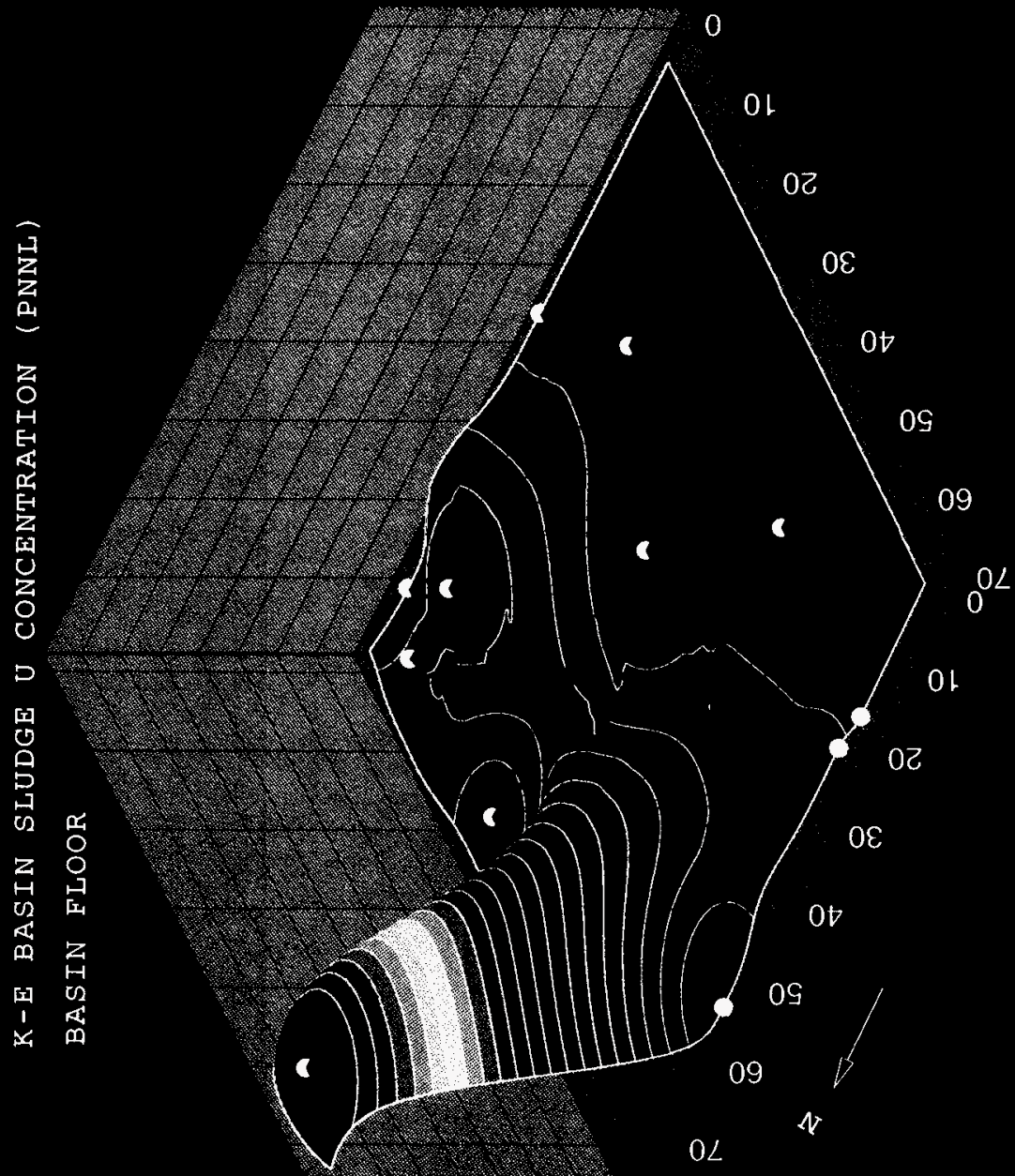




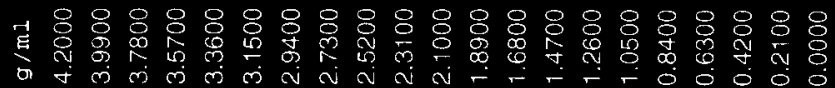

윽

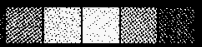

잔
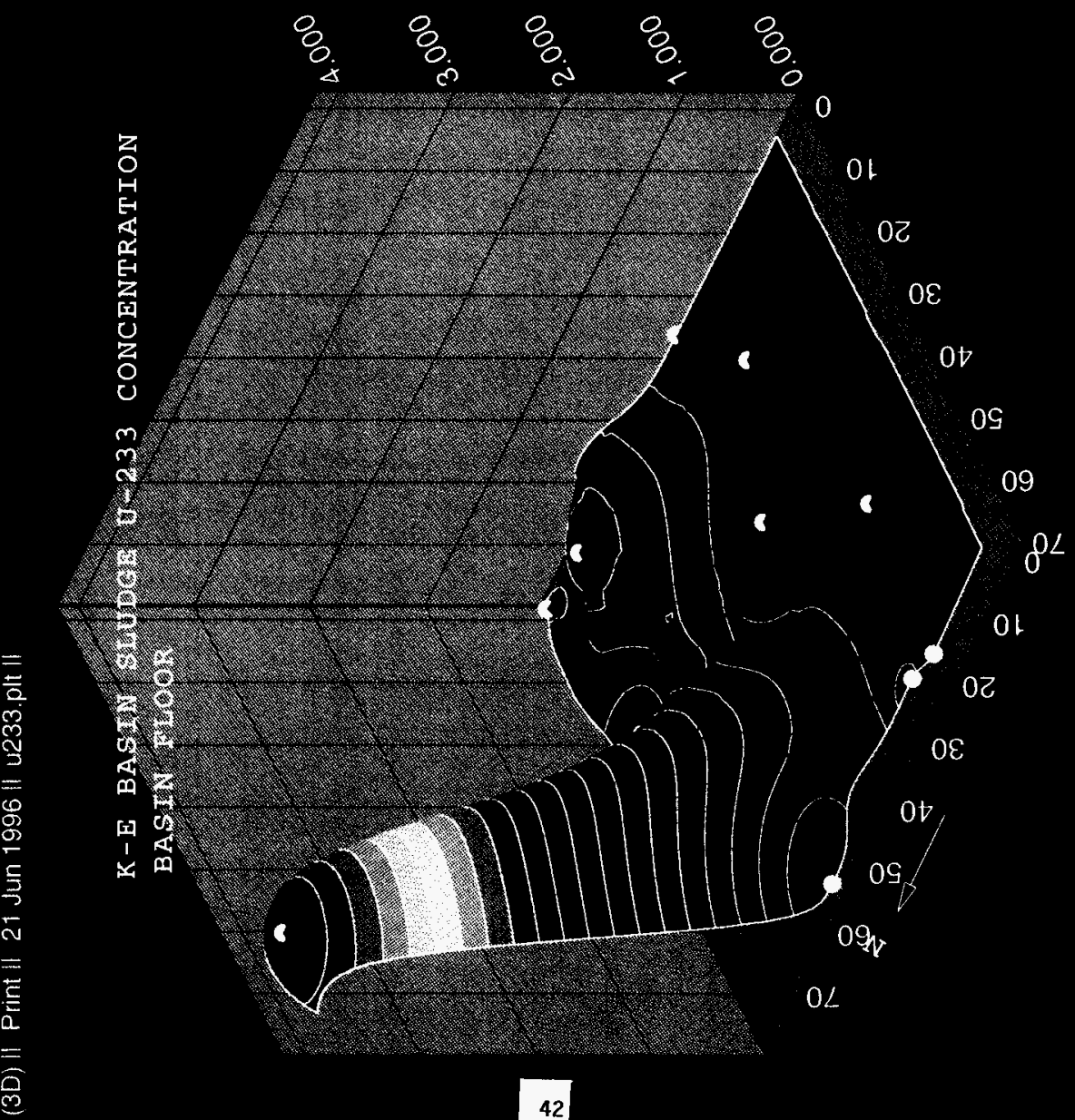


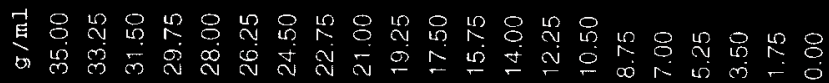

k

0
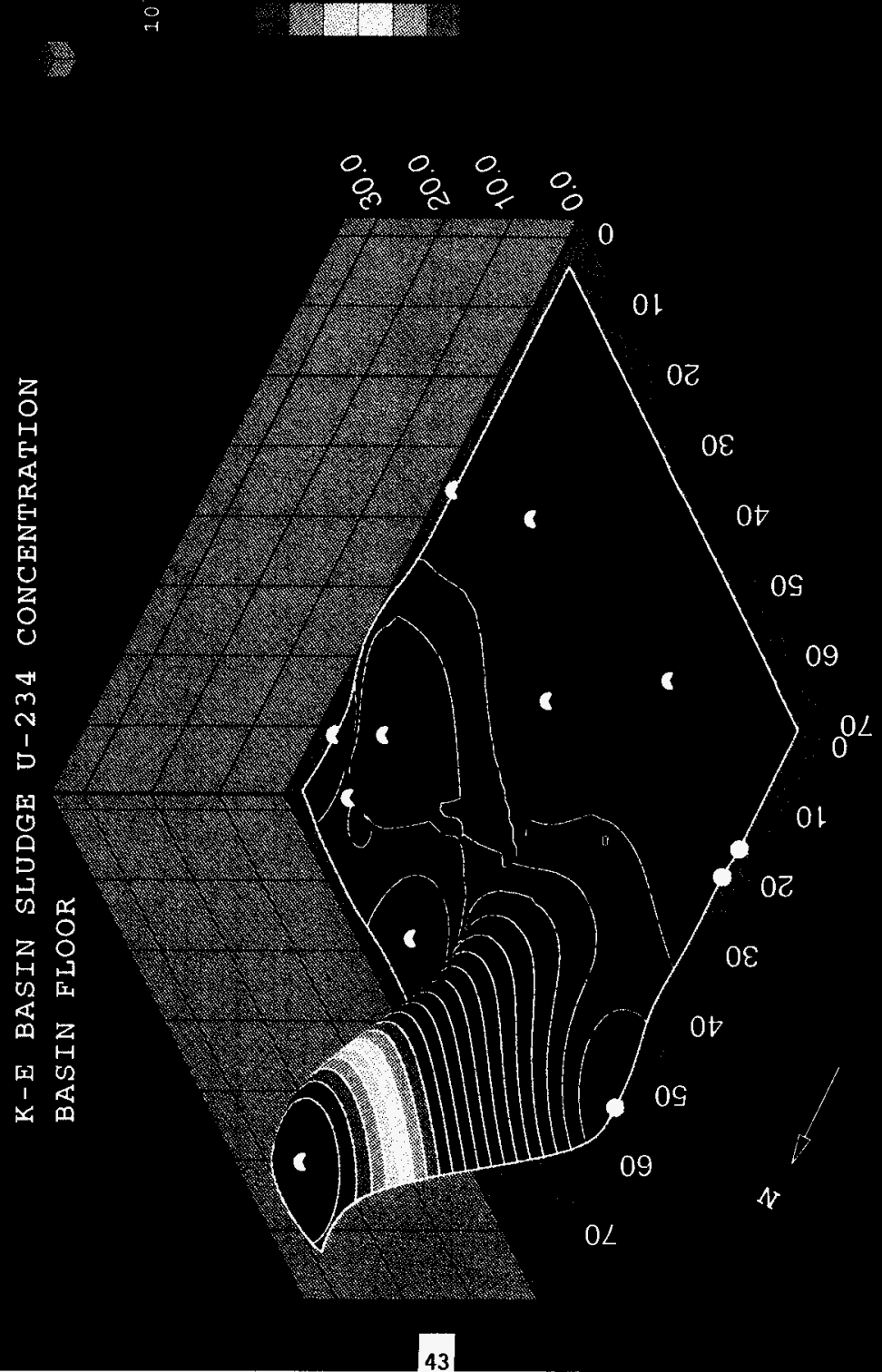
WHC-SD-SNF-TI-038, Rev. 0

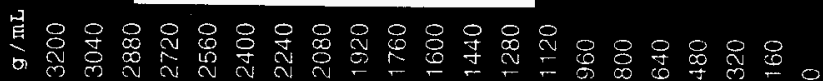

$\stackrel{0}{-1}$

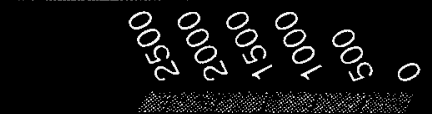

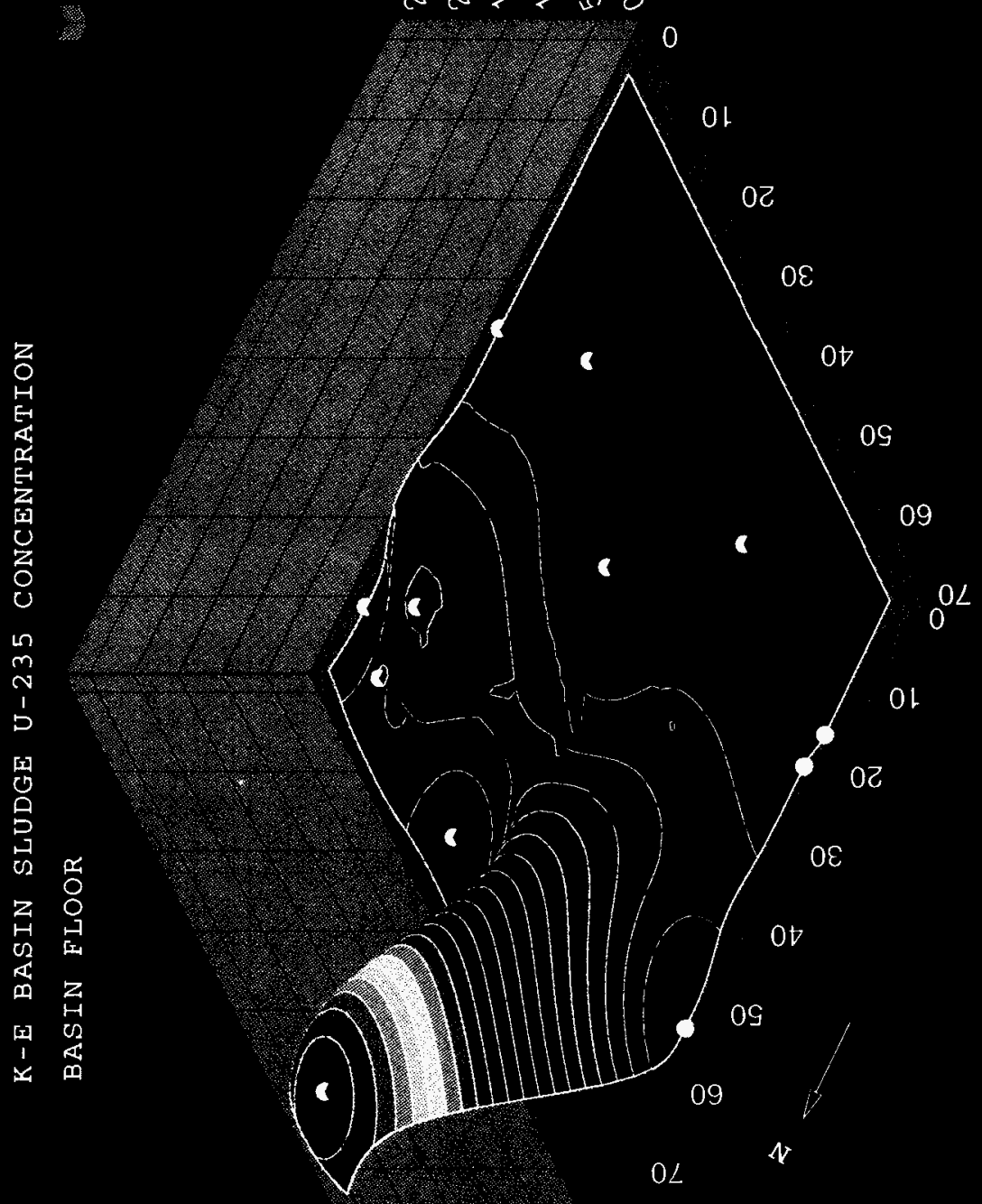

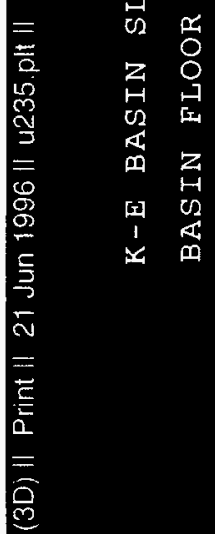

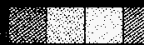




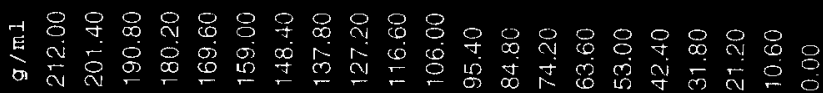
은

桡

$$
\begin{aligned}
& 0.0 .0 .0 .0
\end{aligned}
$$

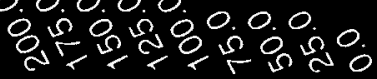

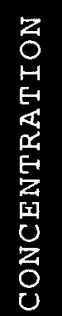

$w$
$m$
$\sim$
1
$p$

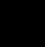

Ir

息

z 8

H

of

4

口吕

国

1
1 


\section{K-E BASIN SLUDGE U-238 CONCENTRATION}

\section{BASIN FLOOR}

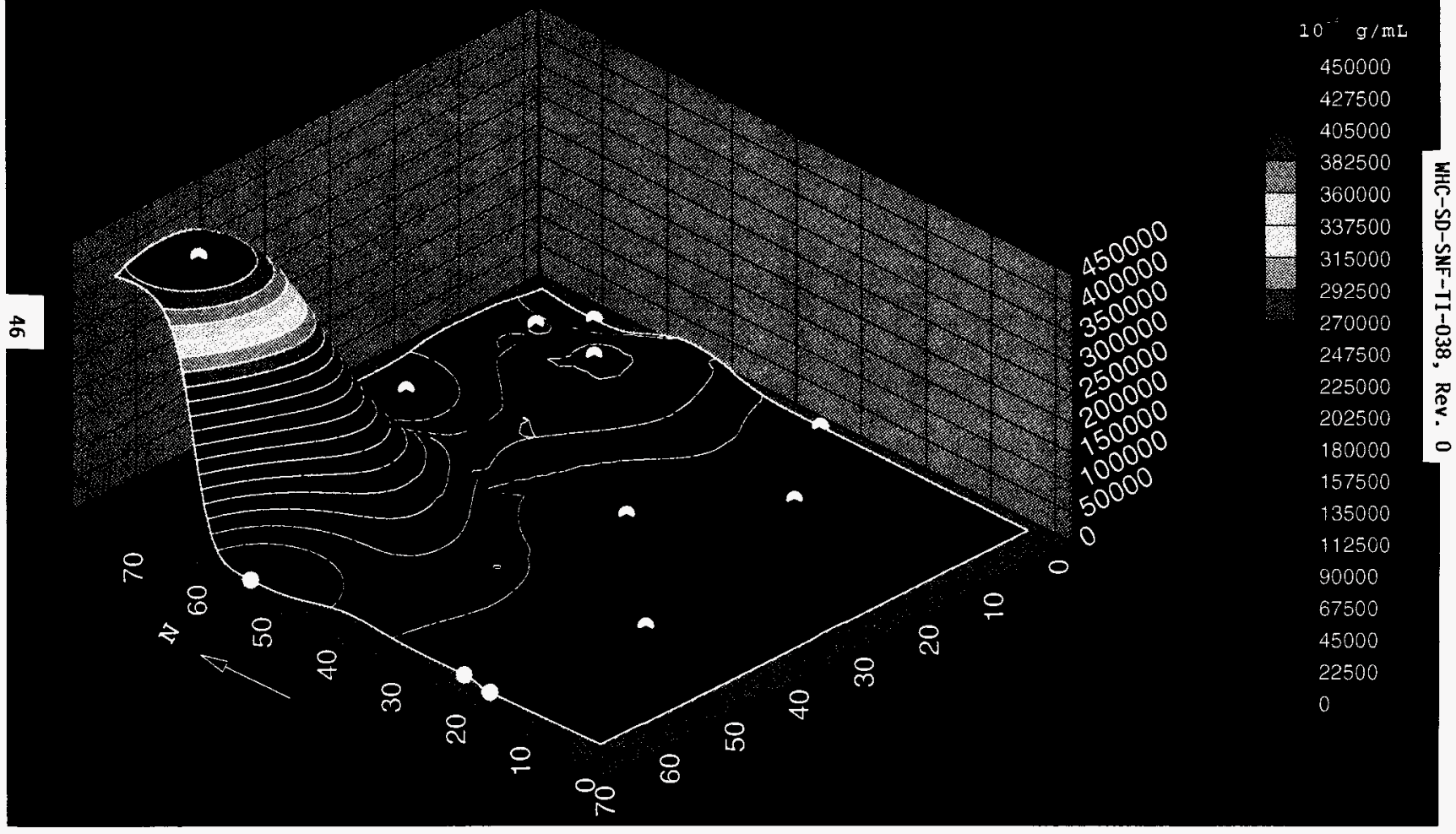




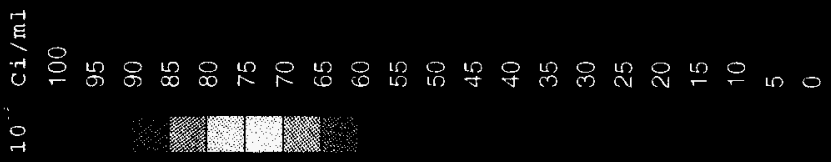

14

$$
898 \% 90
$$

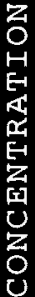

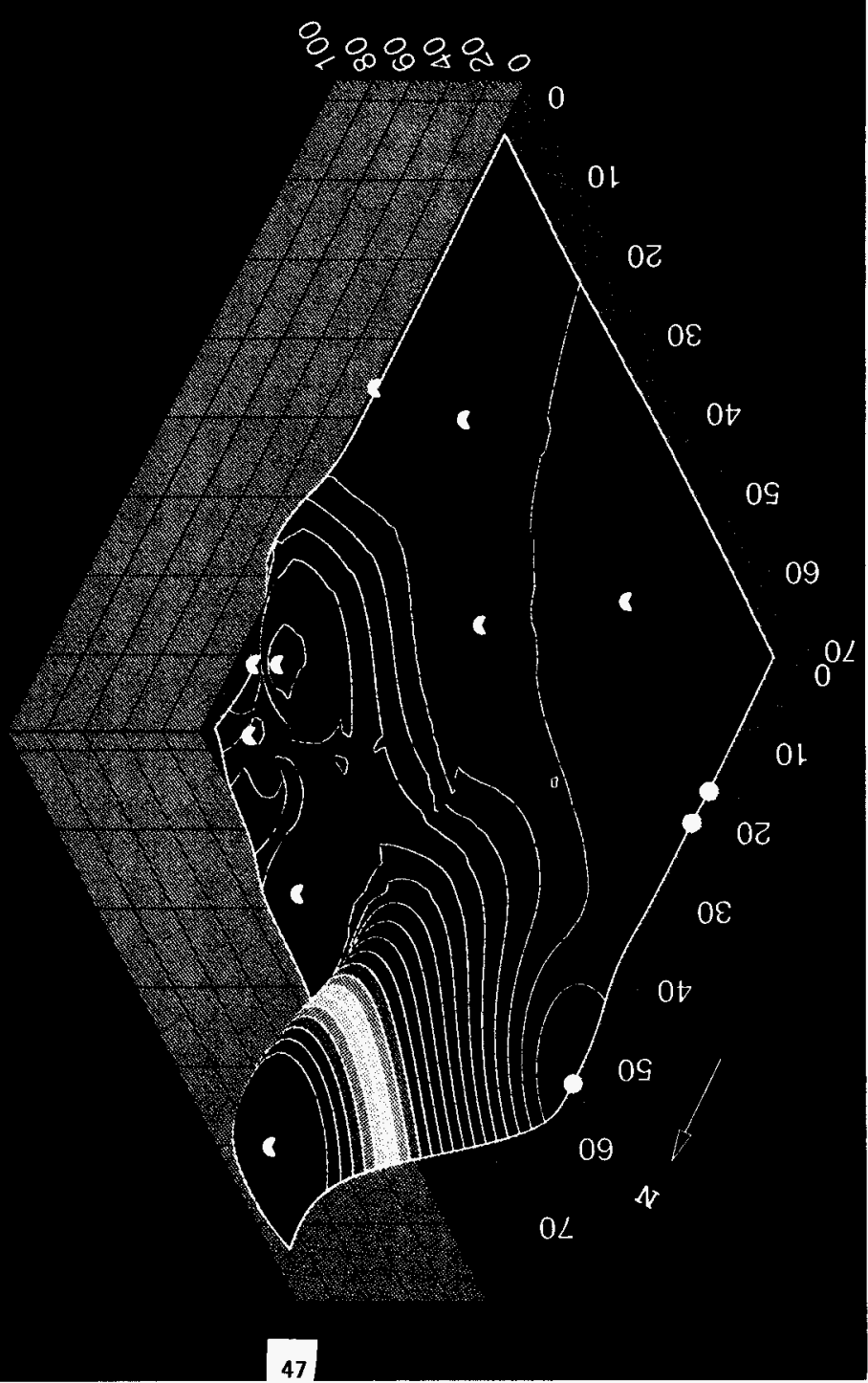

4

田

군

봉

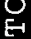

또

음

吕

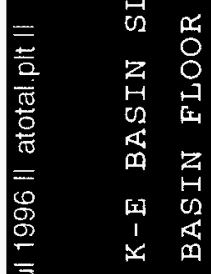




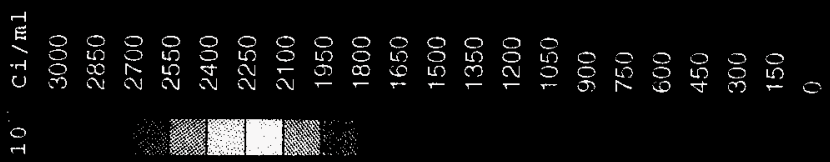

\&

$$
{ }_{8} g_{0} g_{1} g_{8}
$$

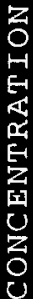

E

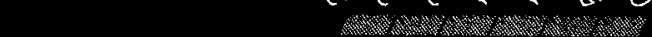
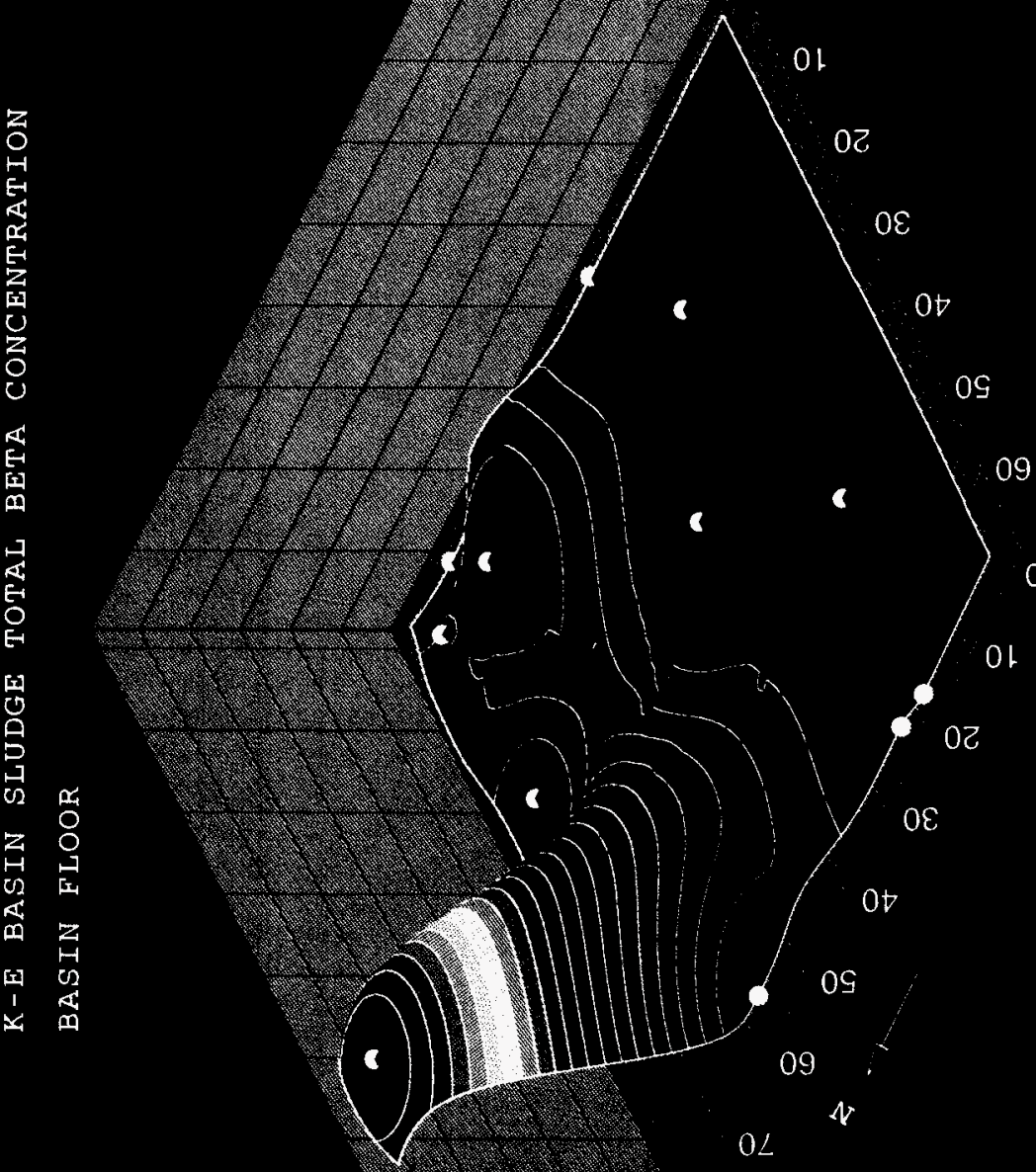

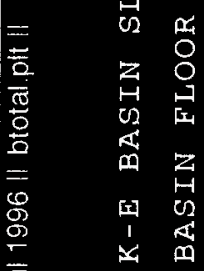

3

กี

$=$

莫

* .

1) 
K-E BASIN SLUDGE Ag CONCENTRATION

\section{BASIN FLOOR}

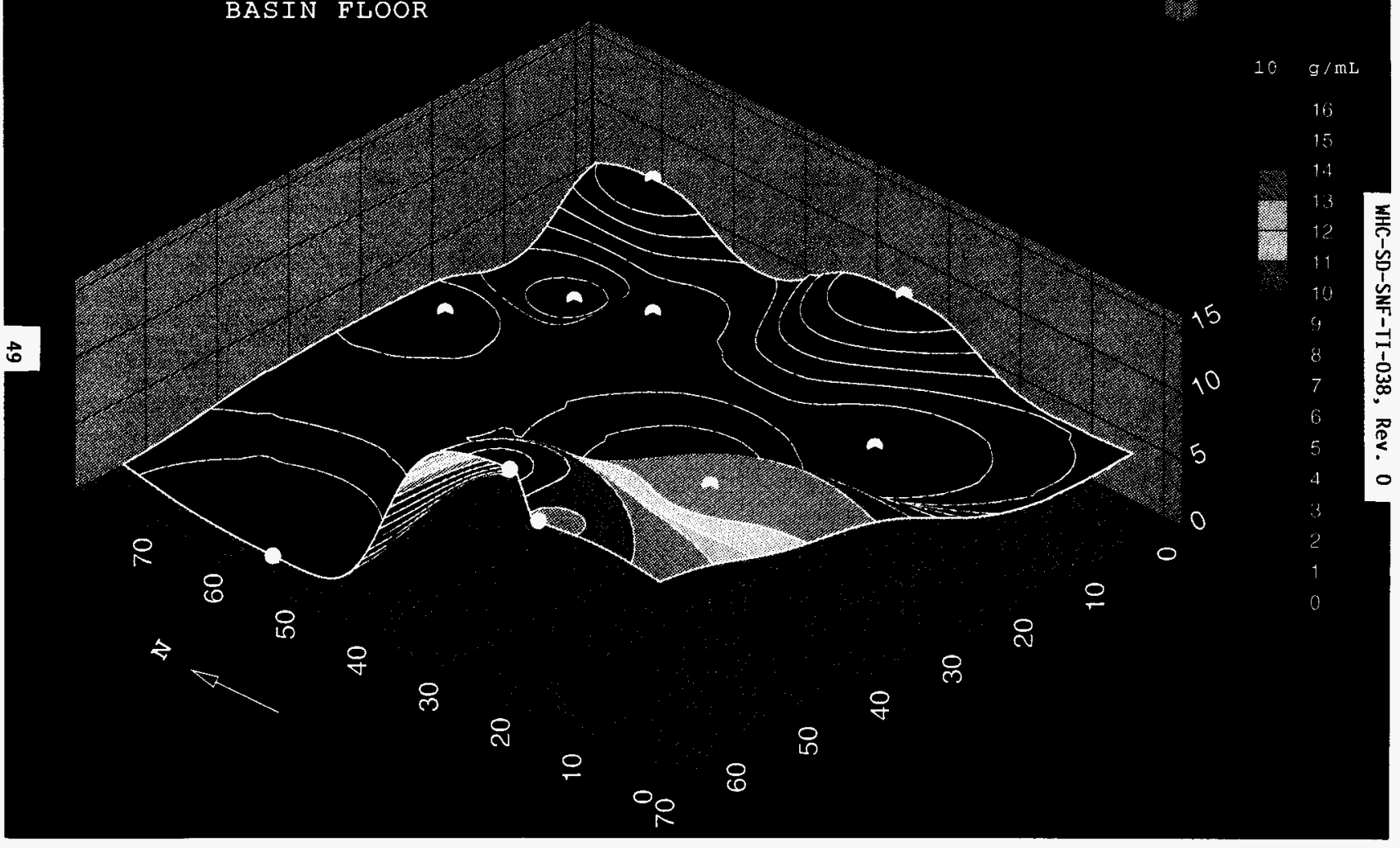


(3D) || Print || 19 Jun 1996 || Algrid.plt || Al Concentration

K-E BASIN SLUDER TI GNCENTRATION

BASIN FLOON

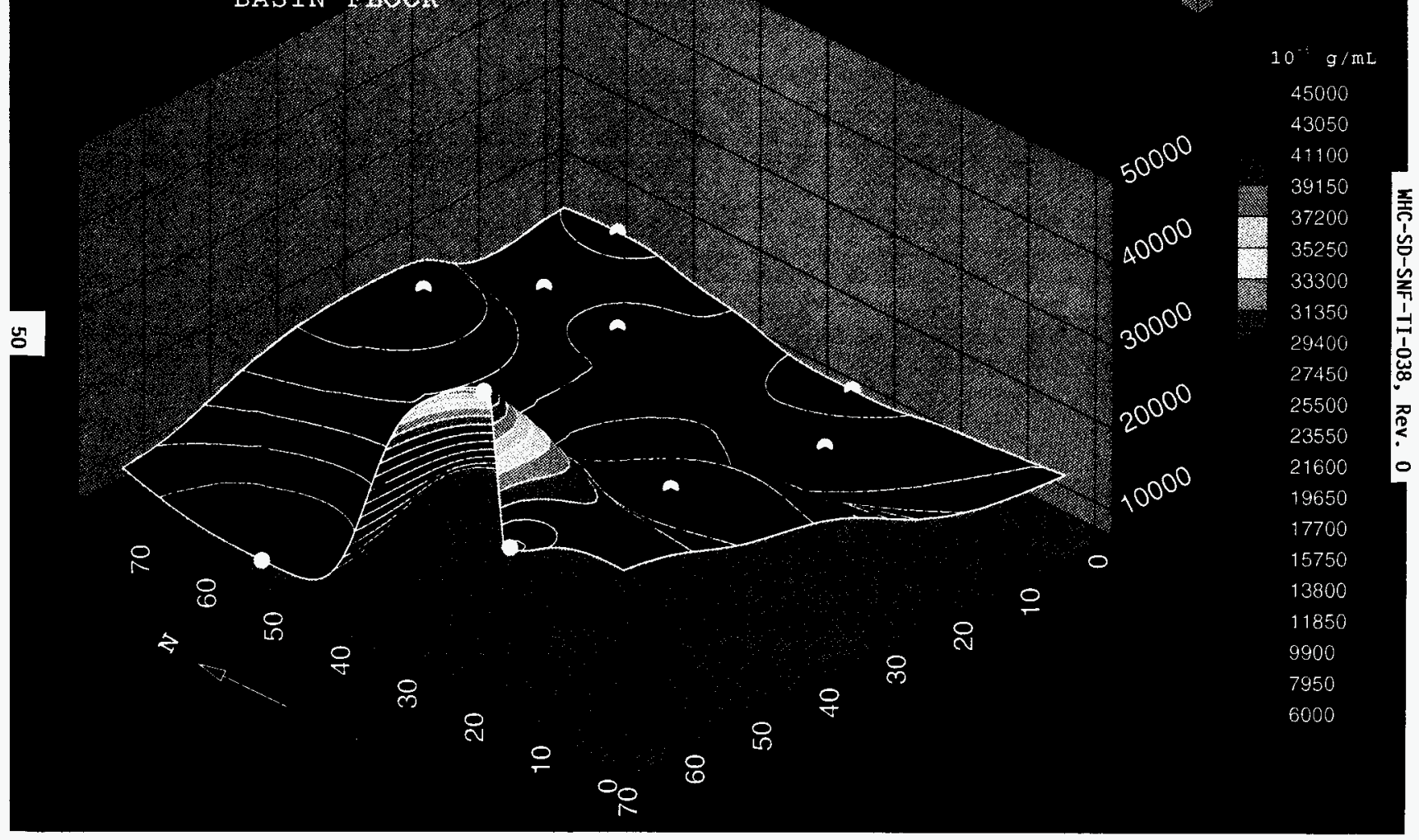




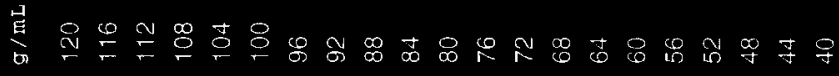

$$
\begin{aligned}
& \stackrel{\circ}{\rightarrow}
\end{aligned}
$$

\%

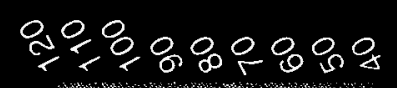

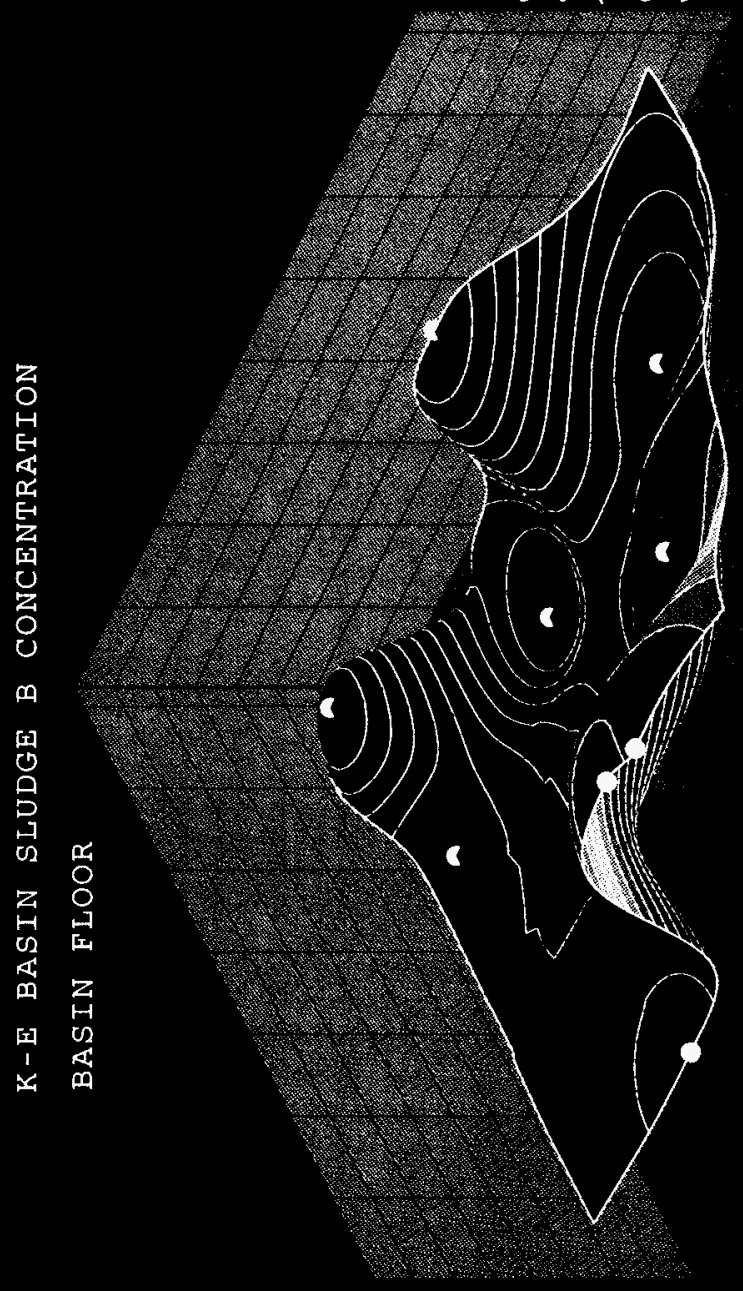

0

01

02

OE

ot 
K-E BASIN SLUDGE Ba CONCENTRATION

BASIN FLOOR

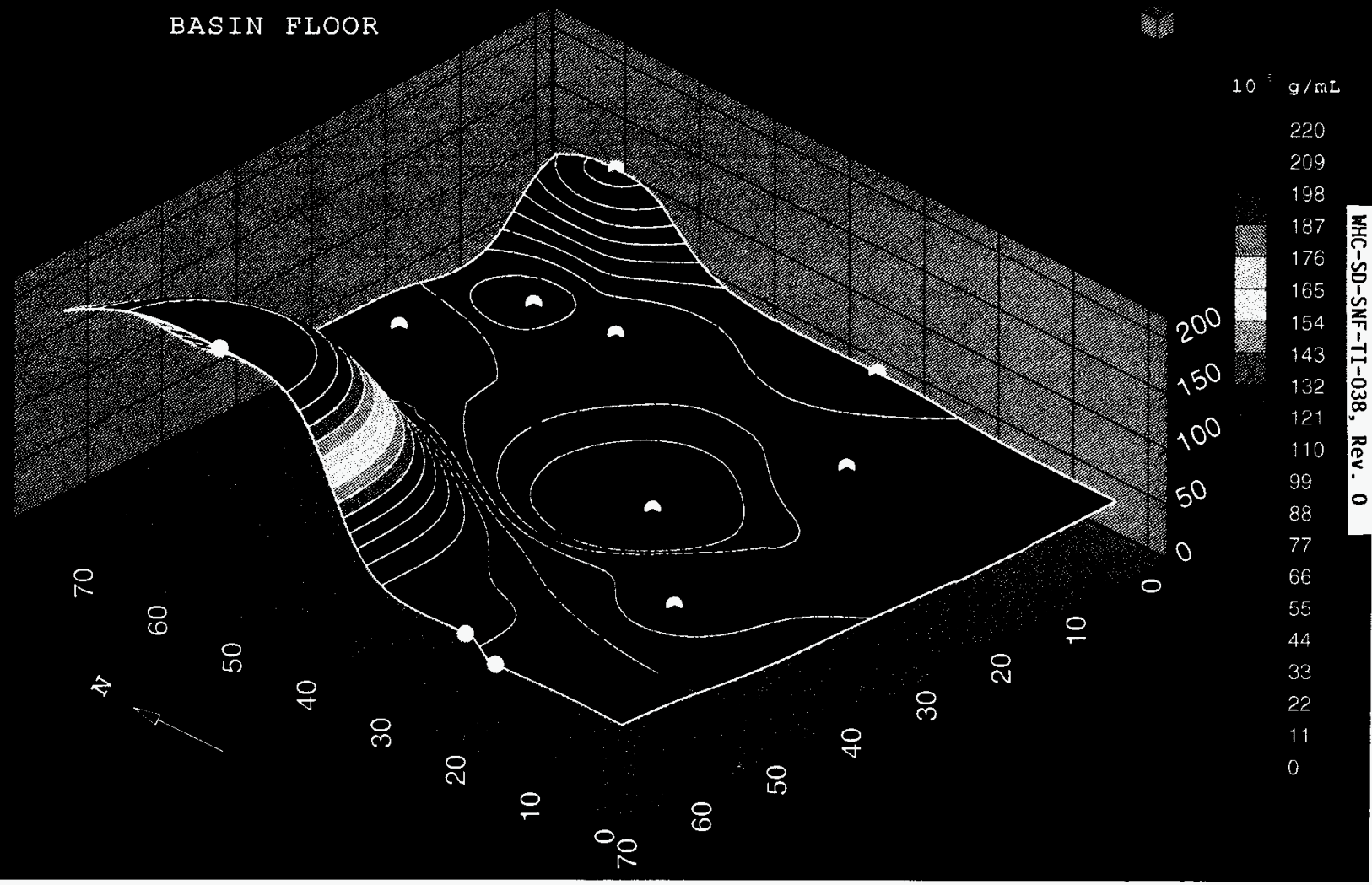


WHC-SD-SNF-TI-038, Rev. 0

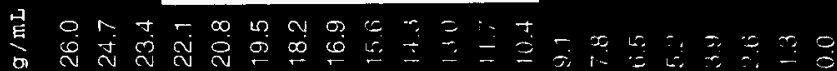

욱

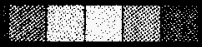

$$
\text { singo }
$$

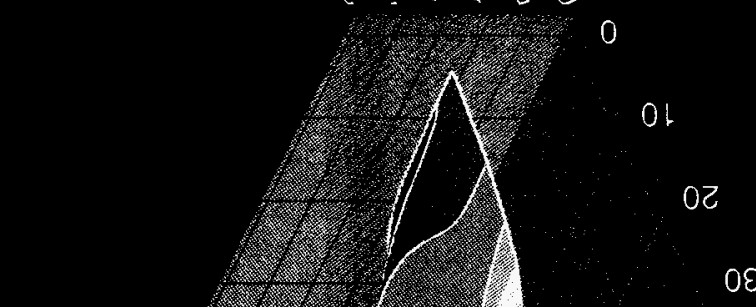

3
0
0
0
0
0
0
0
0
0
0
0
0

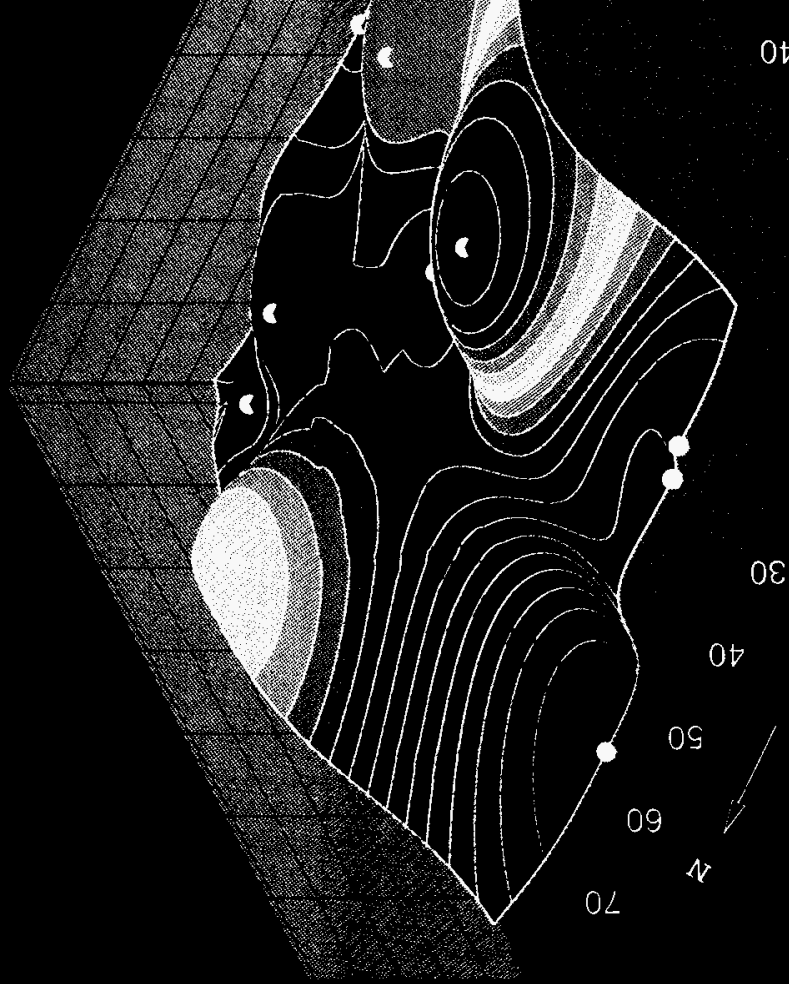

09

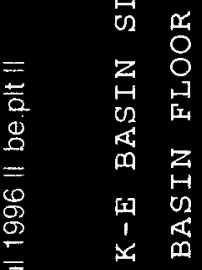




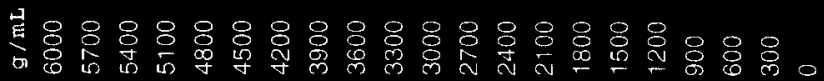
0

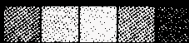

稵

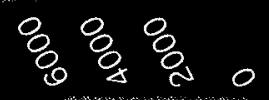

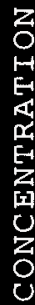

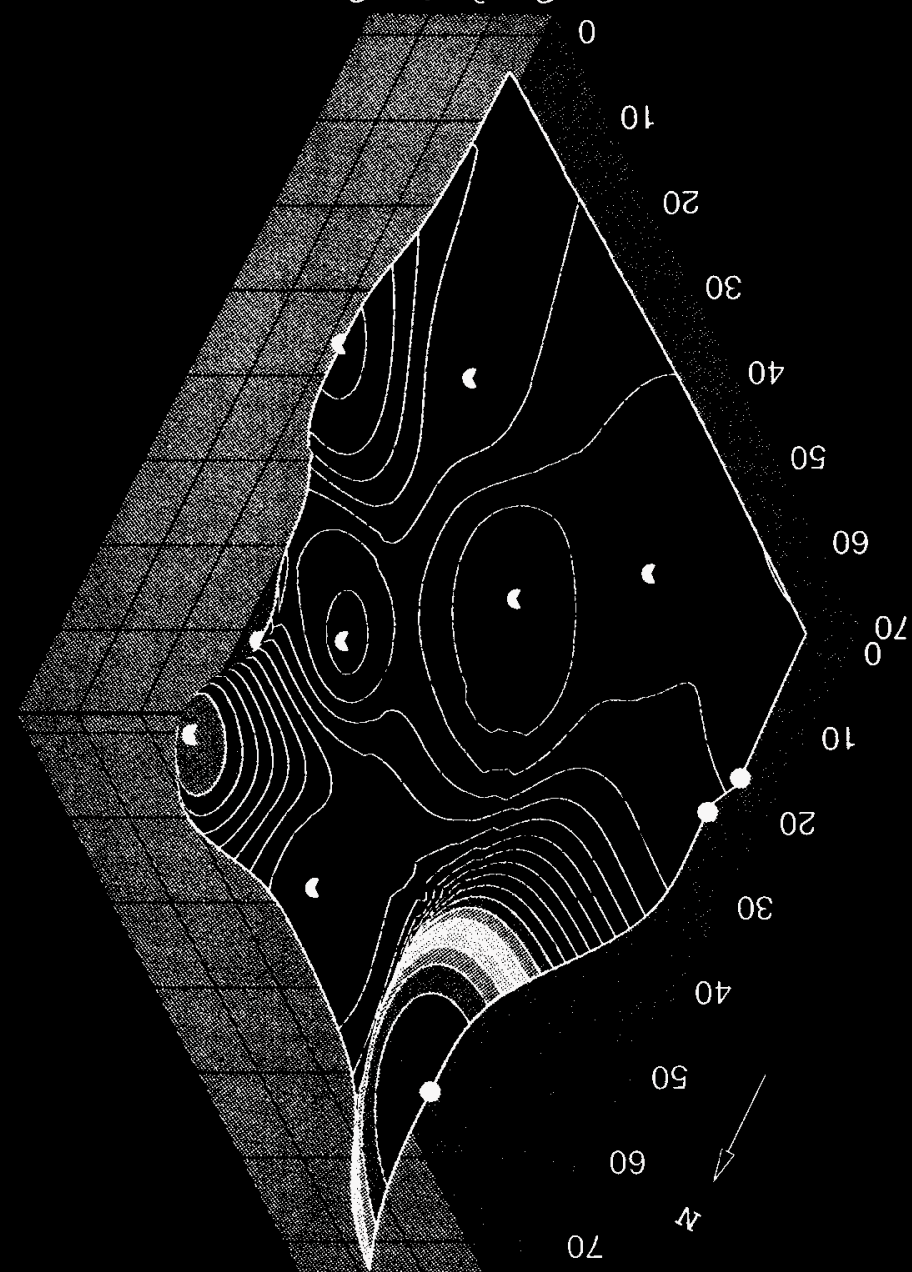

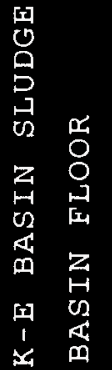




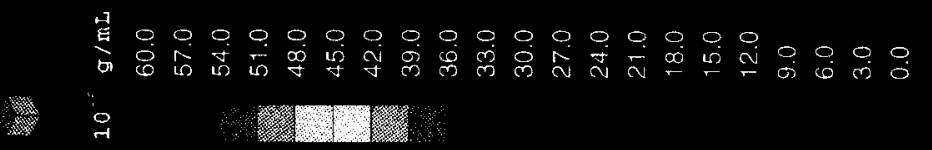

\section{$\because \& \& ; \% ? 0$}

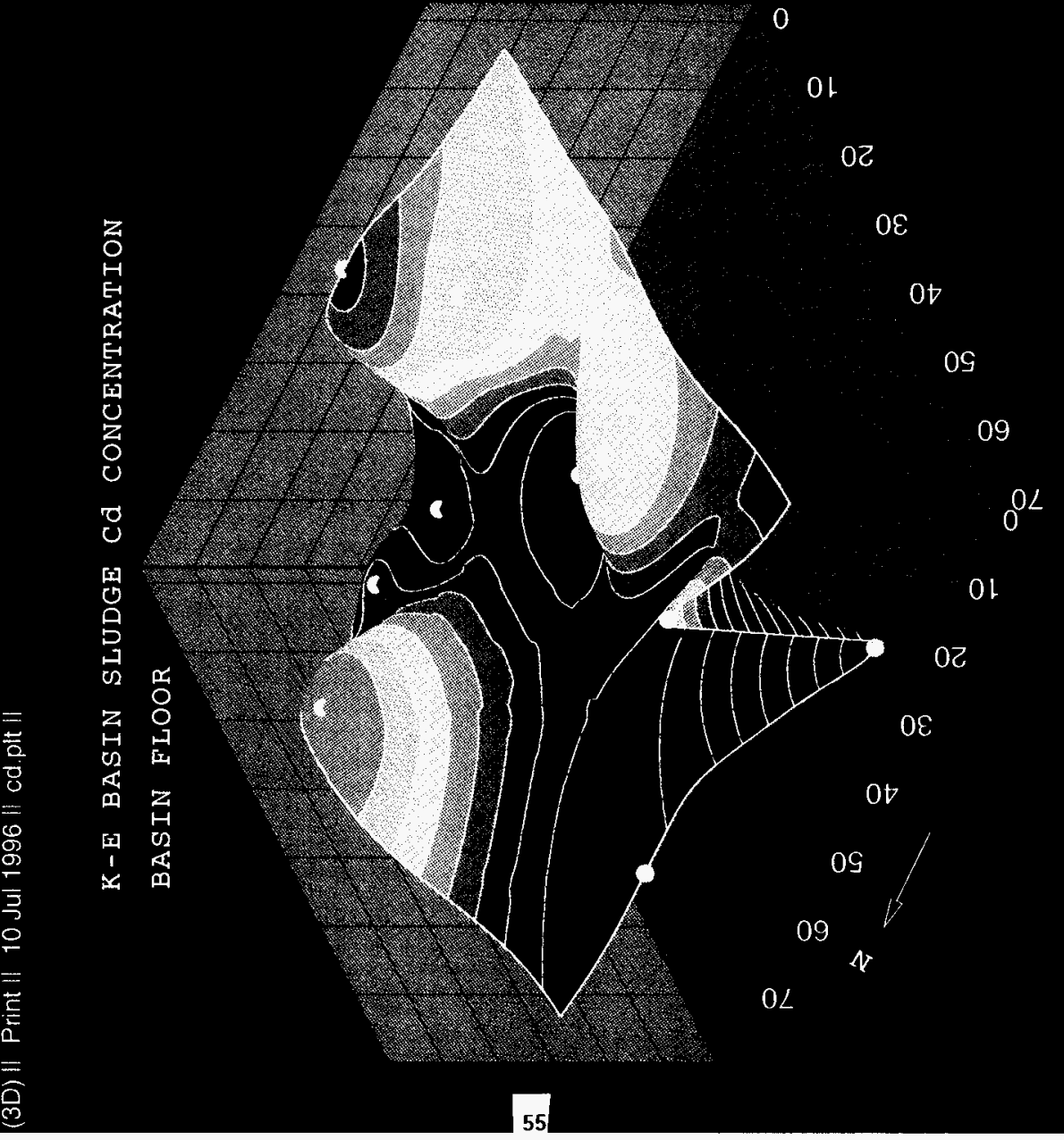




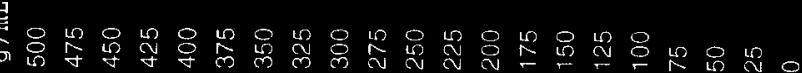

ल

的宫 8

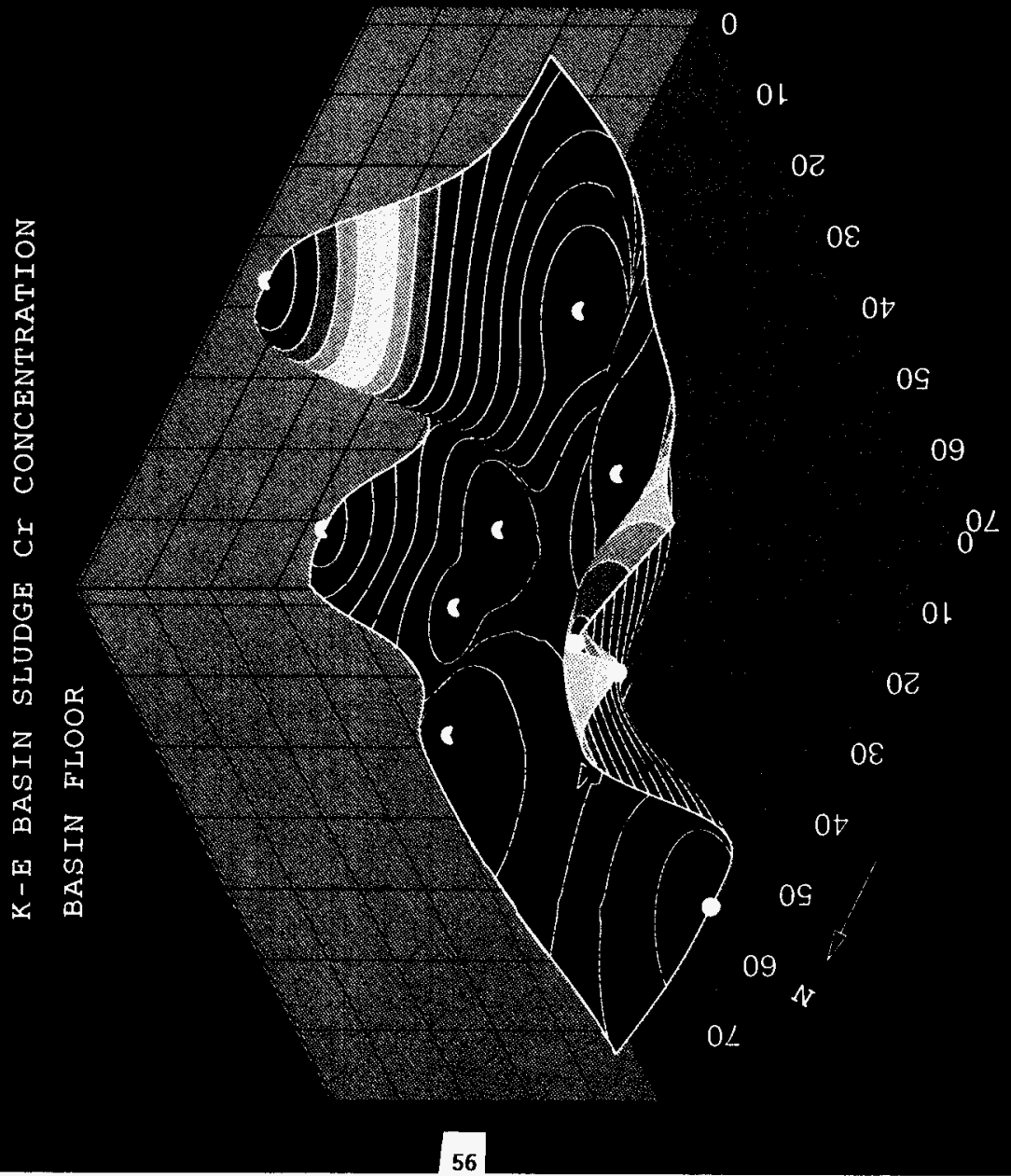


K-E BASIN SLUDGE CU CONCENTRATION BASIN FLOOR

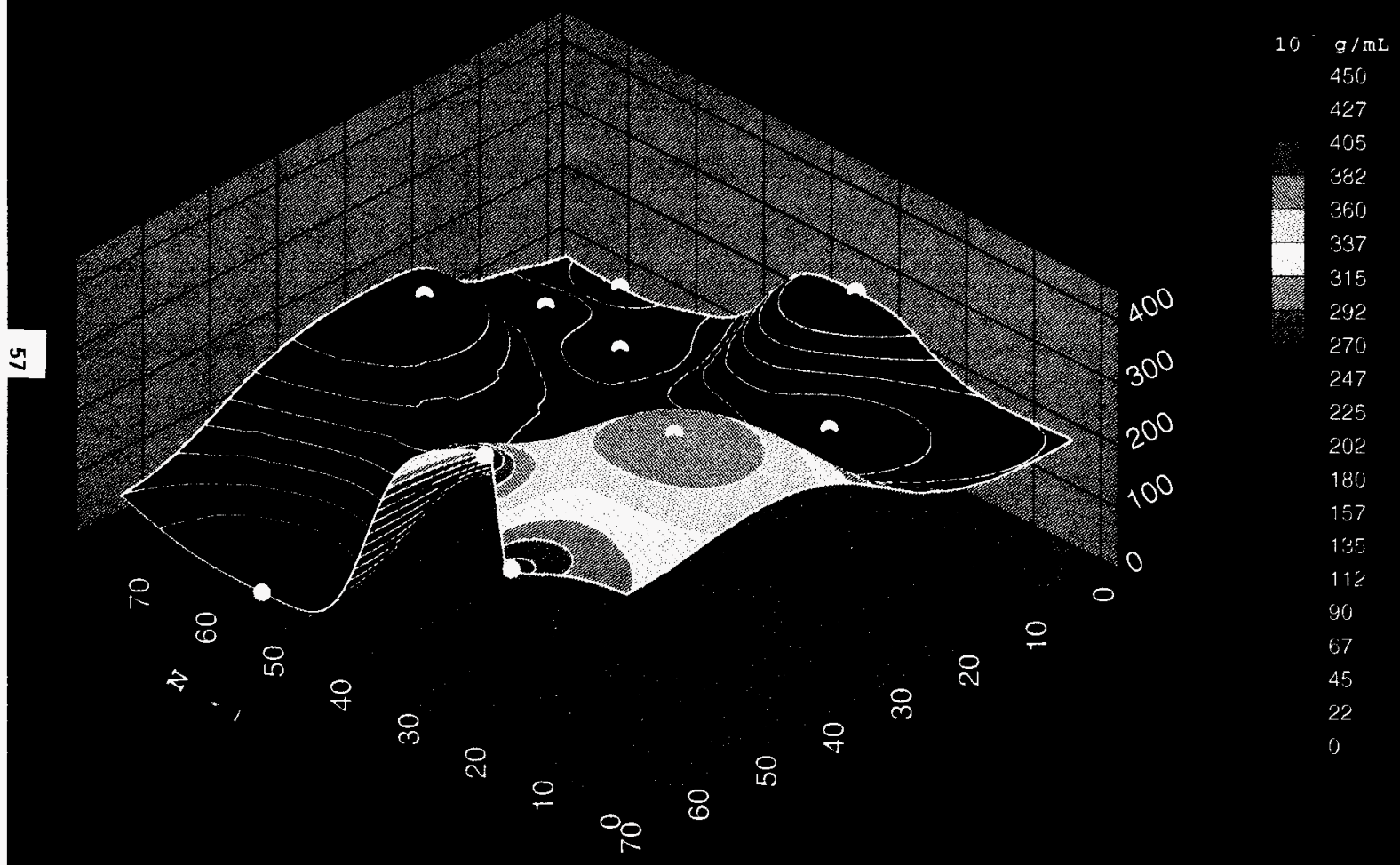




\section{K-E BASIN SLUEER FO CONCENTRATION}

\section{BASIN FIOOX}

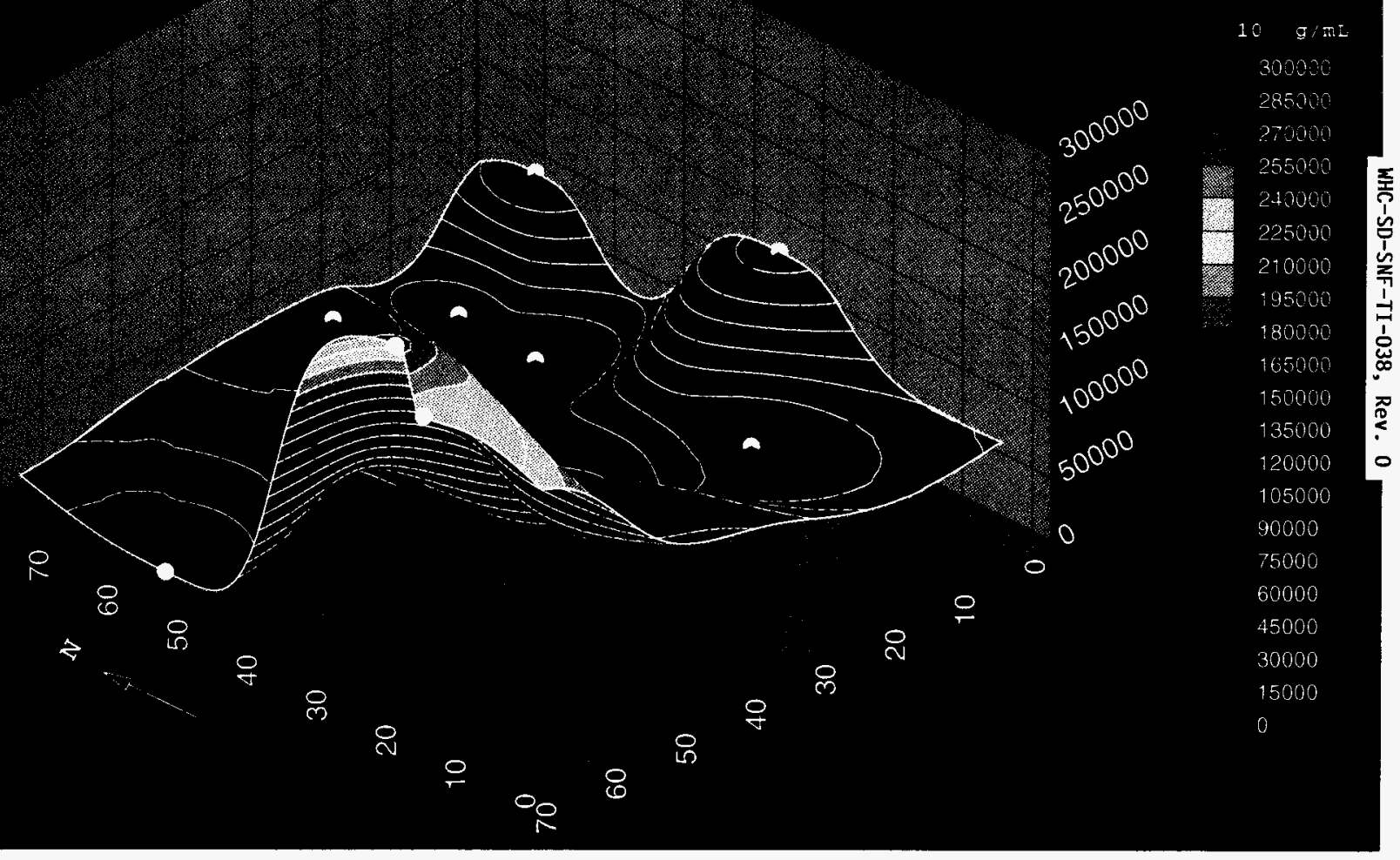


WHC-SD-SNF-TI-038, Rev. 0

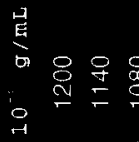

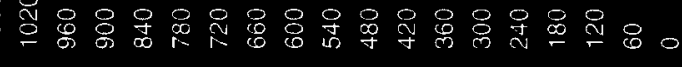
1)

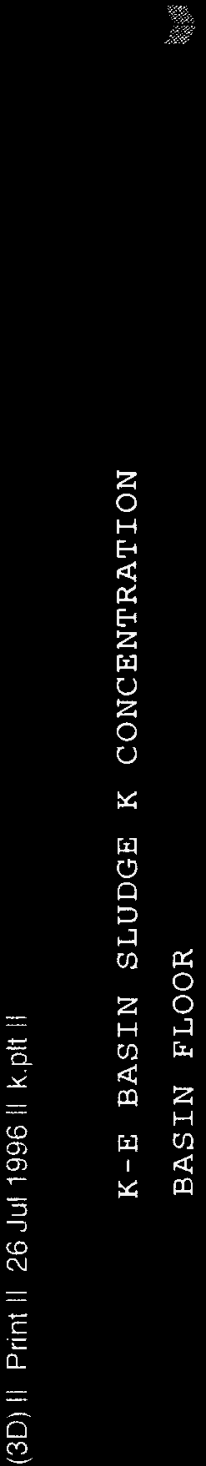

量 8

0

0.

$0 Z$

$0 \varepsilon$

ot

$0 S$ 
WHC-SD-SNF-TI-038, Rev. 0

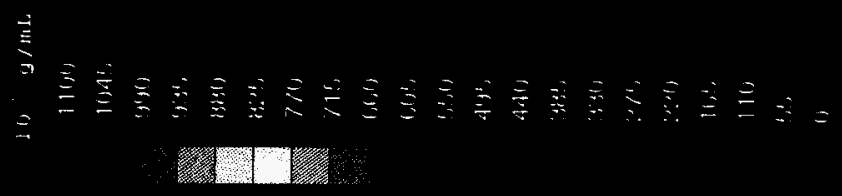

.

$$
8,8388
$$

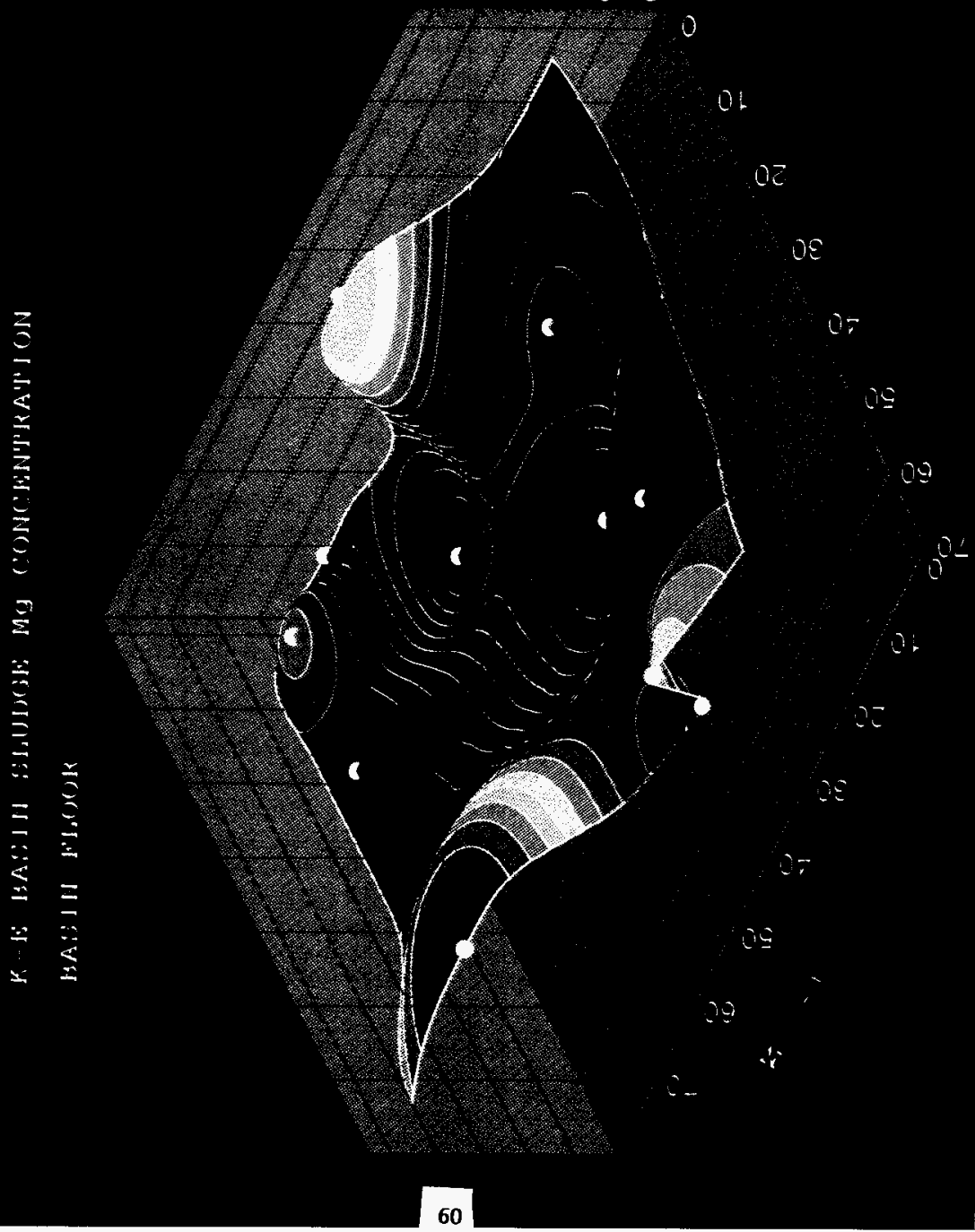


K-E BASIN SLUDGE Mn CONCENTRATION BASIN FLOOR

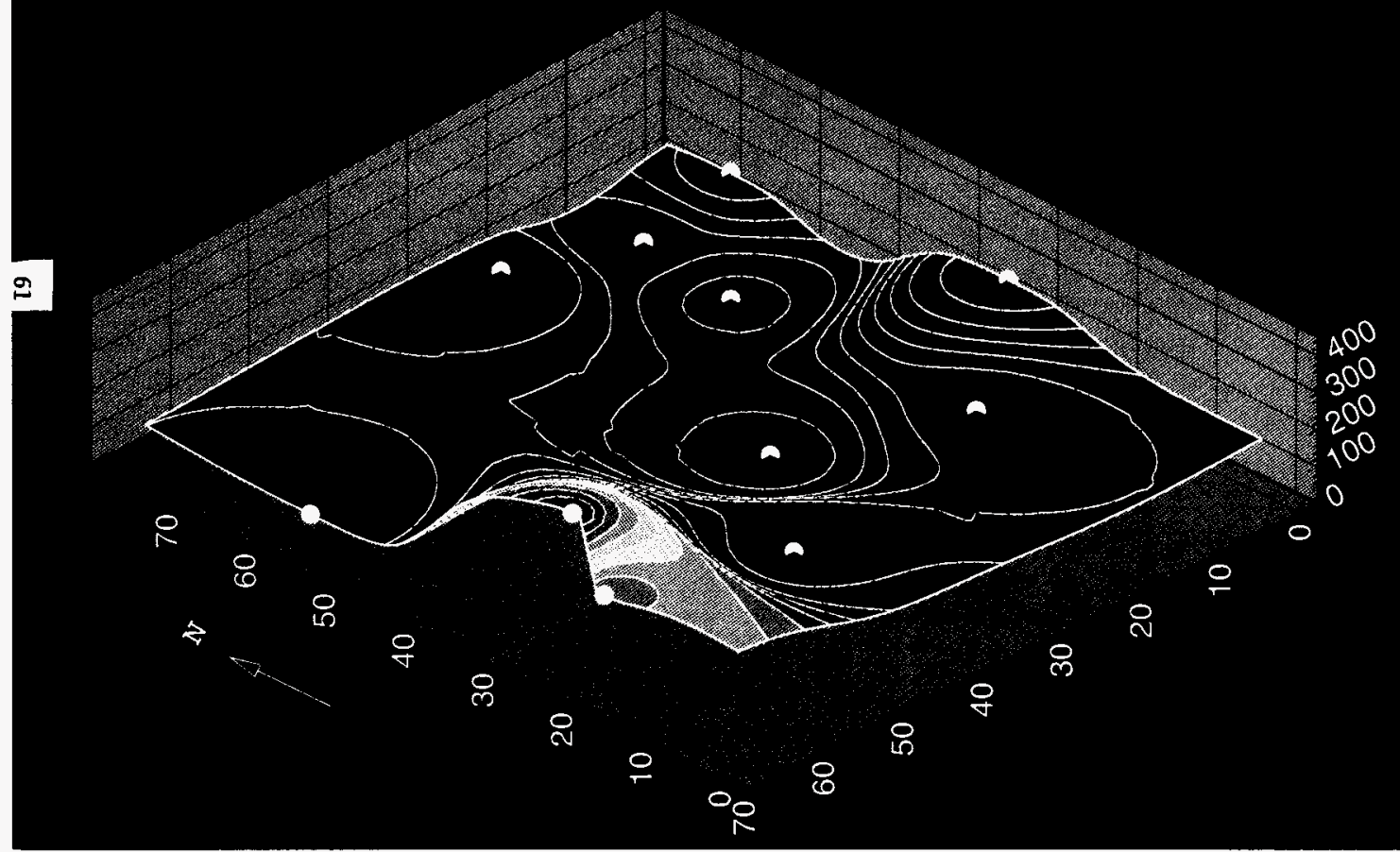


(3D) || Print || 20 Jun 1996 || na.plt ||

K-E BASIN SLUdGe Na CONCENTRATiON

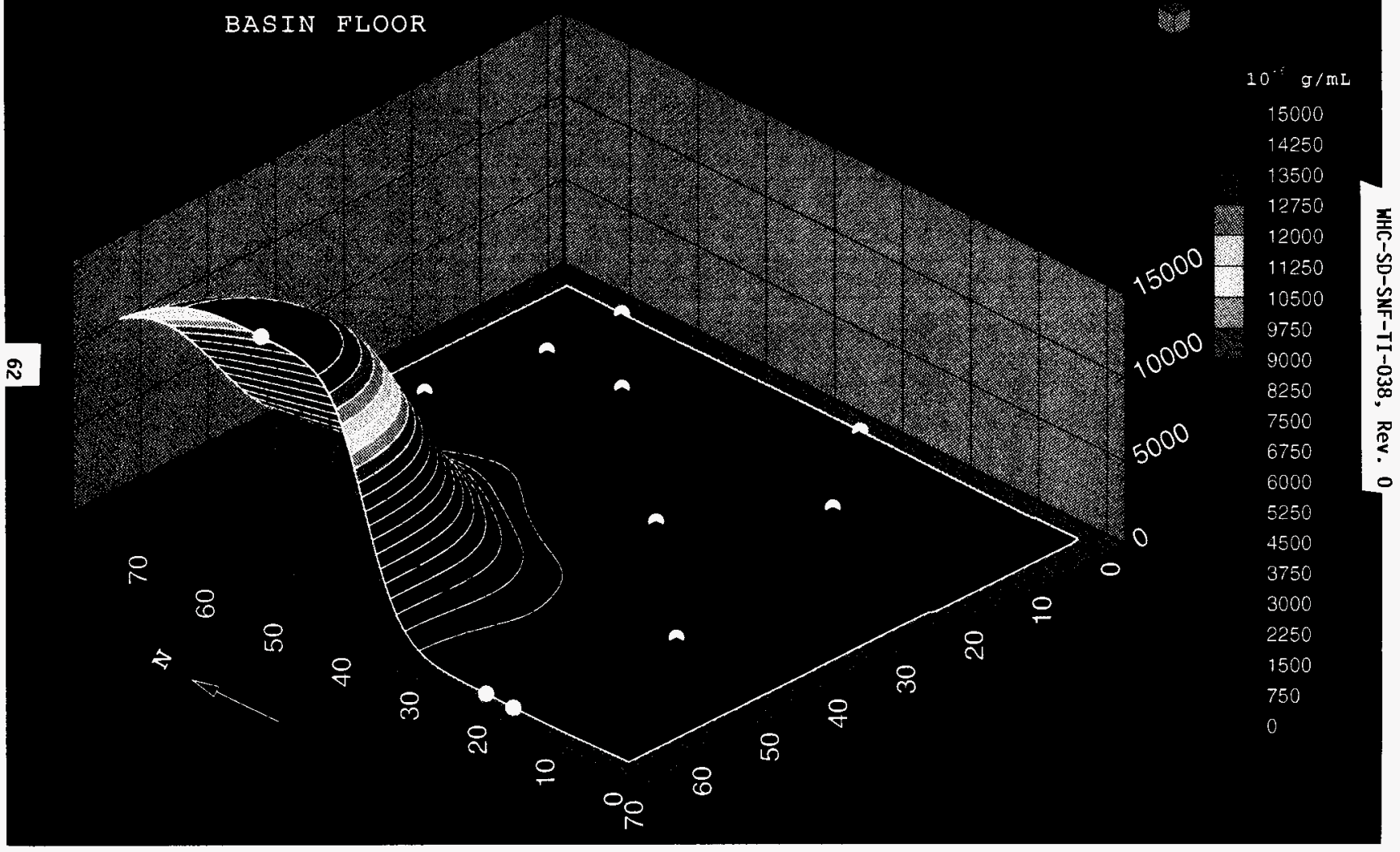




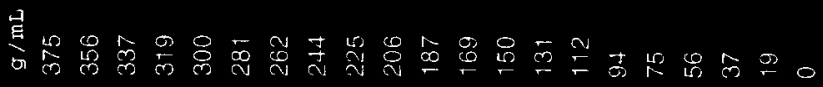

잢

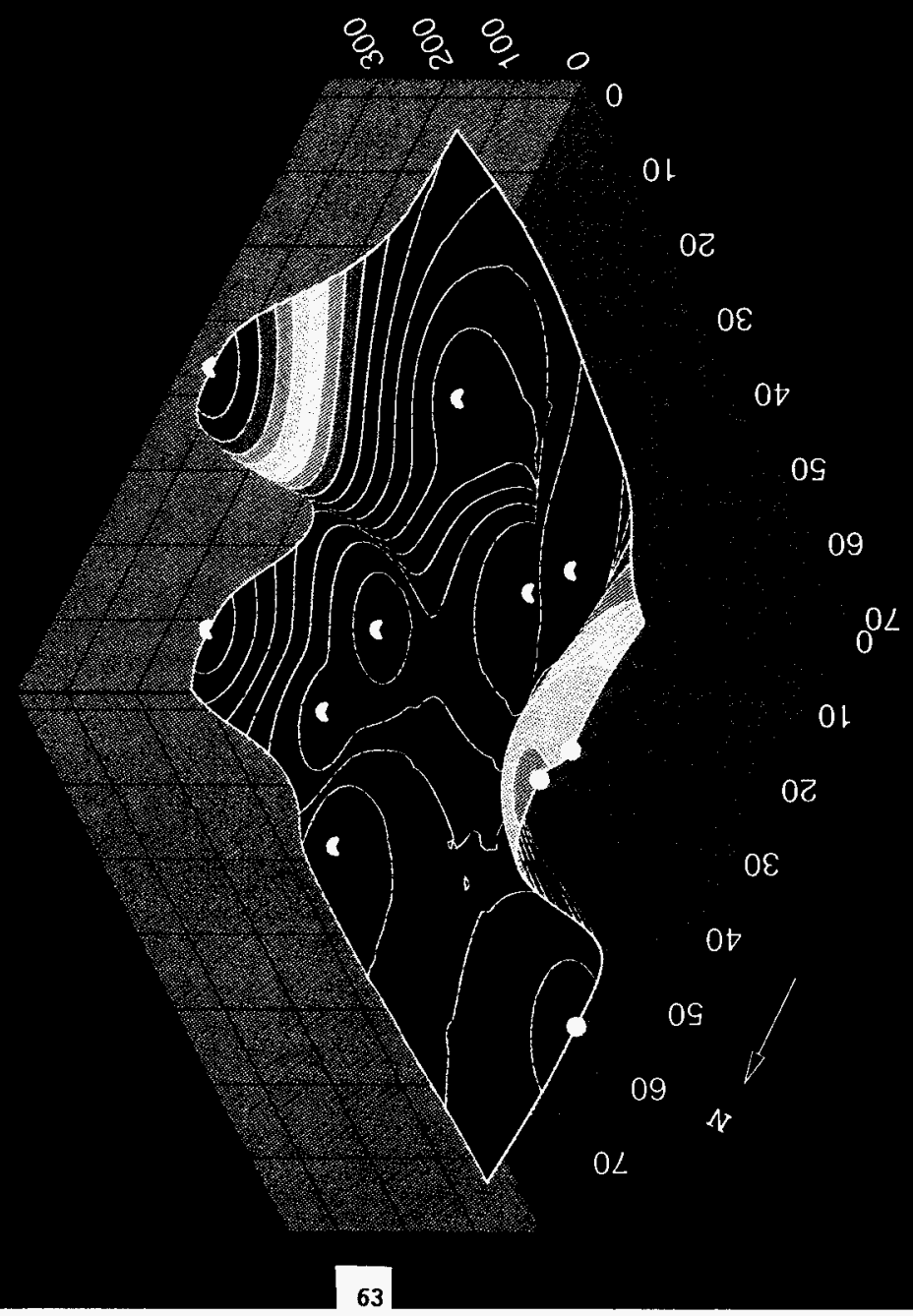


WHC-SD-SNF-TI-038, Rev. 0

$\sum_{0}^{\text {是 }}$

ด

\section{\%}

3x

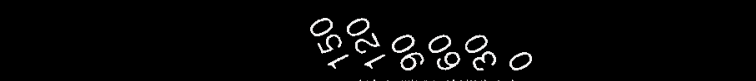

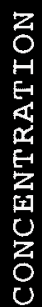
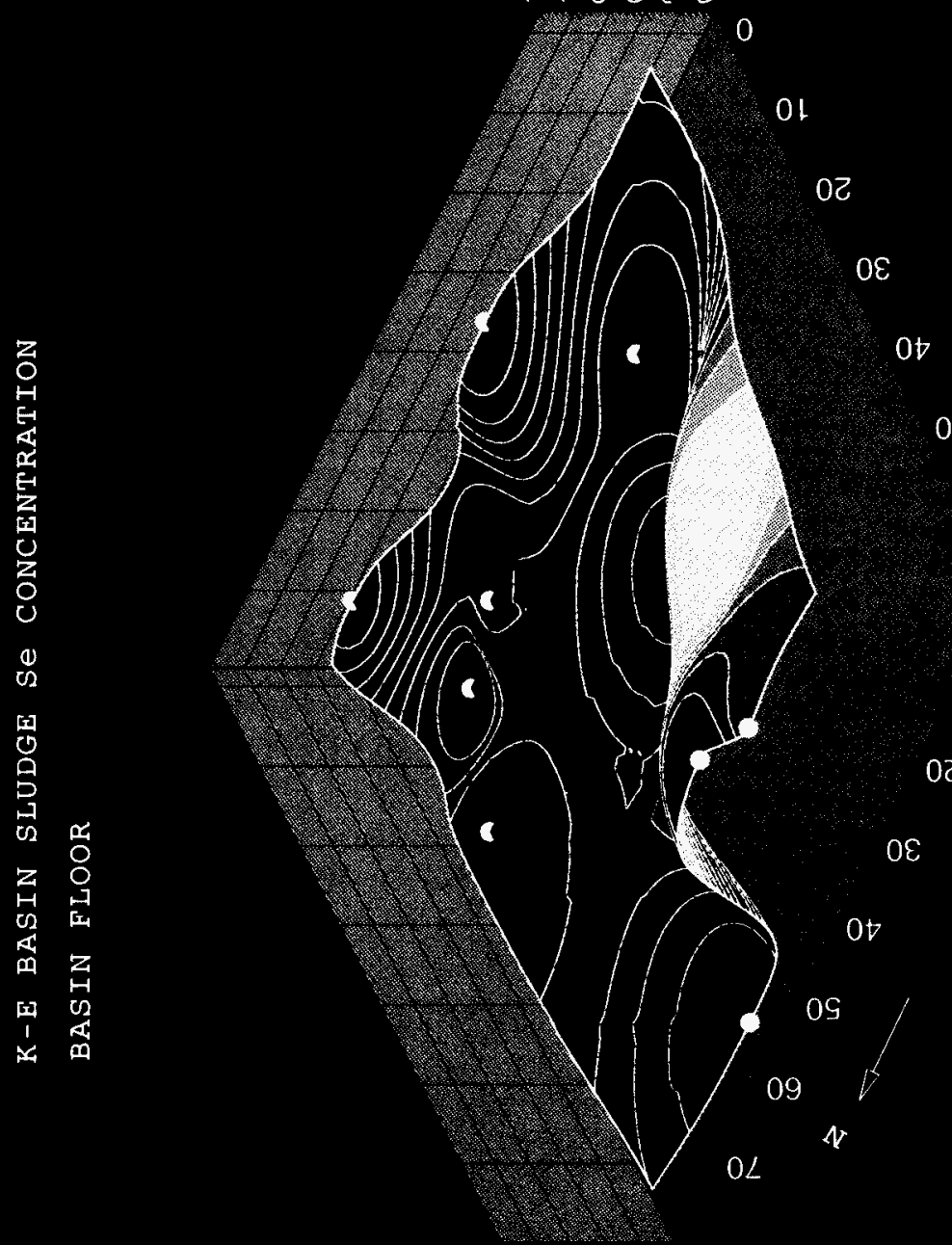

$0 \mathrm{~S}$ 


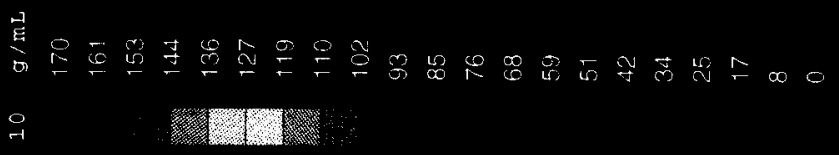

x

$$
\text { \& } 8 \text { is o }
$$

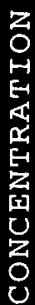

貝

m

0

O

号

的电

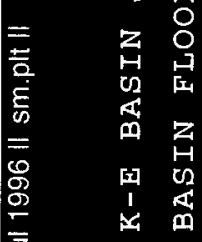

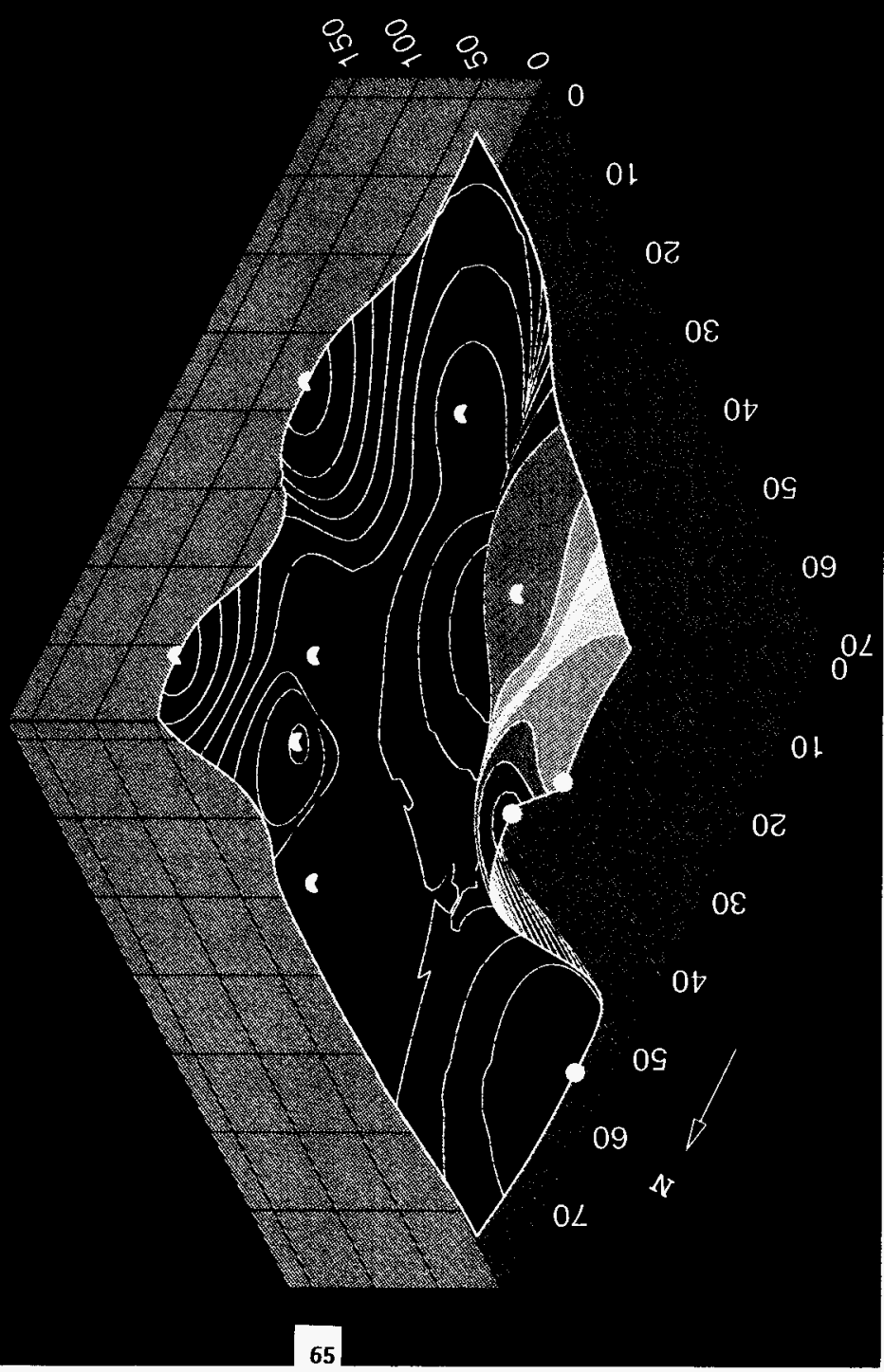


崖

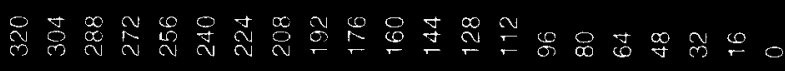

(1)

W

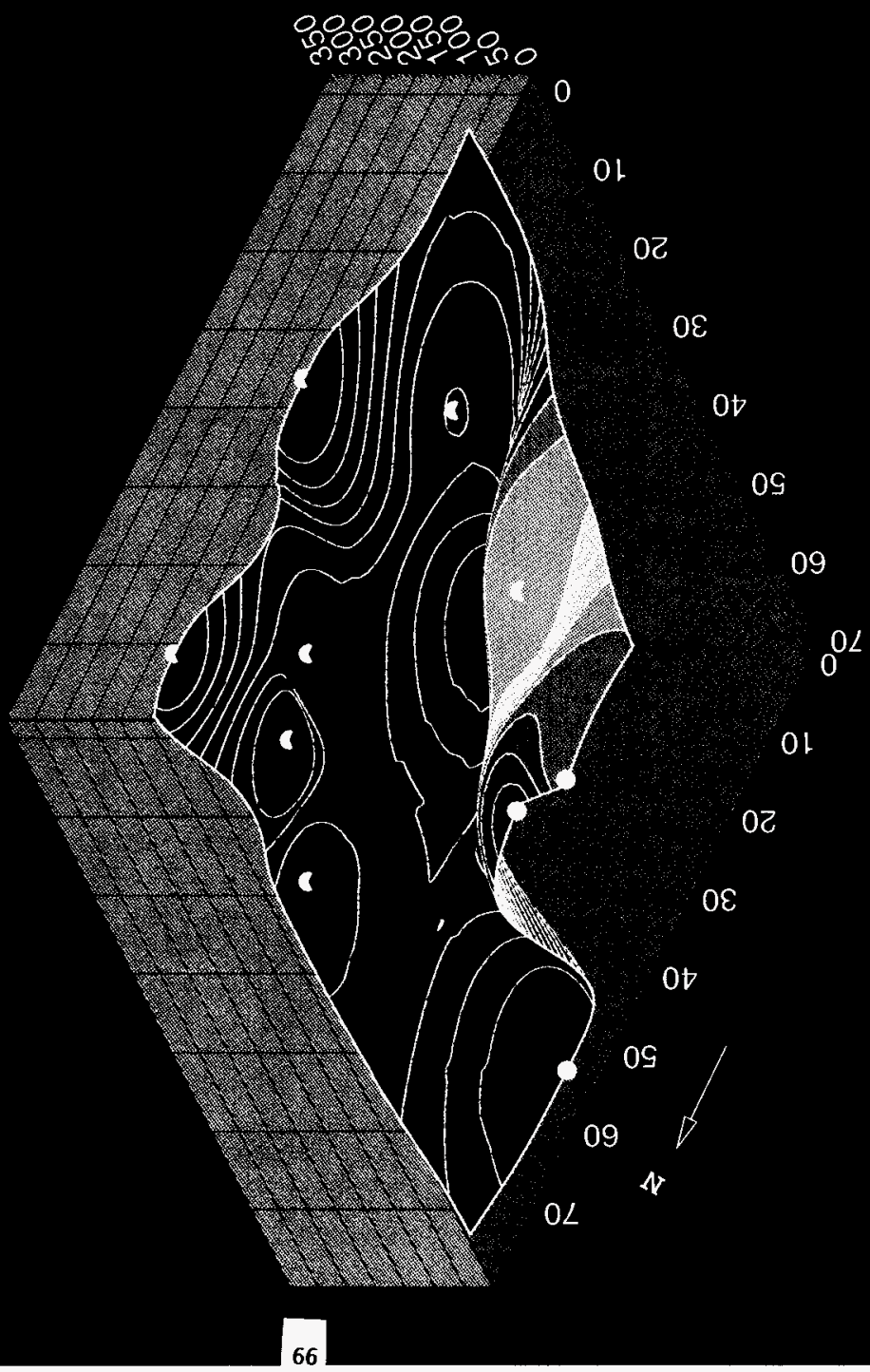




\section{K-E BASIN SLUDGE Zn CONCENTRATION}

\section{BASIN FLOOR}

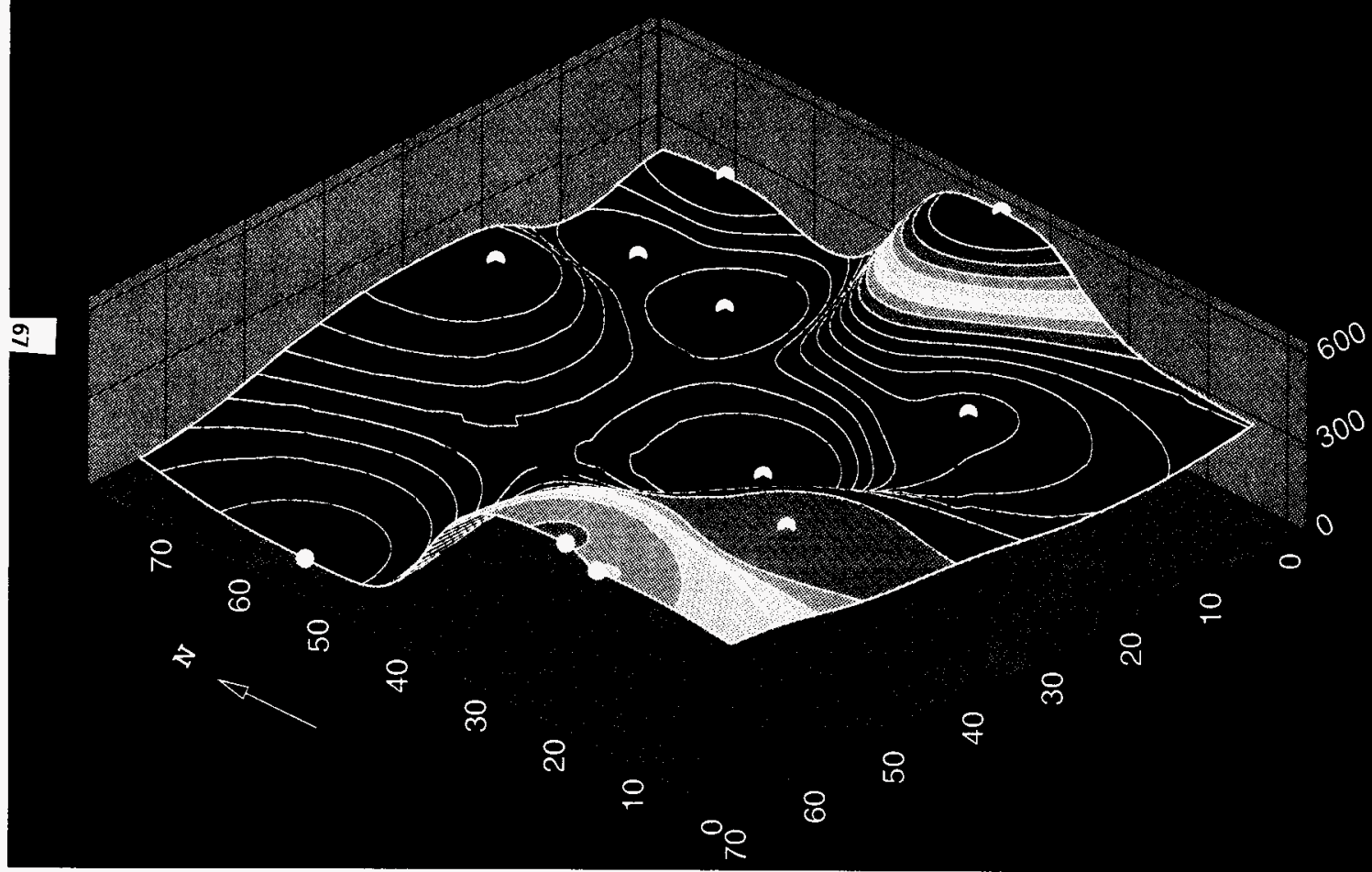

$10 \mathrm{~g} / \mathrm{mL}$ 650 617 

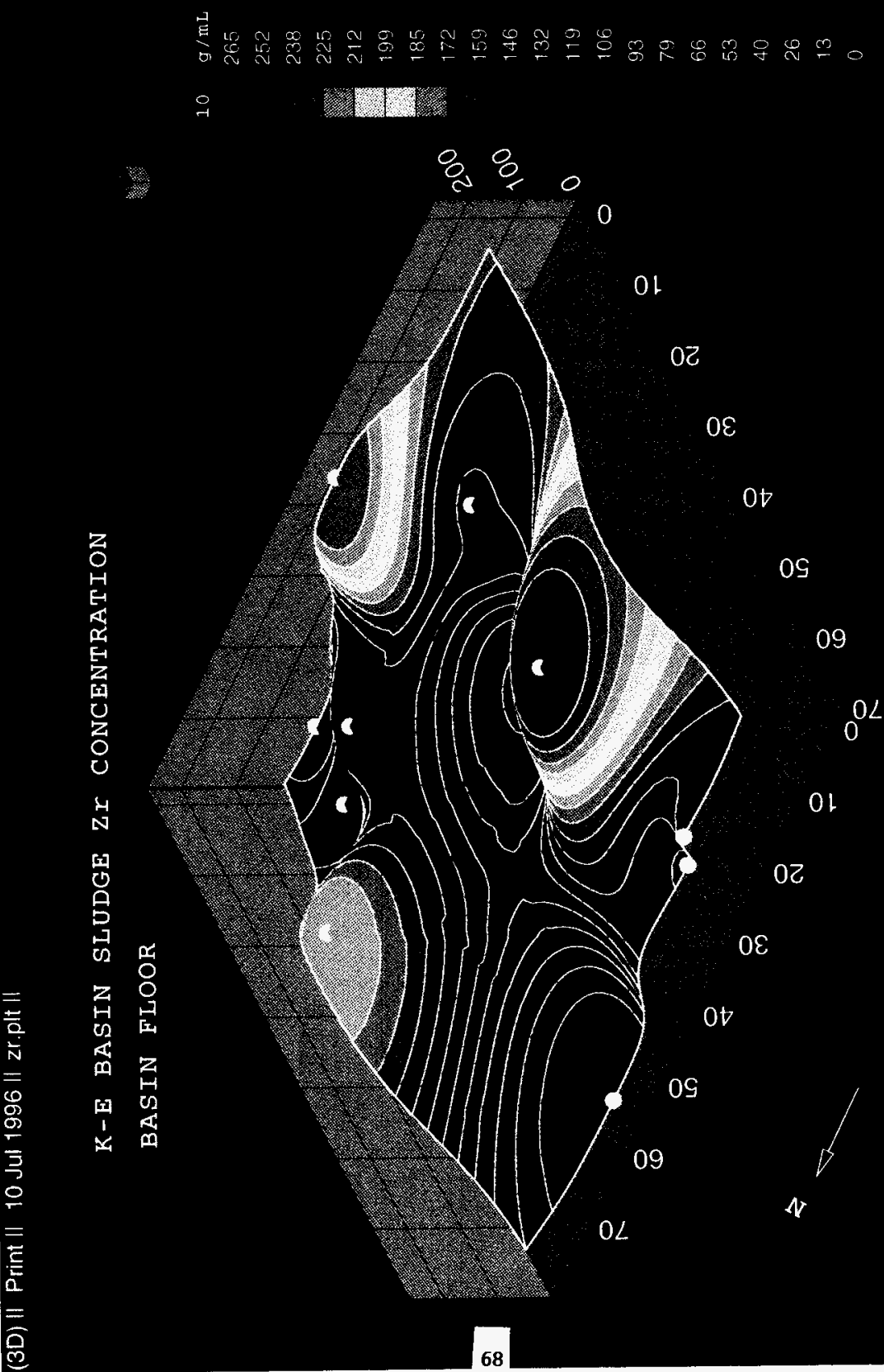
(3D) || Print || 26 Jul 1996 || tic.plt ||

K-E BASIN SLUDGE TOTAL INORGANIC CARBON CONCENTRATION BASIN FLOOR

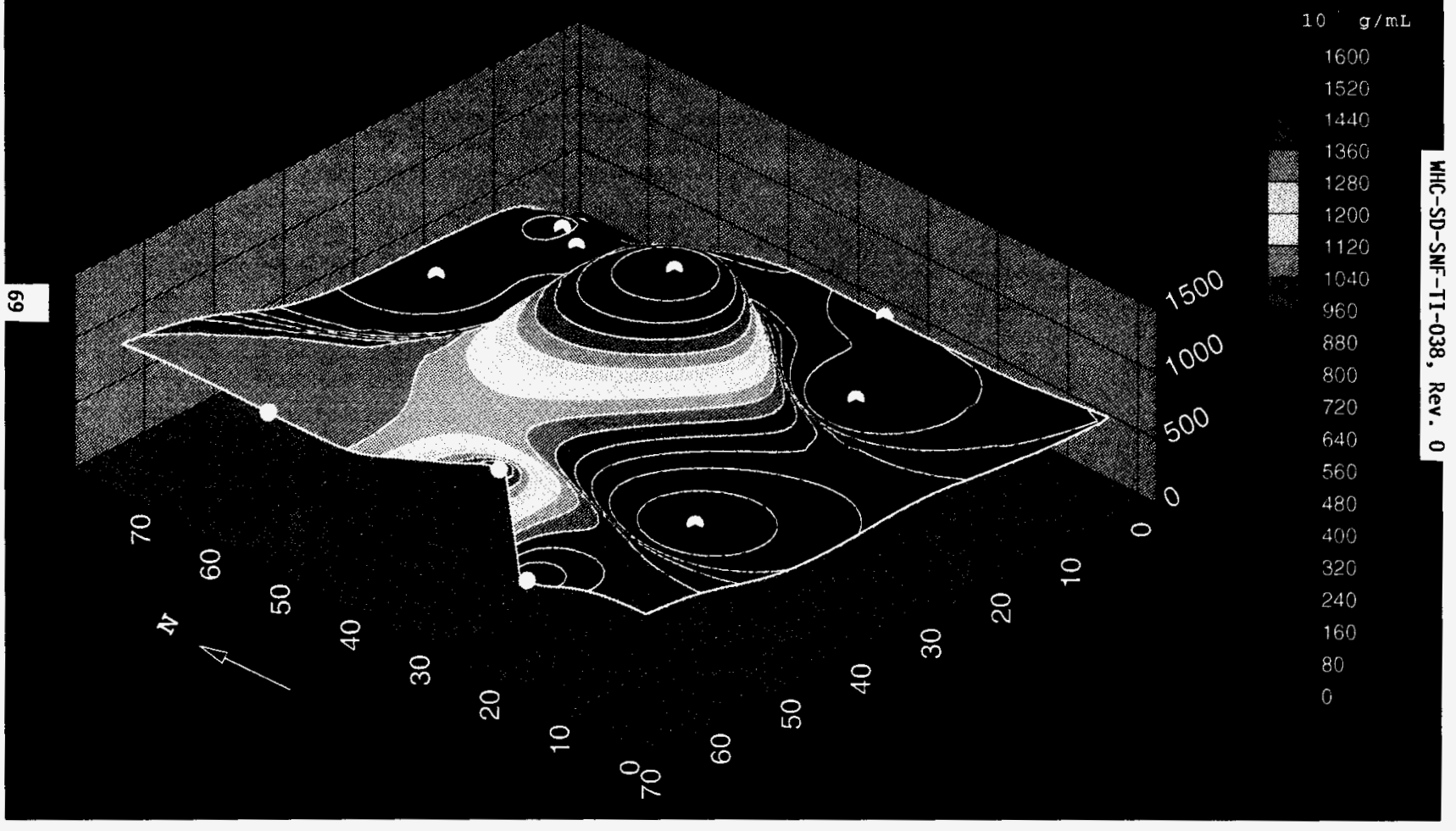


K-E BASIN SLUDGE TOTAL ORGANIC CARBON CONCENTRATION

\section{BASIN FLOOR}

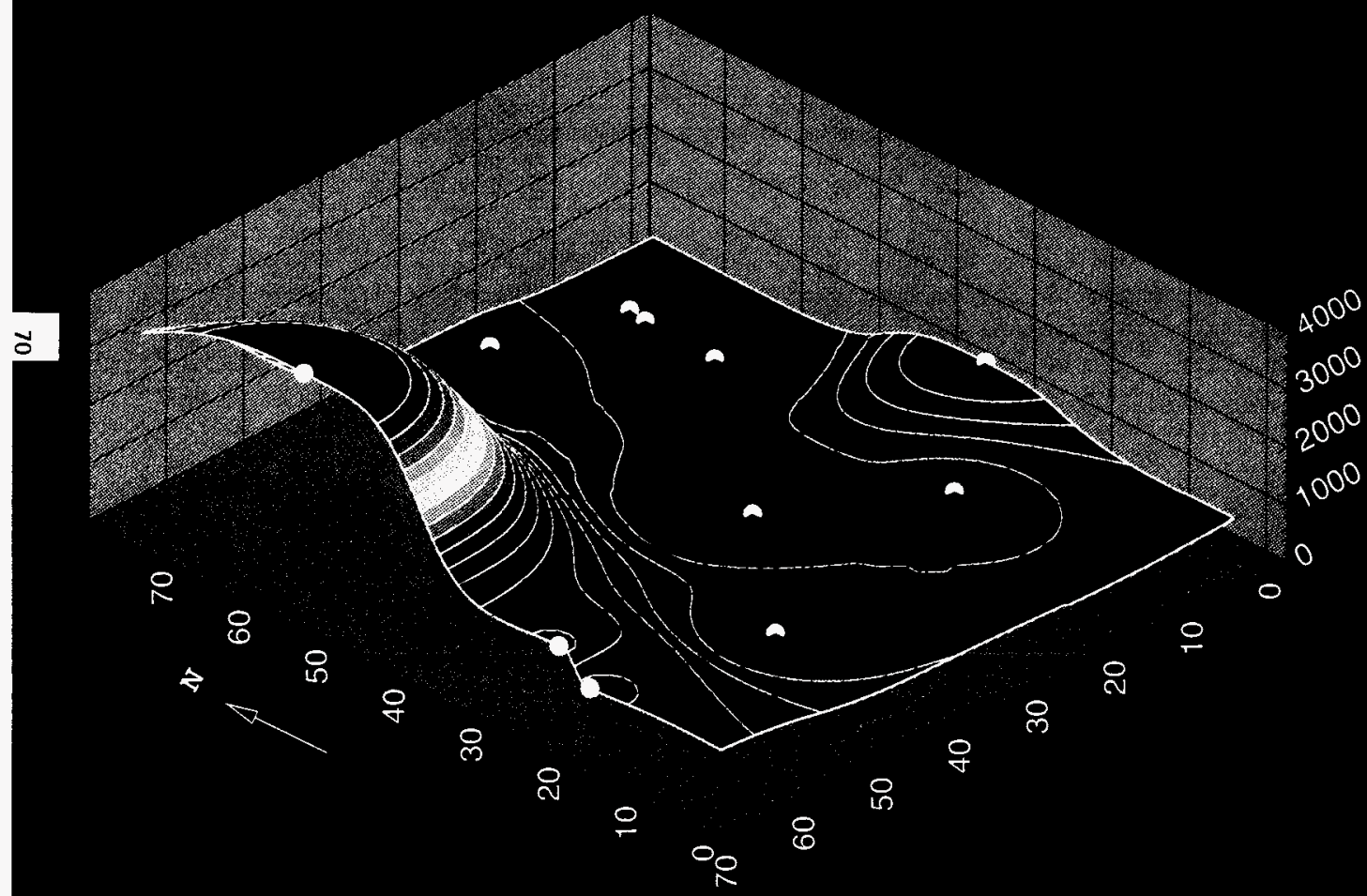


WHC-SD-SNF-TI-038, Rev. 0

APPENDIX B

VISUALIZATION PLOTS OF FLOOR SLUDGE DEPTH DATA FROM $K$ EAST 
WHC-SD-SNF-TI-038, Rev. 0

This page intentionally left blank. 


\section{K-E BASIN SLUDGE DEPTH MAP}

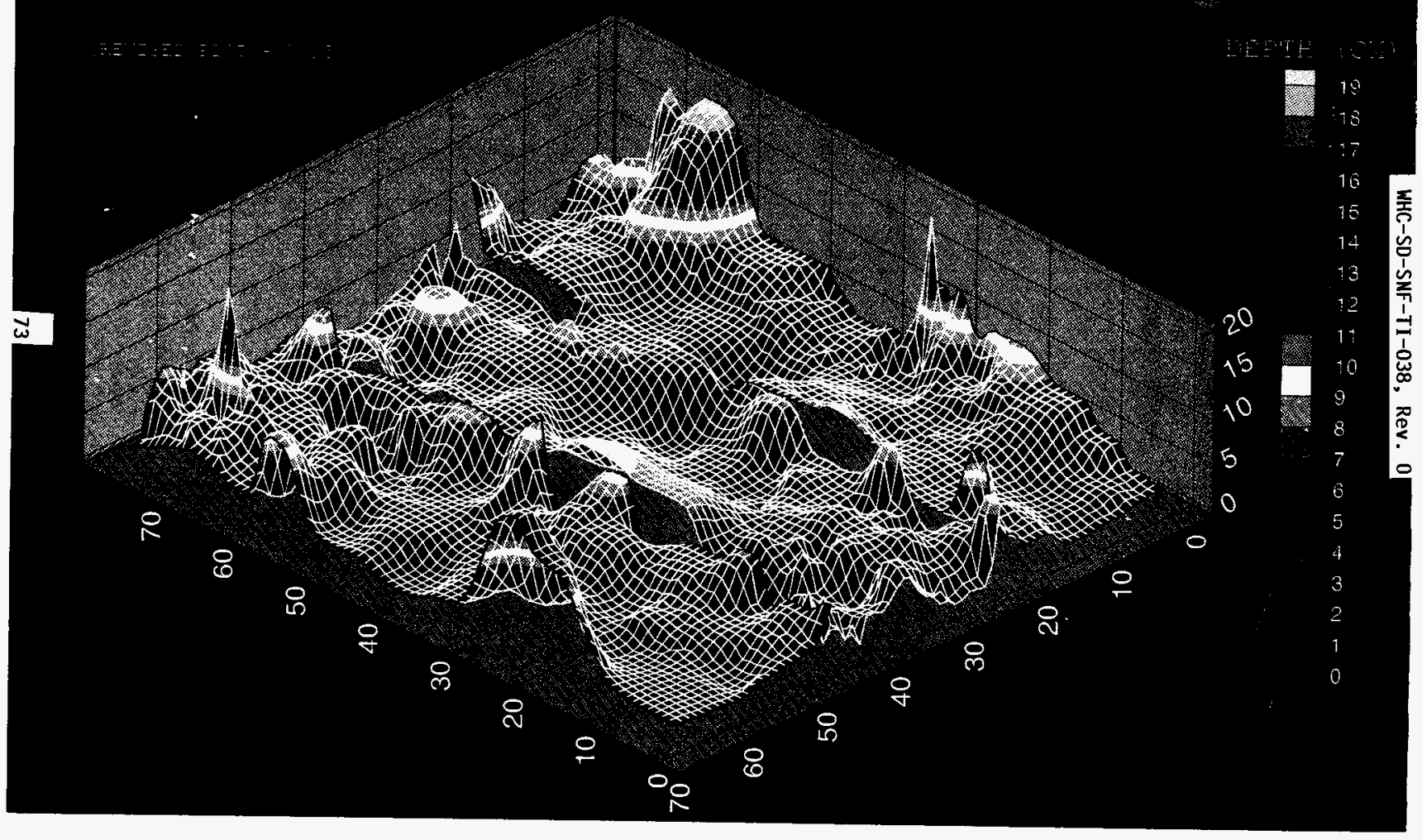


WHC-SD-SNF-TI-038, Rev. 0

This page intentionally left blank. 
WHC-SD-SNF-TI-038, Rev. 0

APPENDIX C

VISUALIZATION PLOTS OF MASS DISTRIBUTIONS OF SELECTED CONSTITUENTS FOR $K$ EAST FLOOR SLUDGE 
WHC-SD-SNF-TI-038, Rev. 0

This page intentionally left blank. 


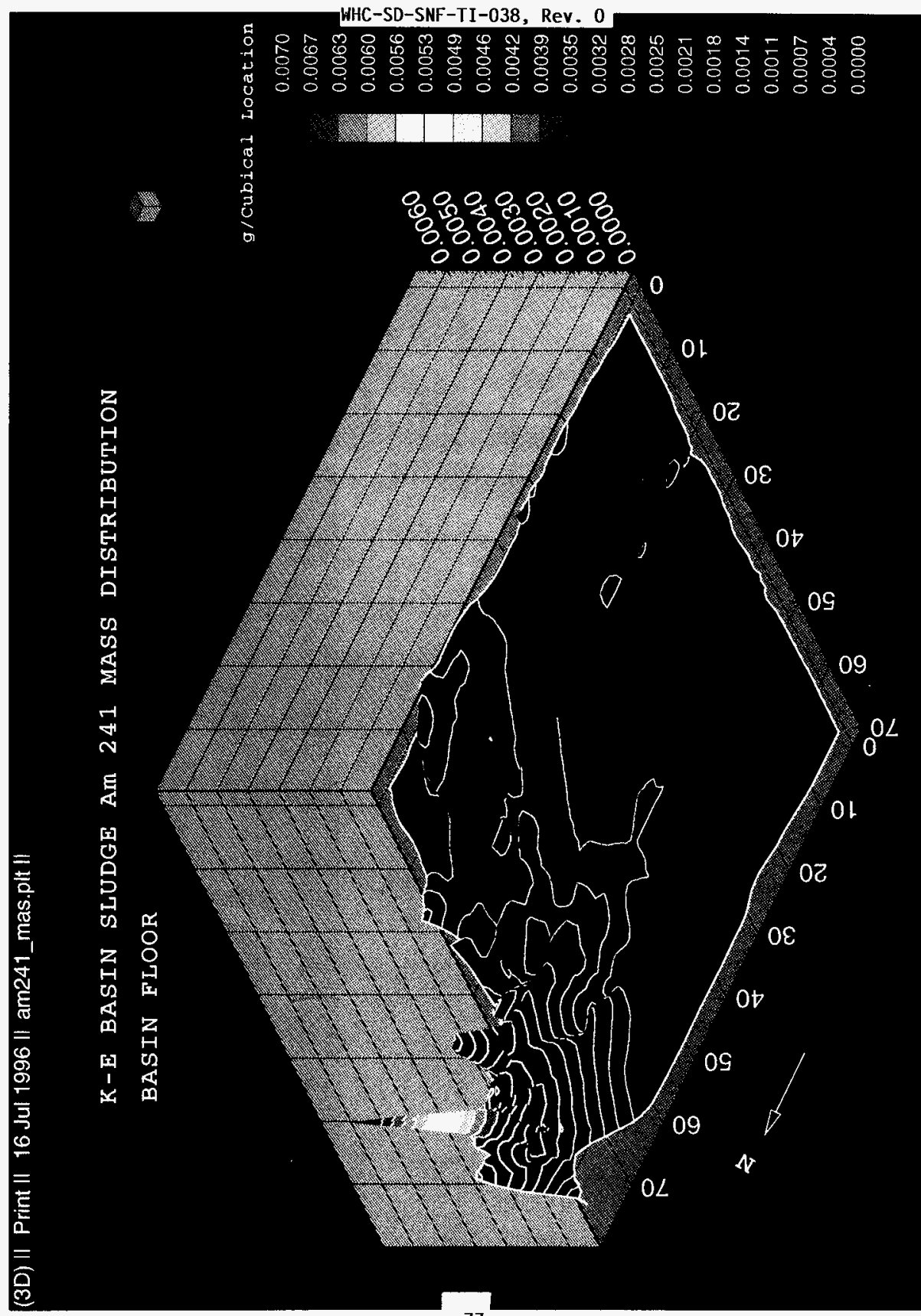




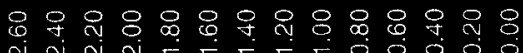

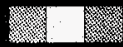

8.8 .8 .8 .9 .8 .8 .8

-

$$
\mathrm{A} \mathrm{d} \mathrm{C}<\mathrm{O} 0 \mathrm{O}
$$

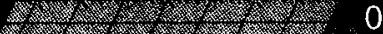

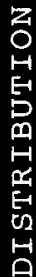

.

.

:
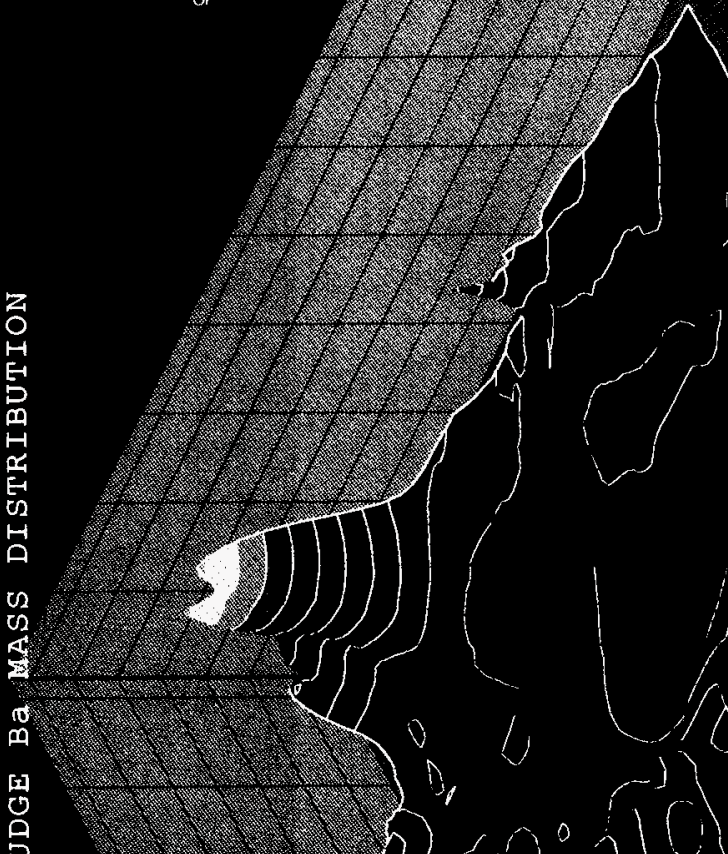

OL

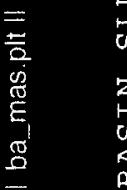

是

必

$z \mathrm{O}$
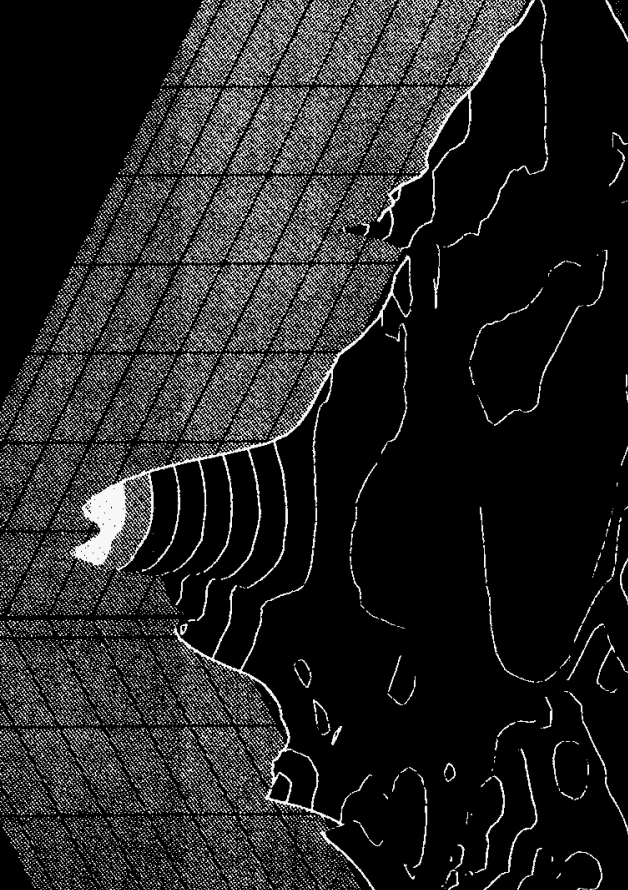

藏

m

m $\underset{H}{Z}$

积

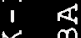
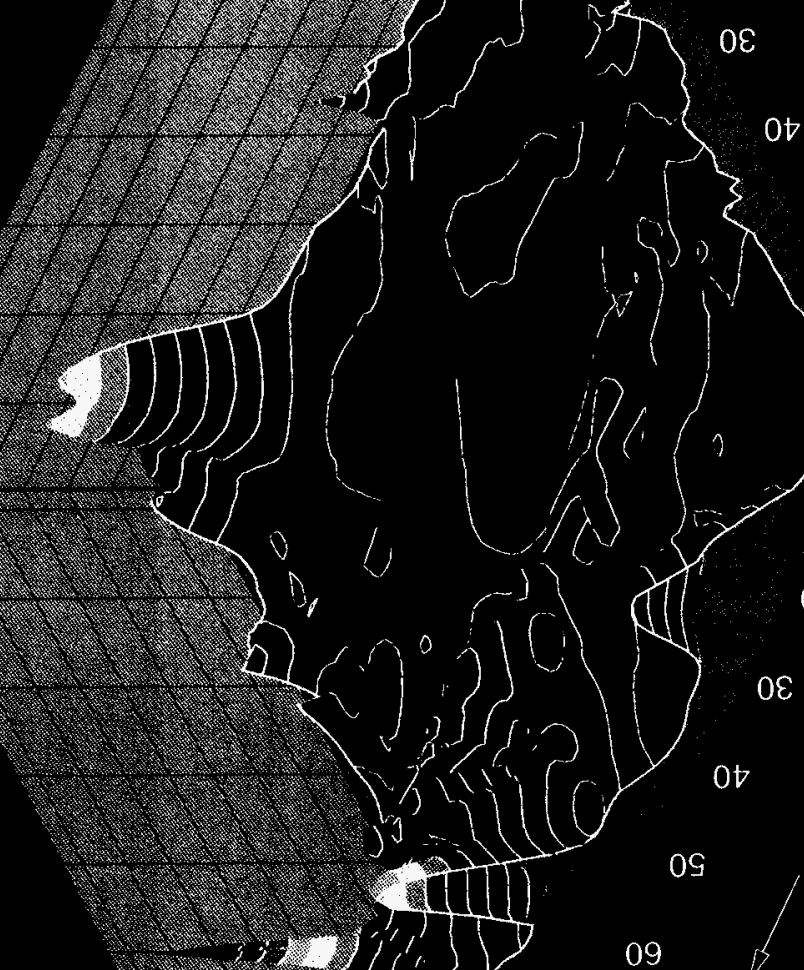

$0 z$
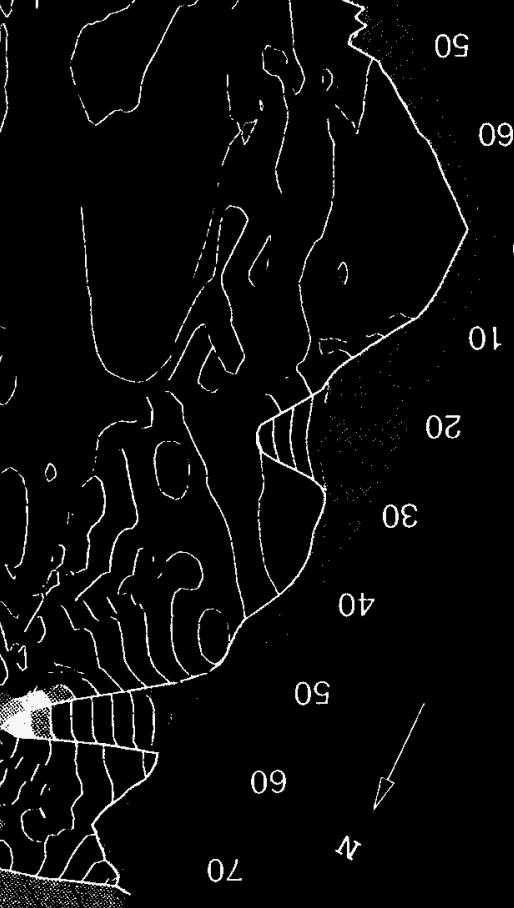

09

ot

OE 
WHC-SD-SNF-TI-038, Rev. 0

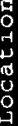

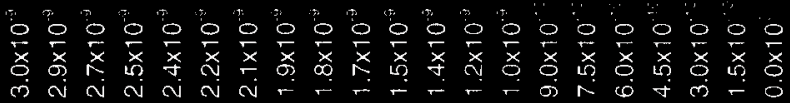

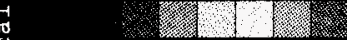

1)

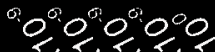

$$
\begin{aligned}
& \text { tot to.to. } \\
& \Delta \text { त } \omega^{\circ}<0
\end{aligned}
$$

2

은

N

N

m

回

○

ค

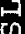

○

$H$

U2 [1

夰

a

(19) os

1
1

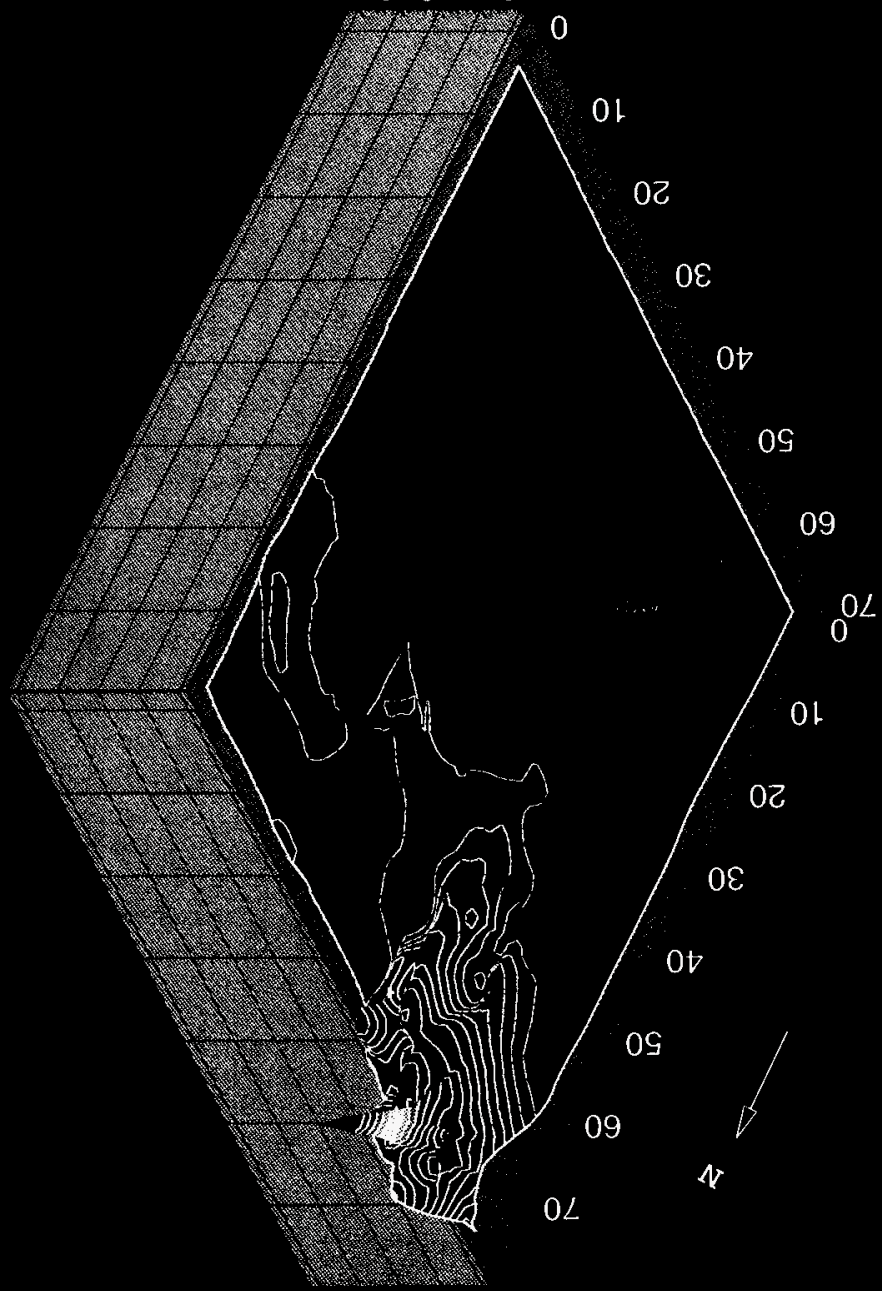


HHC-SD-SNF-TI-038, Rev. 0

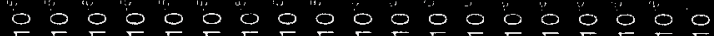

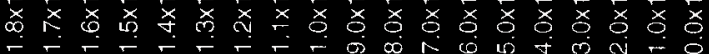

X

$Z$
0
$H$
$E-1$
0
0
$H$
0
$E$
0
$H$
$日$
0
0
0
4

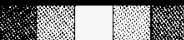

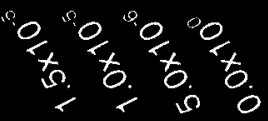

0

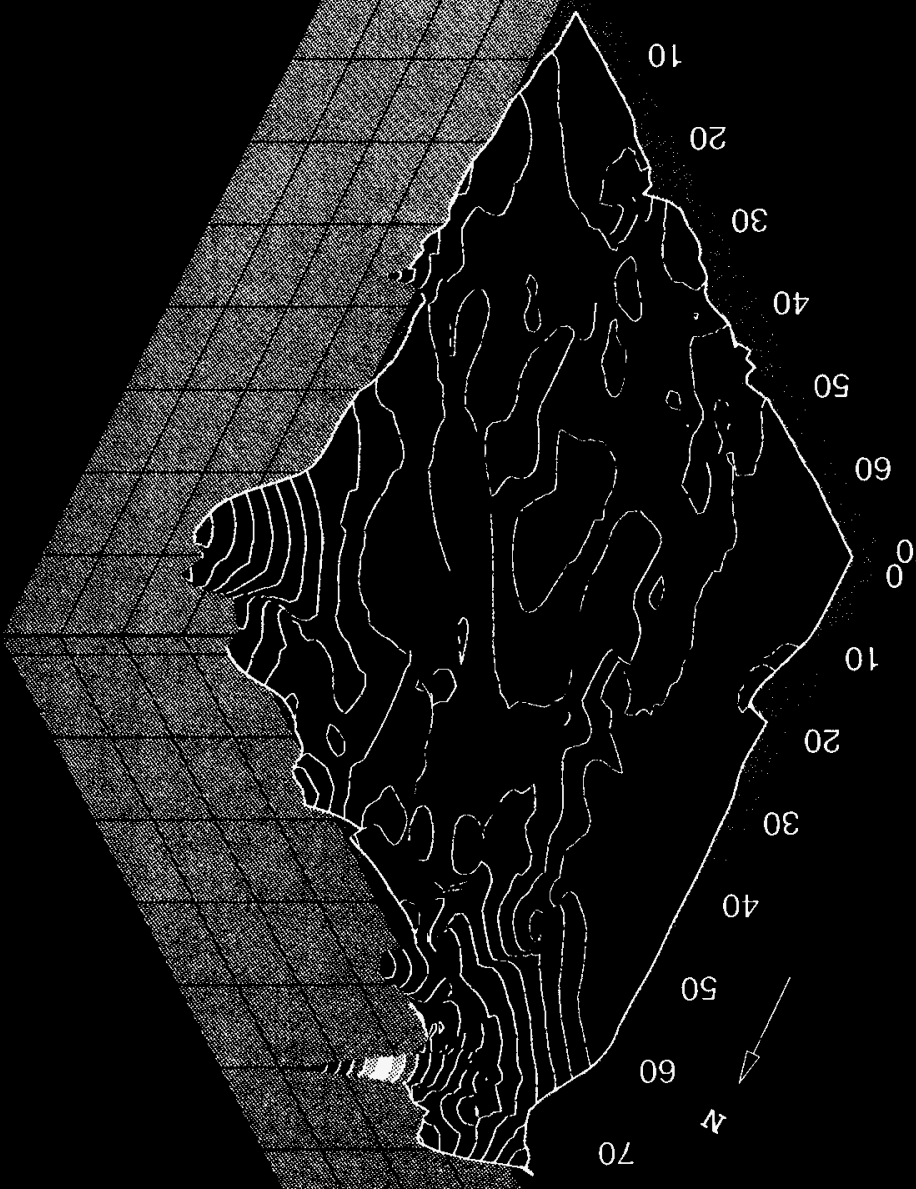

0

H

$\sum^{2}$

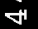

$\ddot{0}$

$\underline{\underline{\alpha}}$

g

ᄋ

占

Z

O

m 


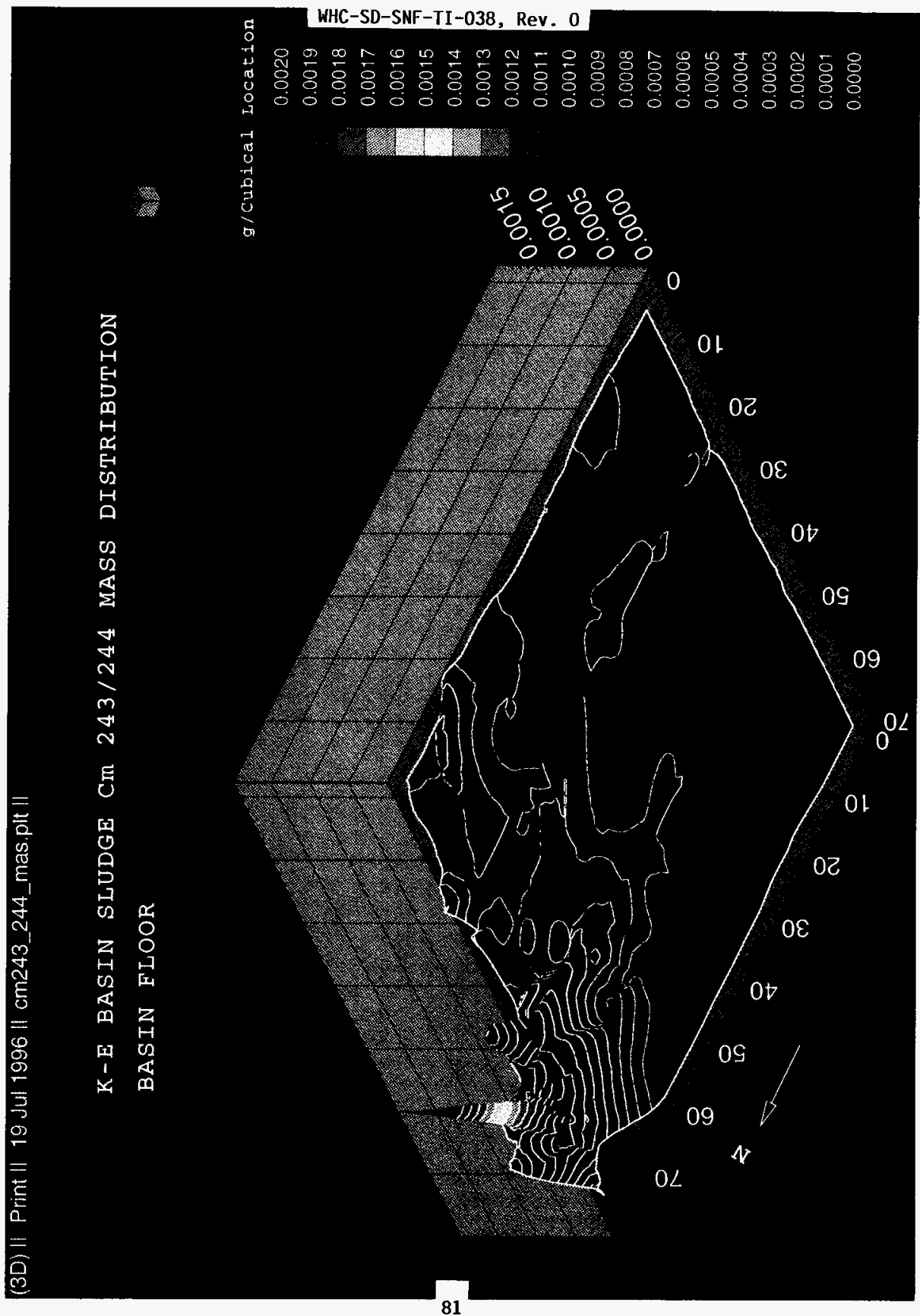


WHC-SD-SNF-TI-038, Rev. 0

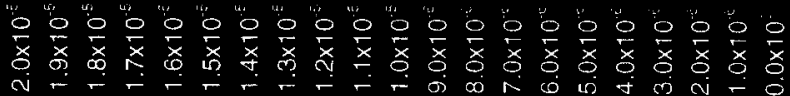

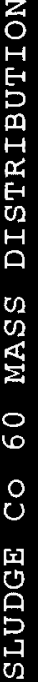

짔

0.000
$0.5+0.0$
$0<<00$

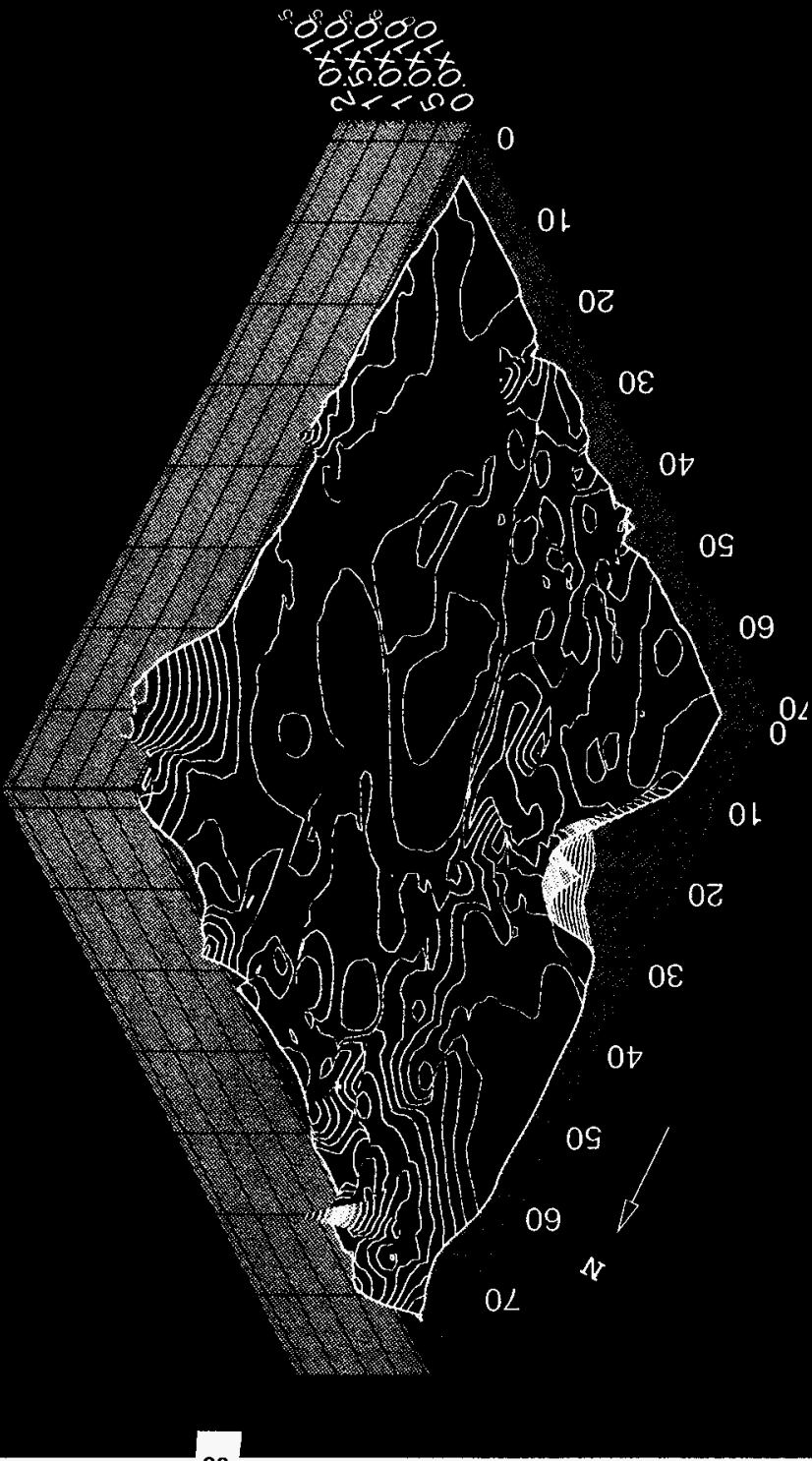

0

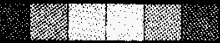


WHC-SD-SNF-TI-038, Rev. 0

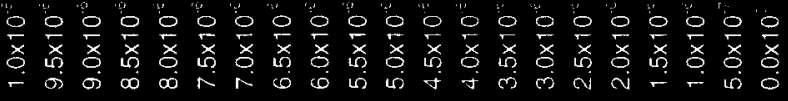

W

$$
\begin{aligned}
& 0^{\circ} 0^{\circ} 0^{\circ} 0 \\
& \text { t }{ }^{2}+t^{2} t^{2}+0 .
\end{aligned}
$$

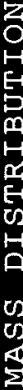

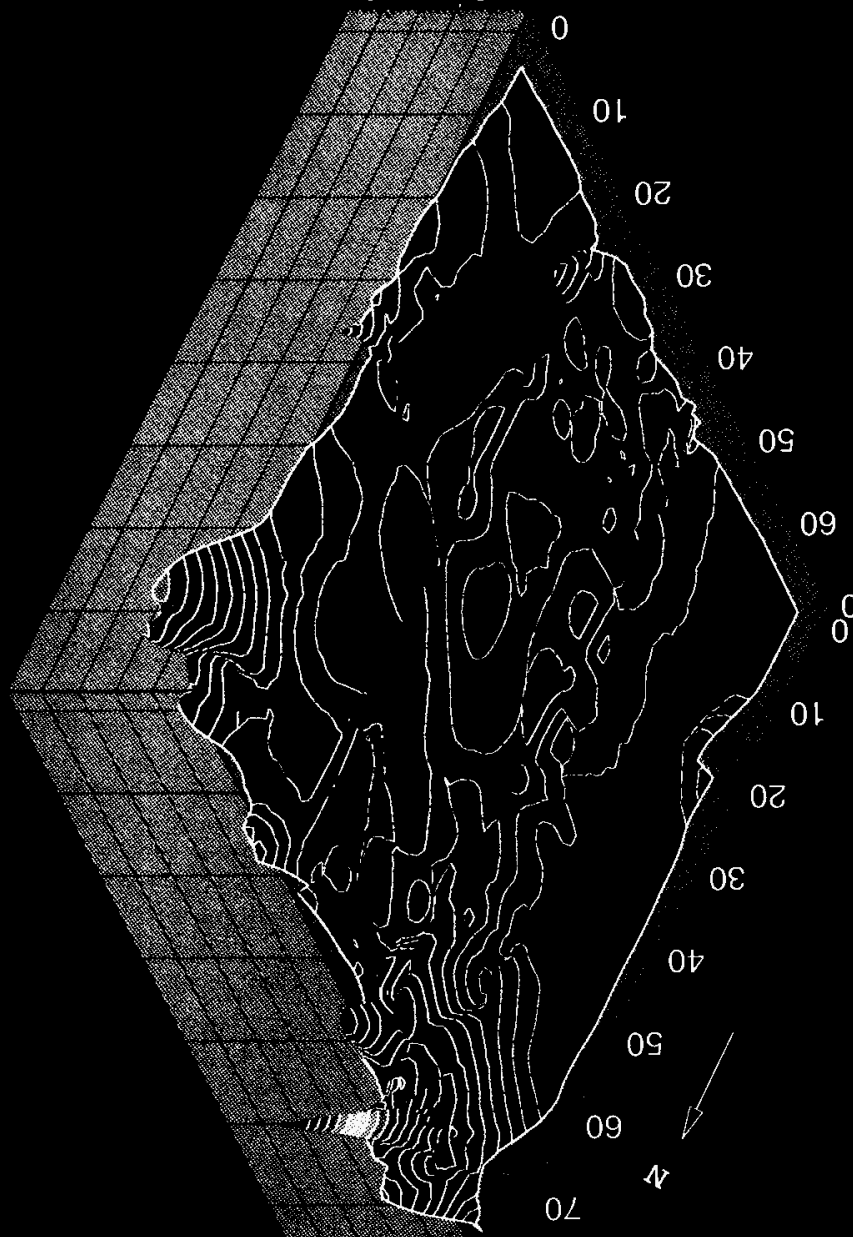

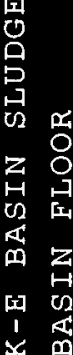


WHC-SD-SNF-TI-038, Rev. 0

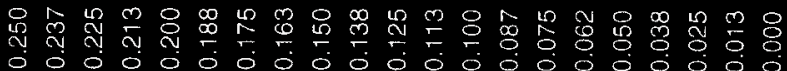

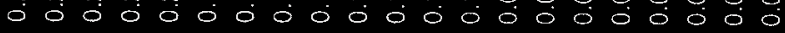

3.

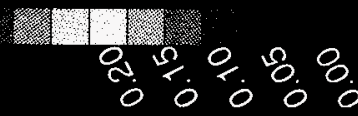

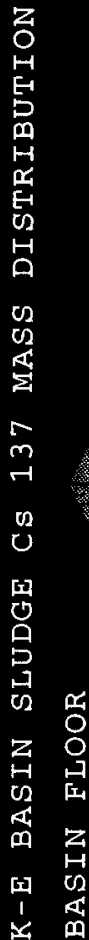

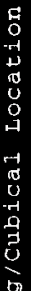
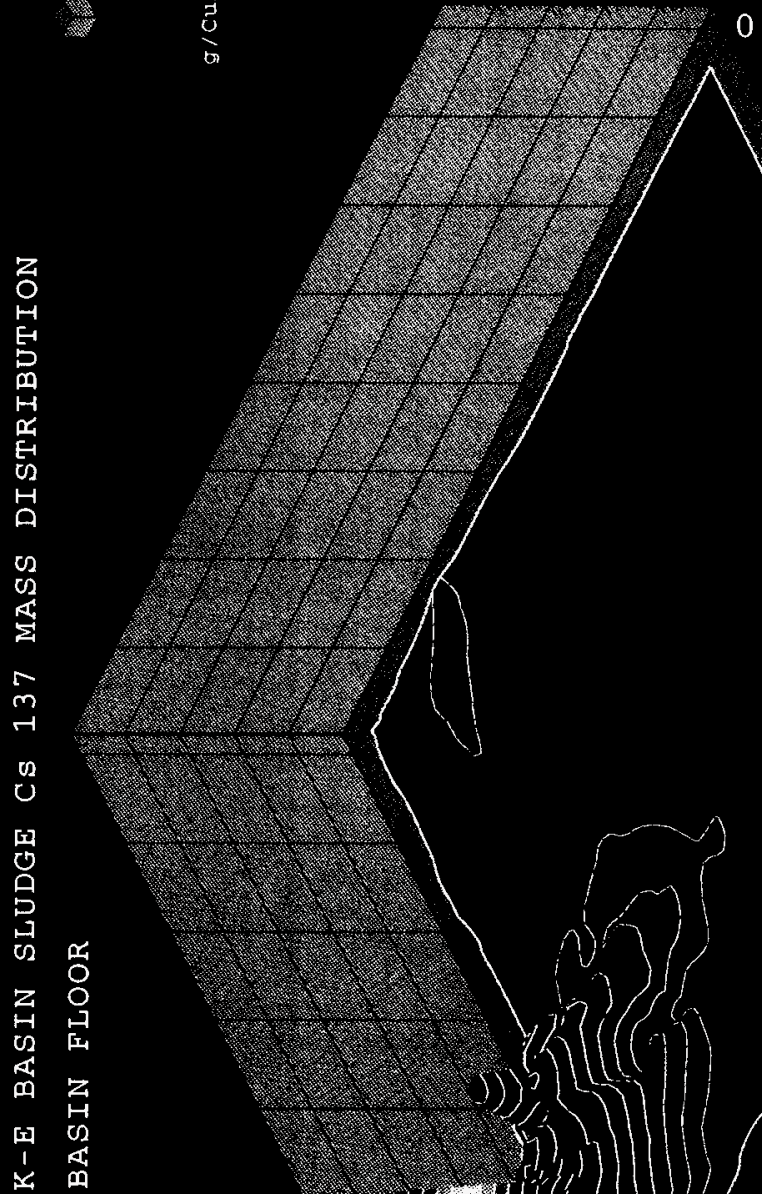

ot
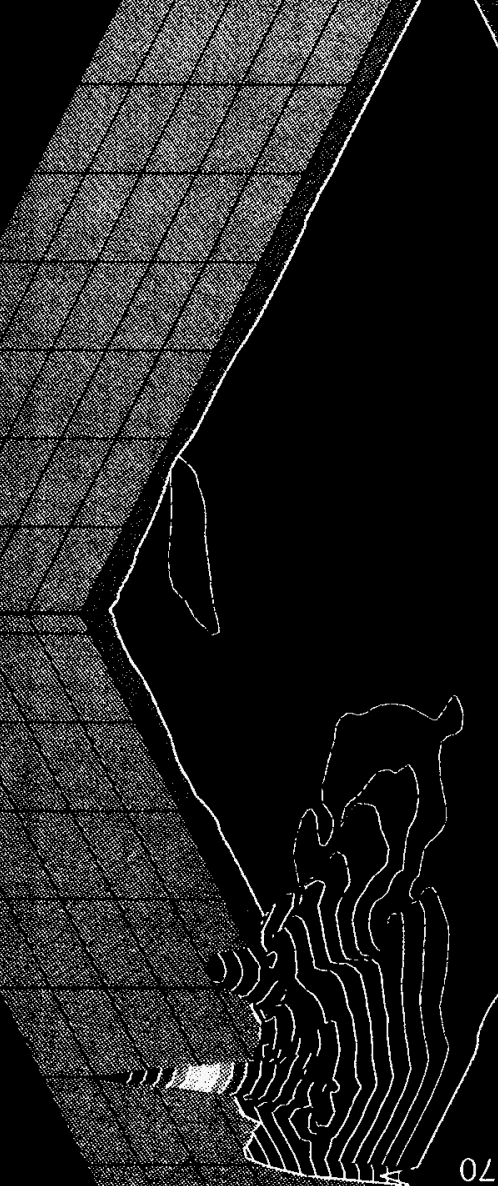

$0 L$
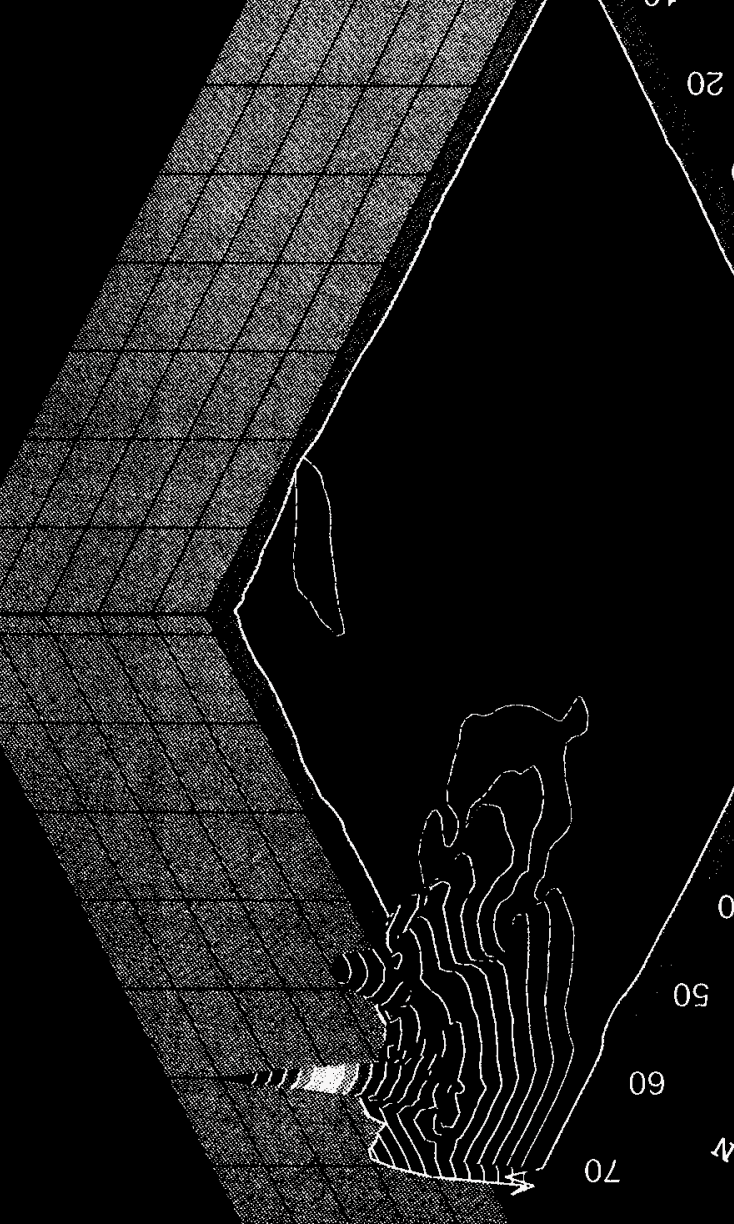

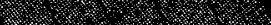

09

09

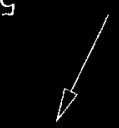

$\checkmark$ 


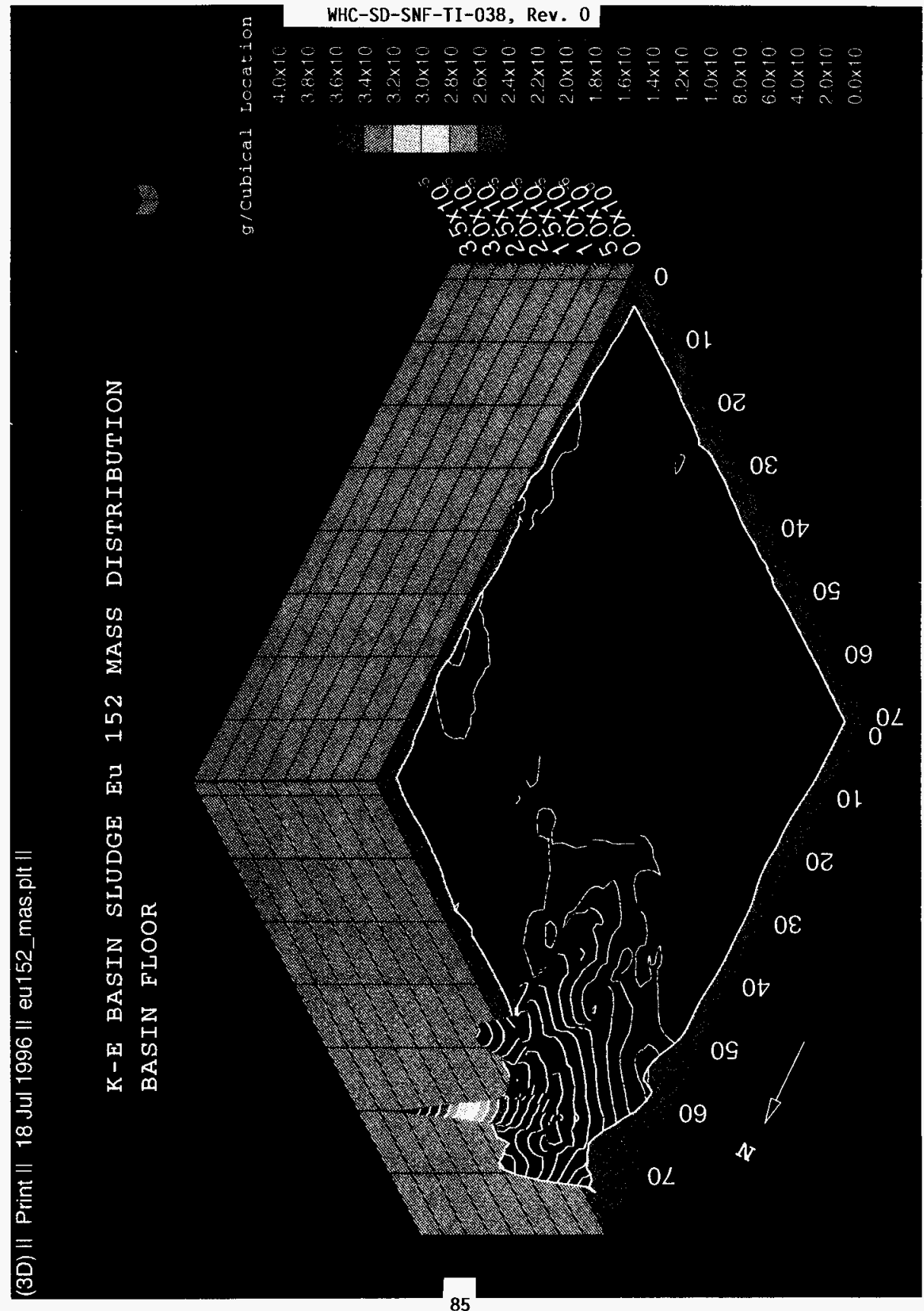


WHC-SD-SNF-TI-038, Rev. 0

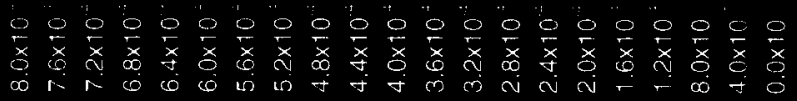

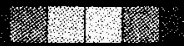

w

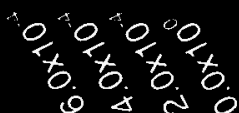

号

EN

m

$\mapsto$

E-1

U)

봄

c)

4

바

n

3

[a]

$=\frac{\varrho}{\rho}$

要

때

z $\mathrm{O}$

盛

西

m $z$

回 $\mathrm{H}$

14

䑻

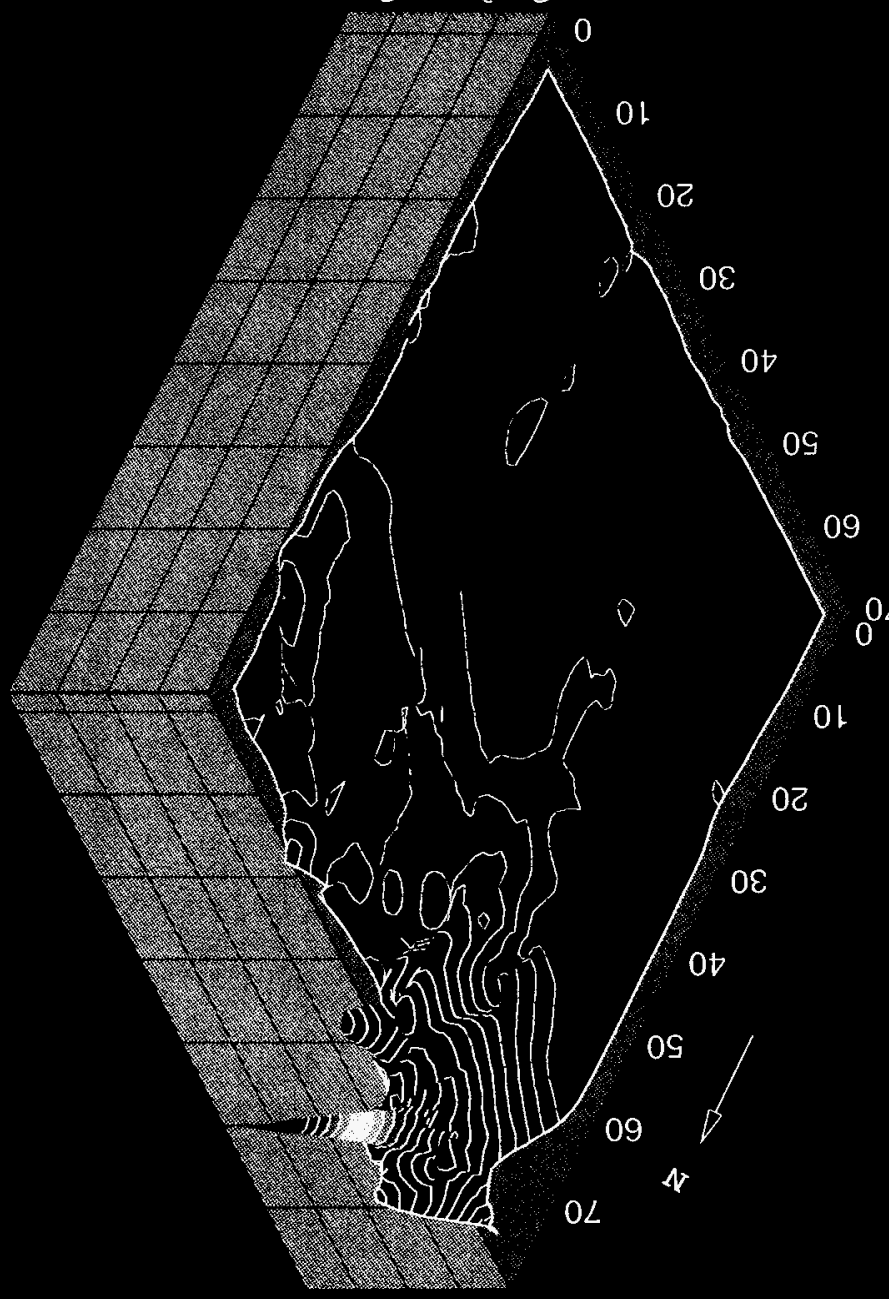

$\frac{7}{9}$ 
WHC-SD-SNF-TI-038, Rev. 0.

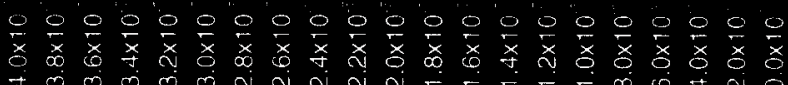
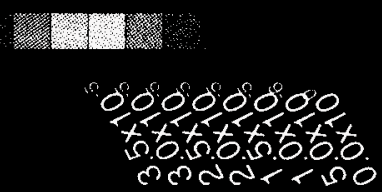

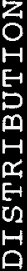

0

U

in

in

นก

고

\%

0
0
0
-1
0
7
0
0
0

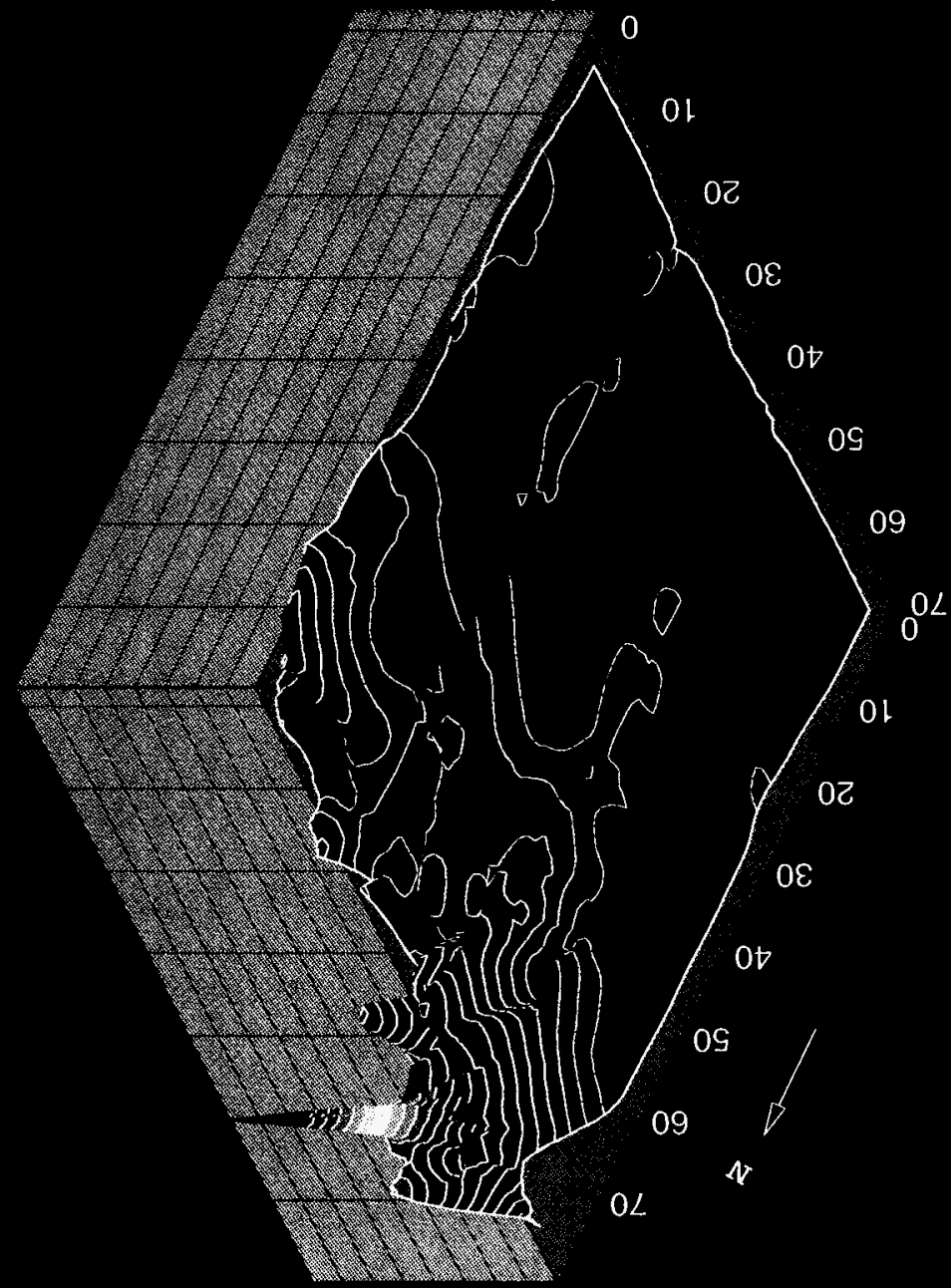

बิ

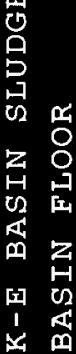


HHC-SD-SNF-TI-038, Rev - 0

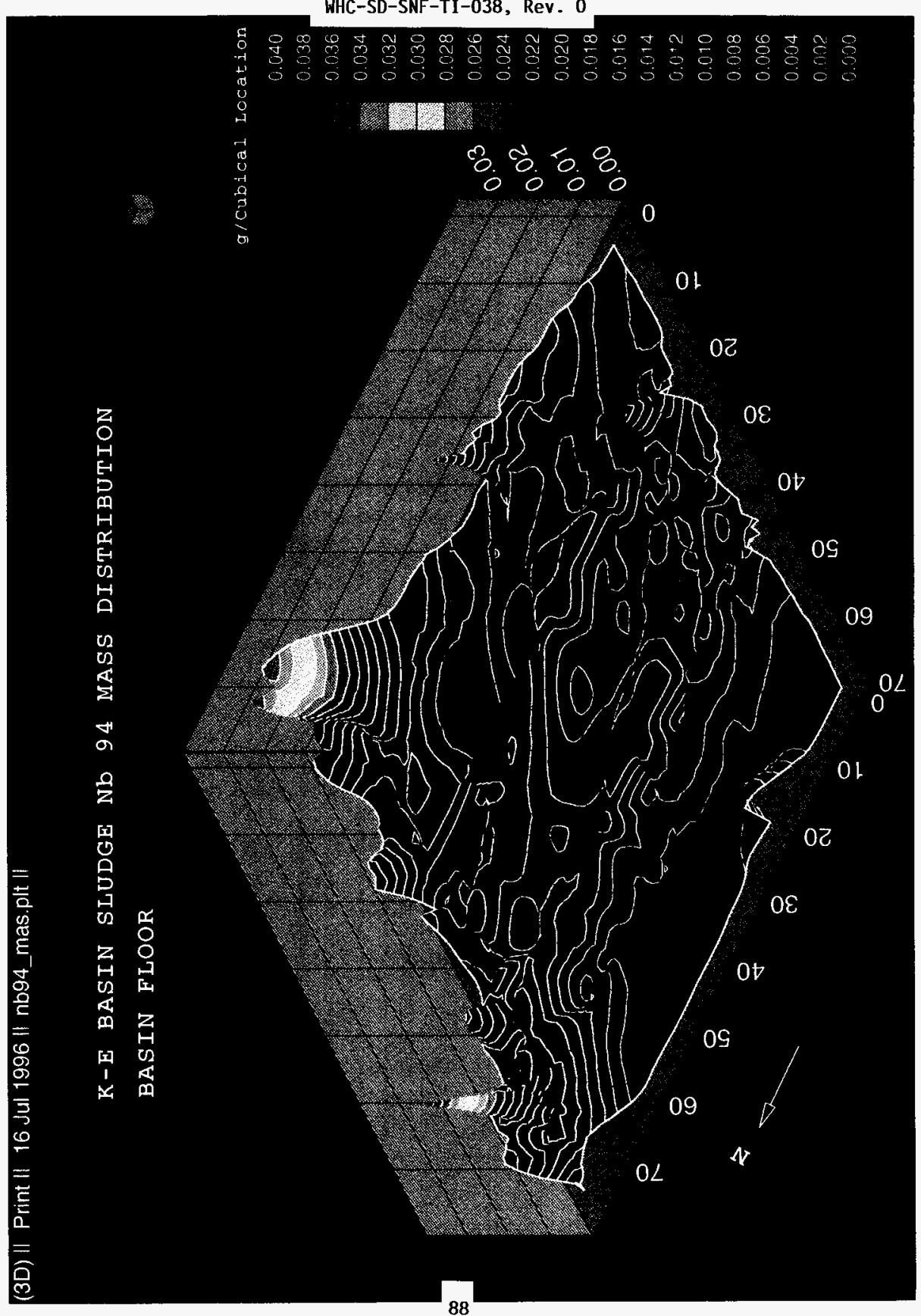


WHC-SD-SNF-TI-038, Rev. 0

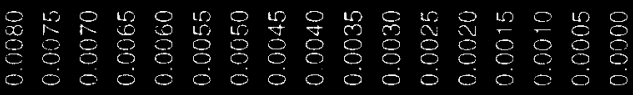

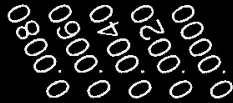

$Z$
0
$H$
$E-1$
0
0
$H$
0
$E$
0
$\stackrel{1}{0}$
0
0
0
0

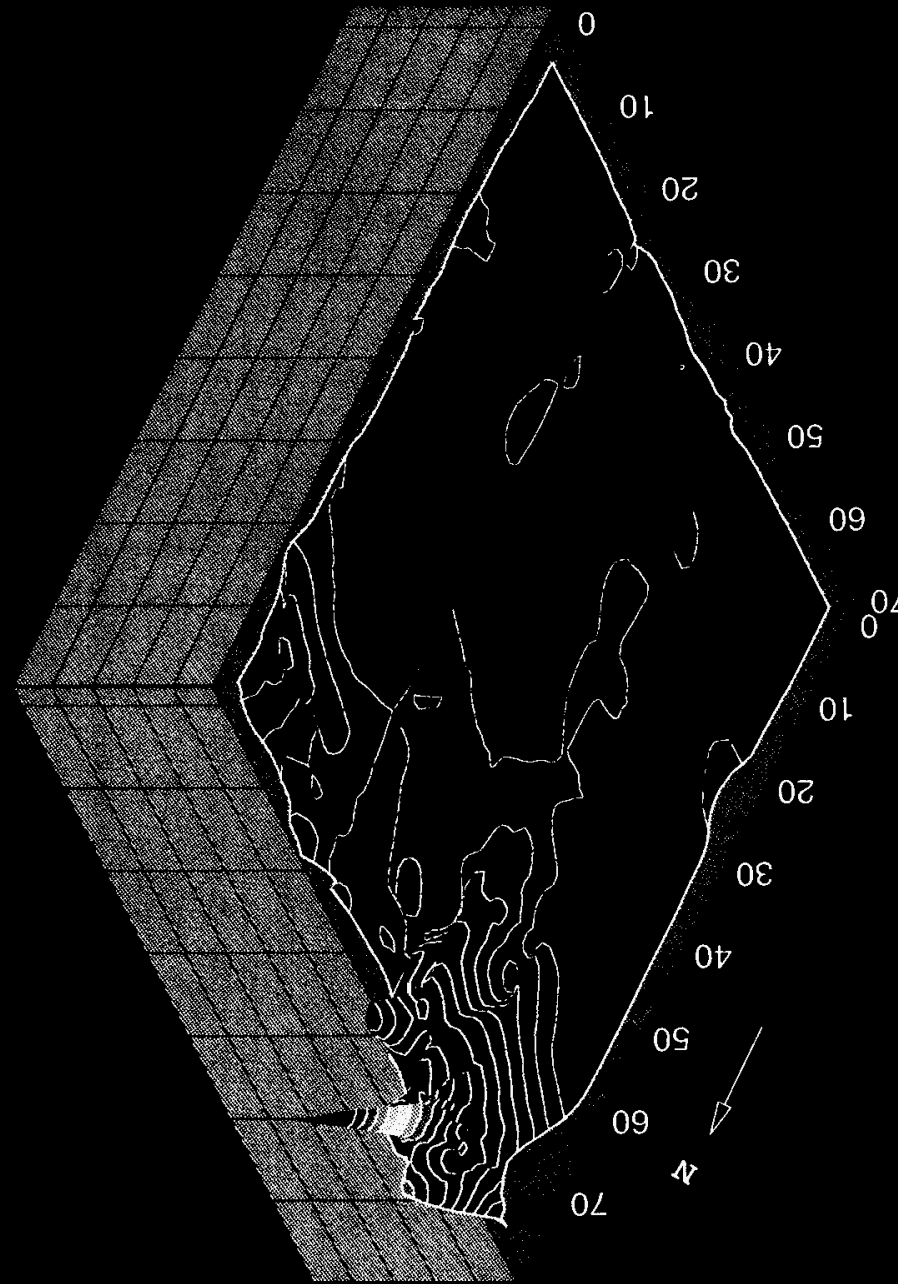


WHC-SD-SNF-TI-038, Rev. 0

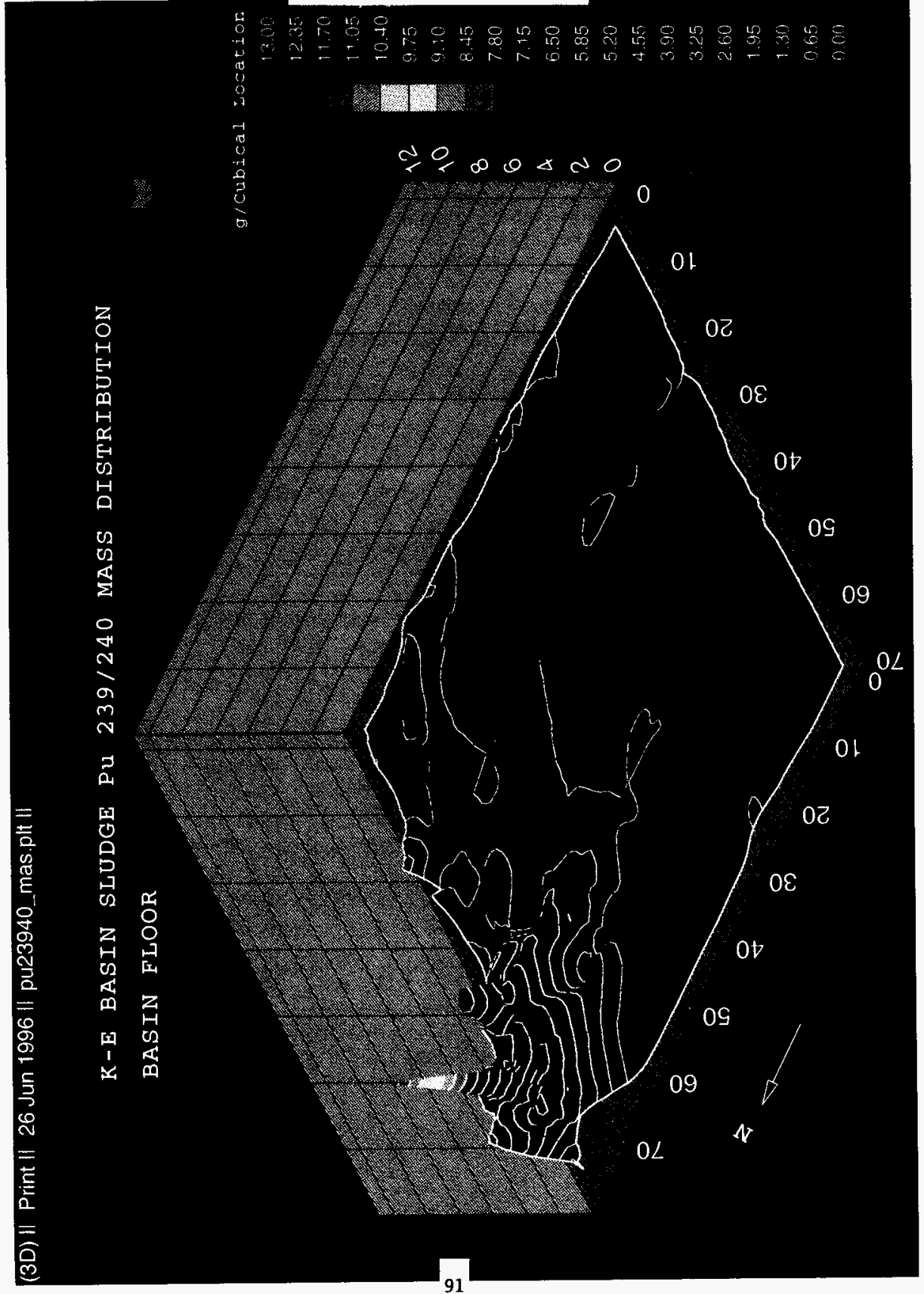


WHC-SD-SNF-TI-038, Rev. 0

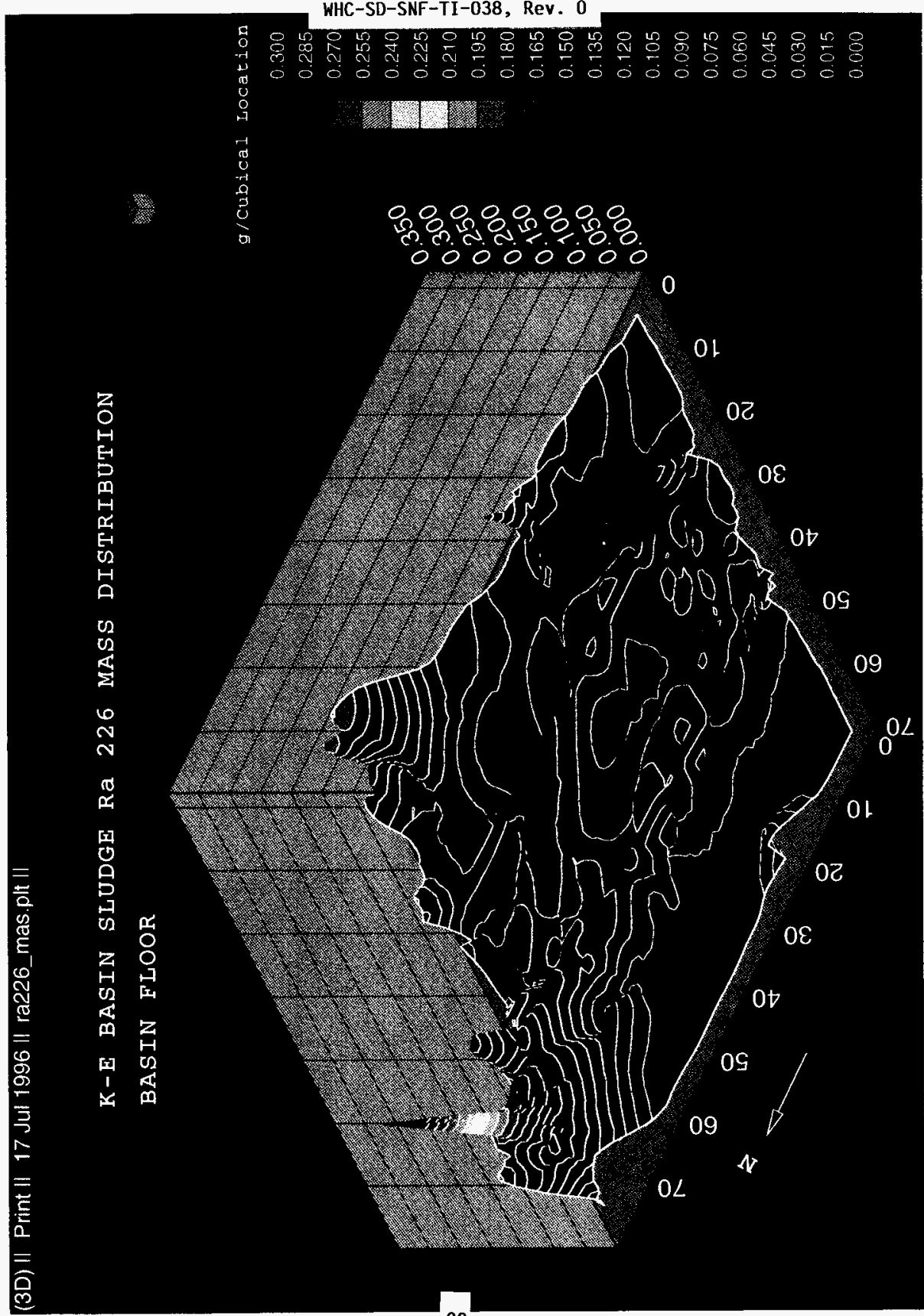


WHC-SD-SNF-TI-038, Rev. 0

$B$
0

$\circ \circ 000000000000000000$

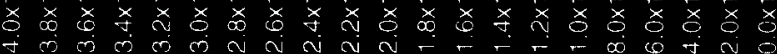

\%

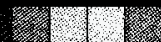

\section{Q00000000 to.to.to.toto. लीNतर 50}

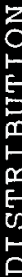

os

os

녹

它

$\not$

6

0

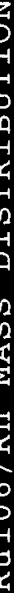

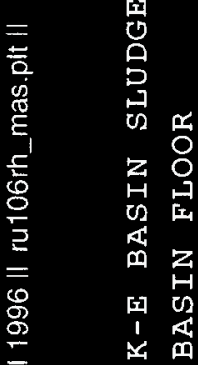

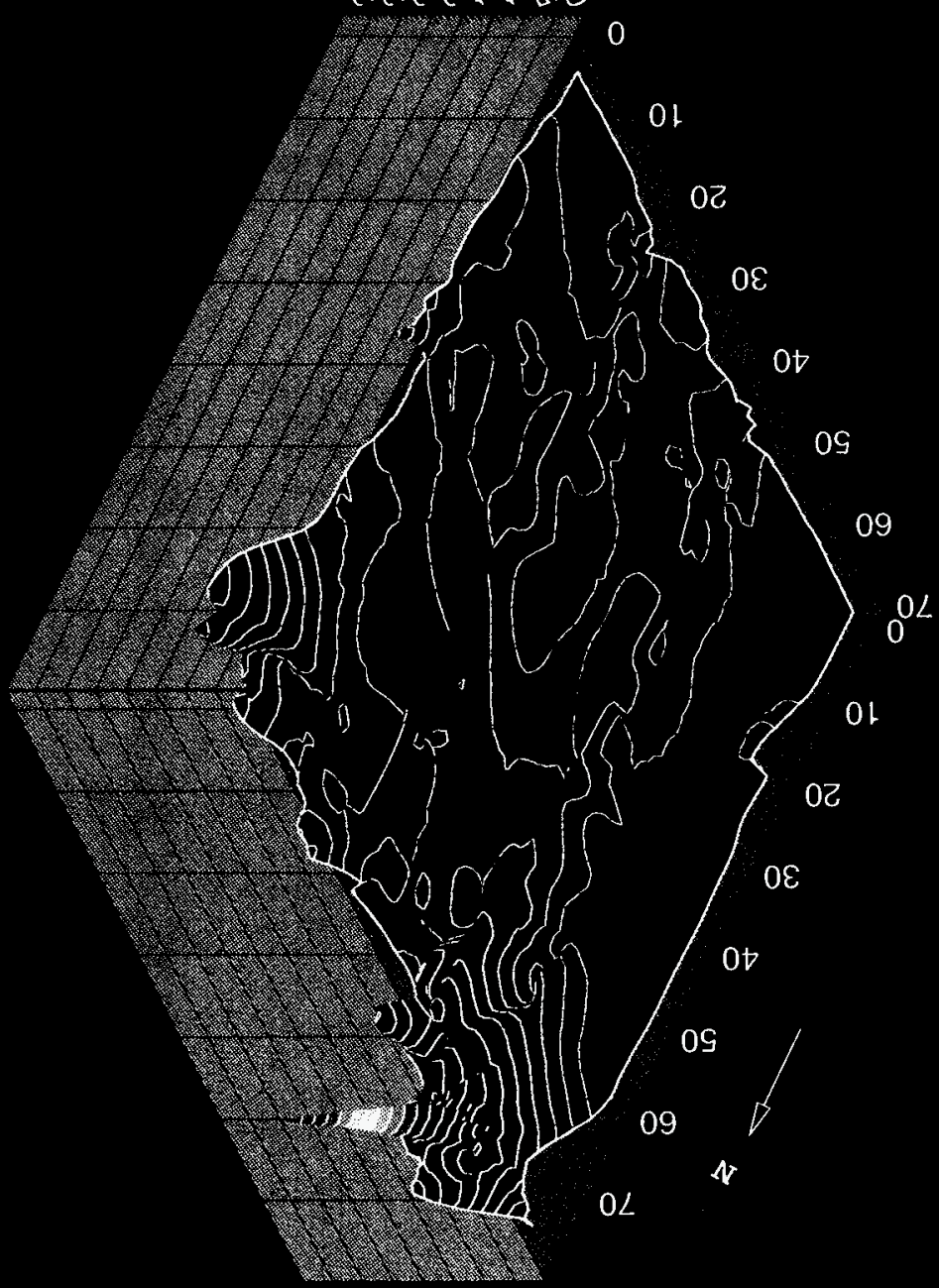


WHC-SD-SNF-TI-038, Rev . 0

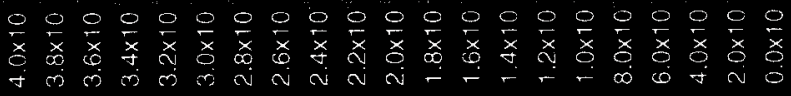

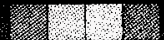

"00000000

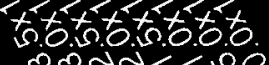

लnलतर 150

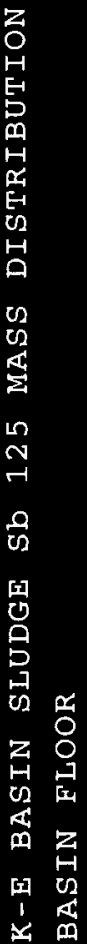

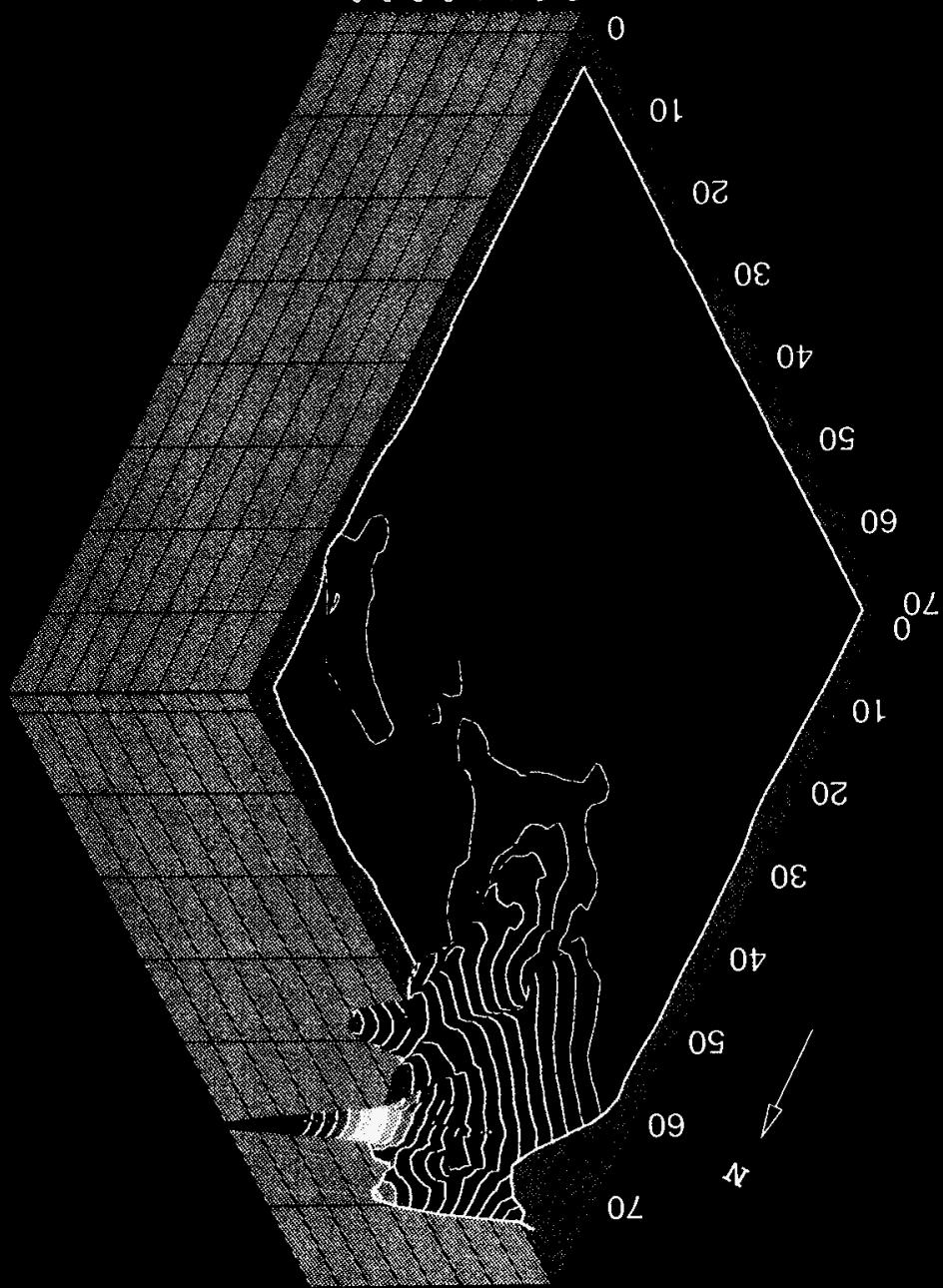


WHC-SD-SNF-TI-038, Rev. 0

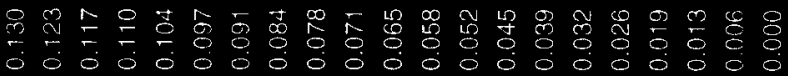

$$
\begin{aligned}
& \text { (4) } \\
& \frac{\pi}{0.008 .80 .80}
\end{aligned}
$$

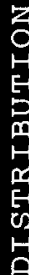

is

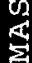

$\circ$

4

บ

回

ฮ

흥

盇

E $\quad z \quad$

음

के

$=$

$\infty$

8

$-\quad 1 \quad$

E $m$

$\frac{5}{3}$

$\infty$

N

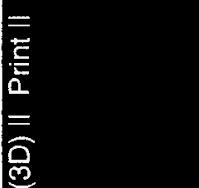

Ale 0

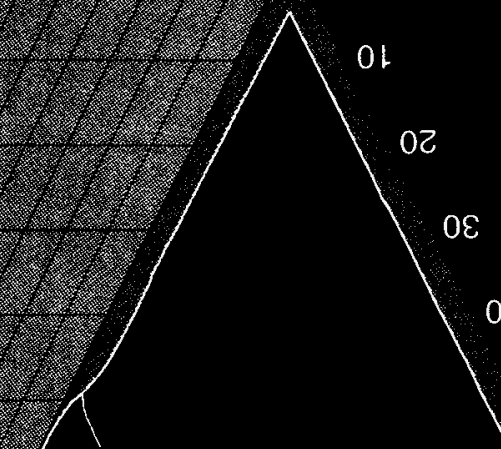

$0 S$

09

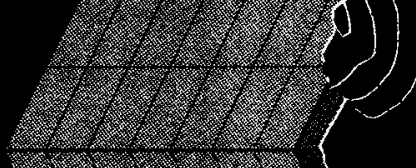


WHC-SD-SNF-TI-038, Rev. 0

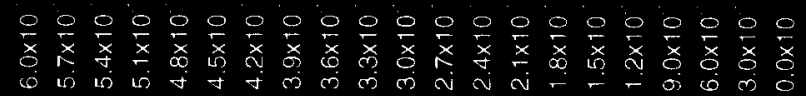

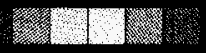

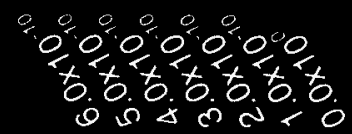

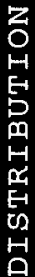

on

芯

$\infty$

○

E-1

红

号

$\alpha$

× 0

$H$

O 5

4

m

问 02

1
$\times$

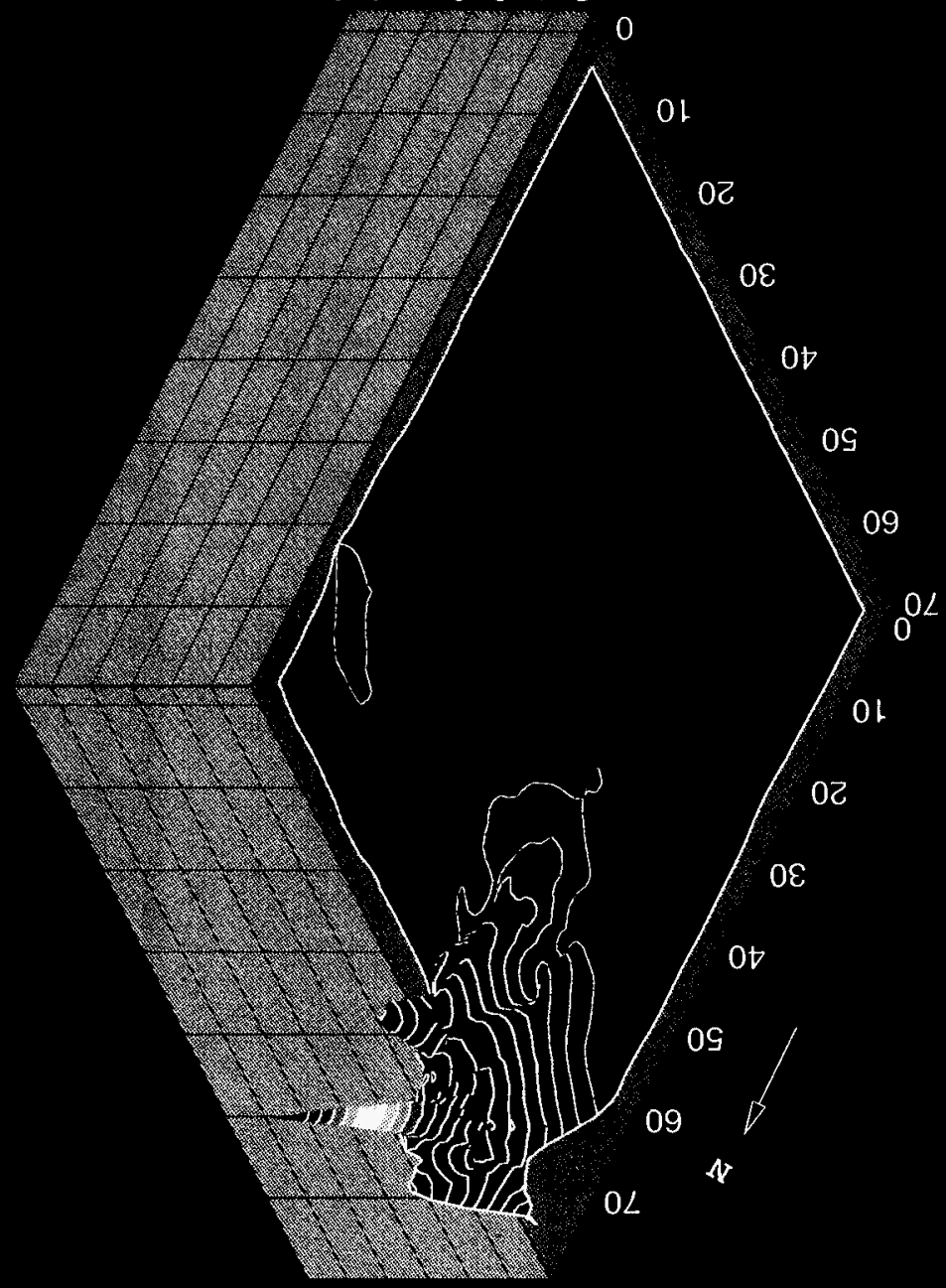

$\overline{\hat{0}}$ 
HHC-SD-SNF-TI-038, Rev. 0

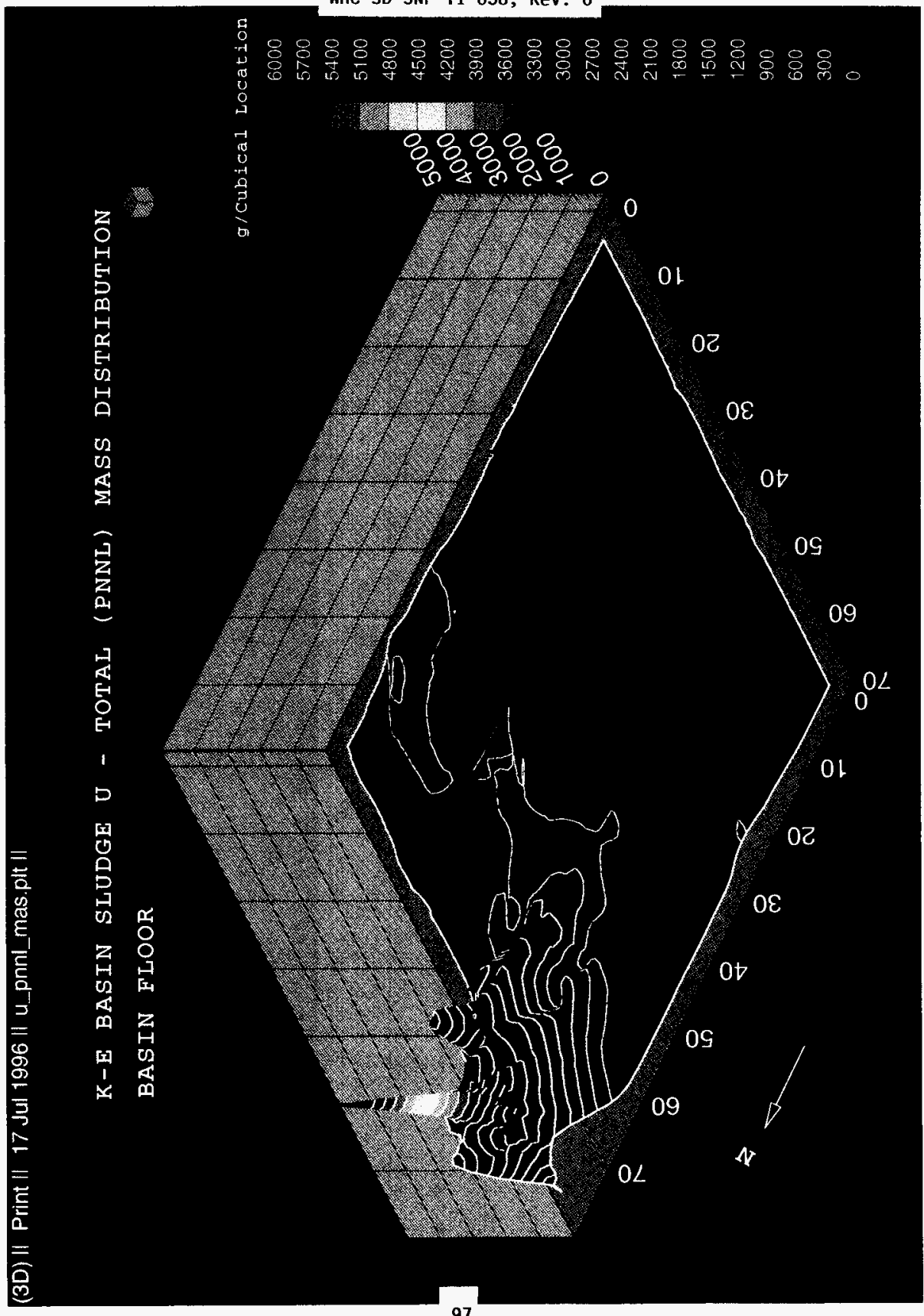


WHC-SD-SNF-TI-038, Rev. 0

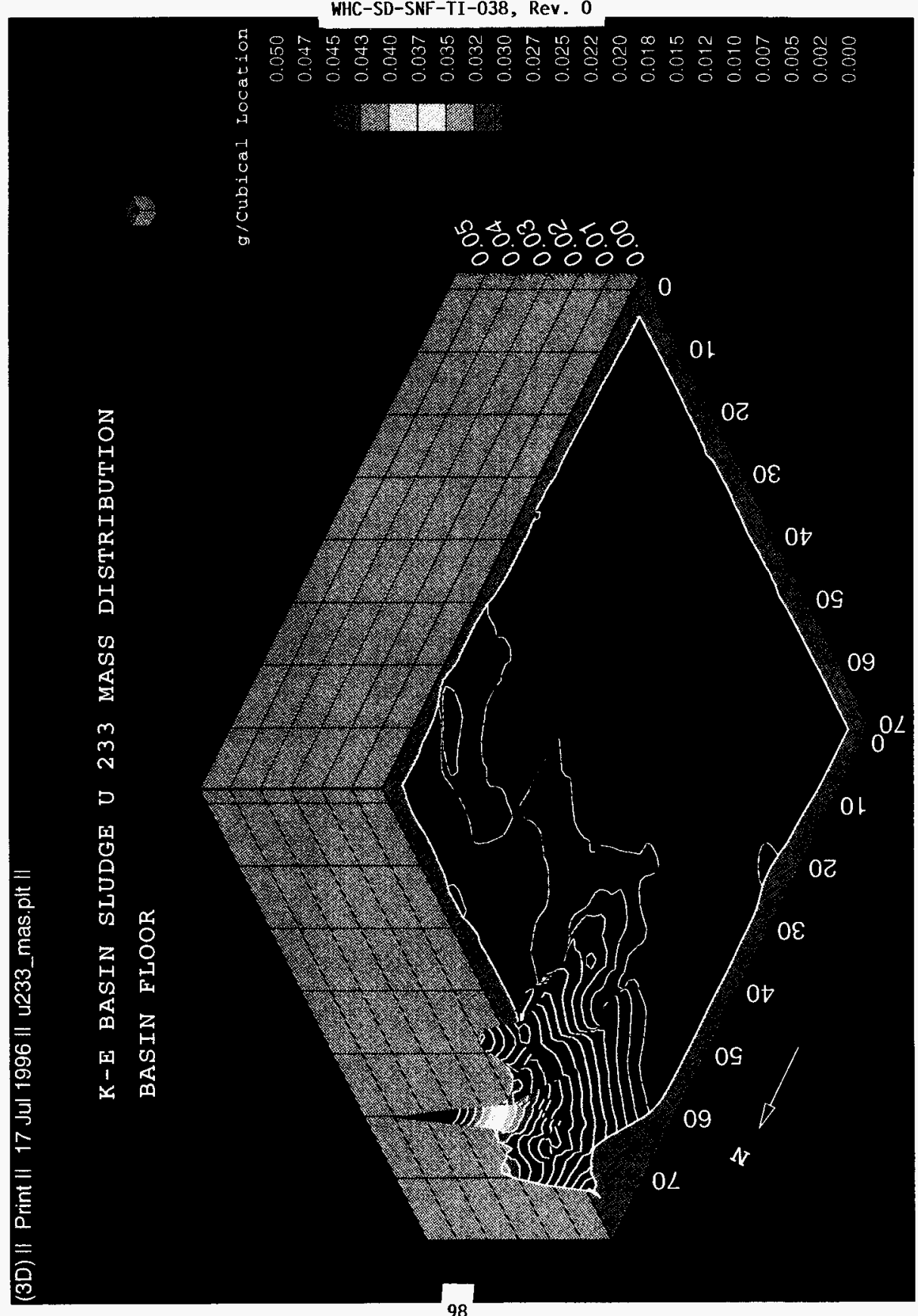


WHC-SD-SNF-TI-038, Rev. 0

$a$
0
-1
1
0
0
0
1
-1
0
0
$-r 1$
0
0
0
0
0

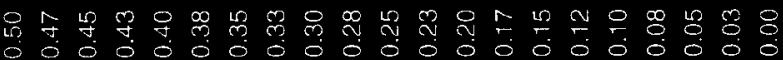

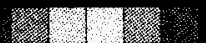

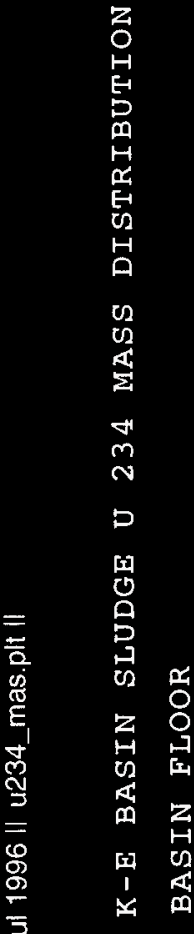

Q. Q 0.8

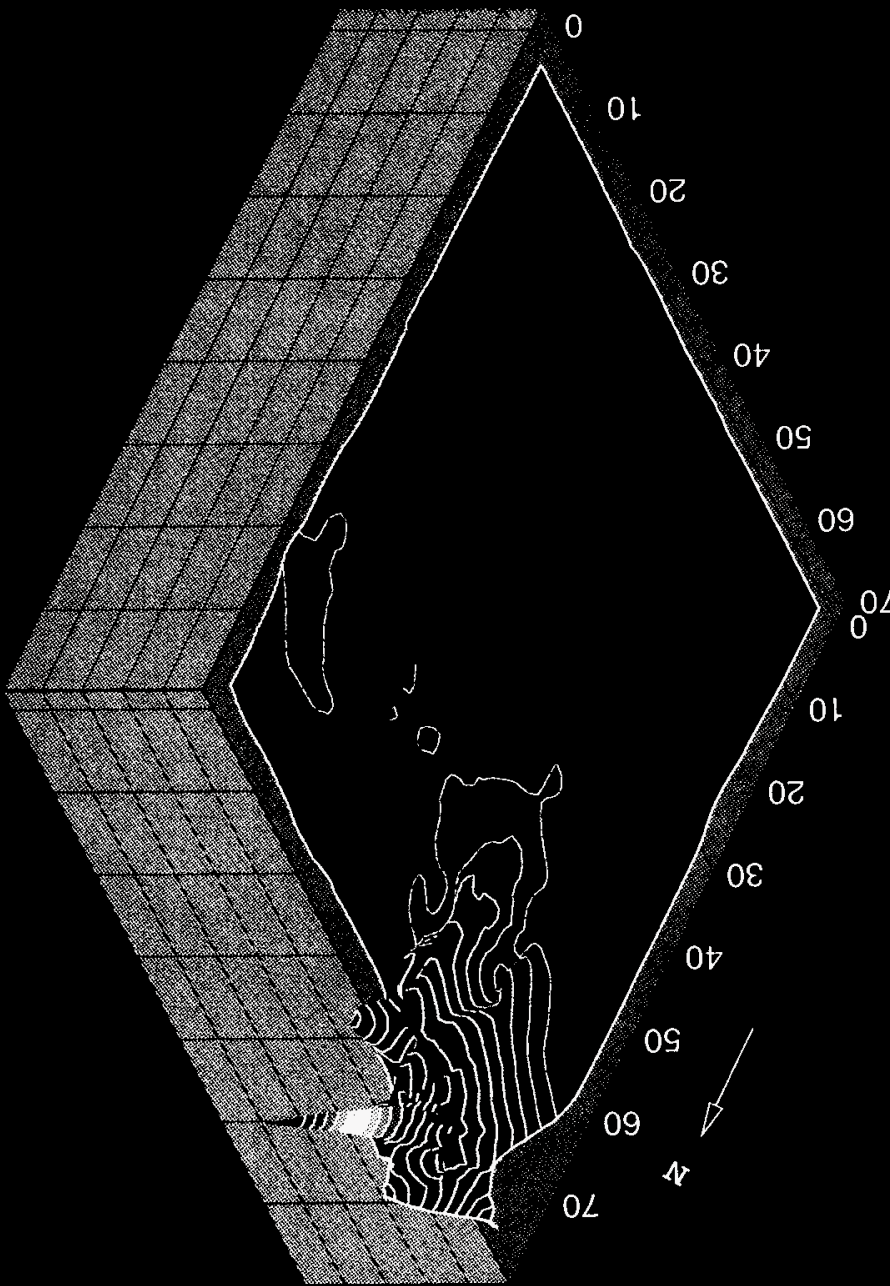


(3D) || Print || 26 Jun 1996 || u235_mas.plt ||

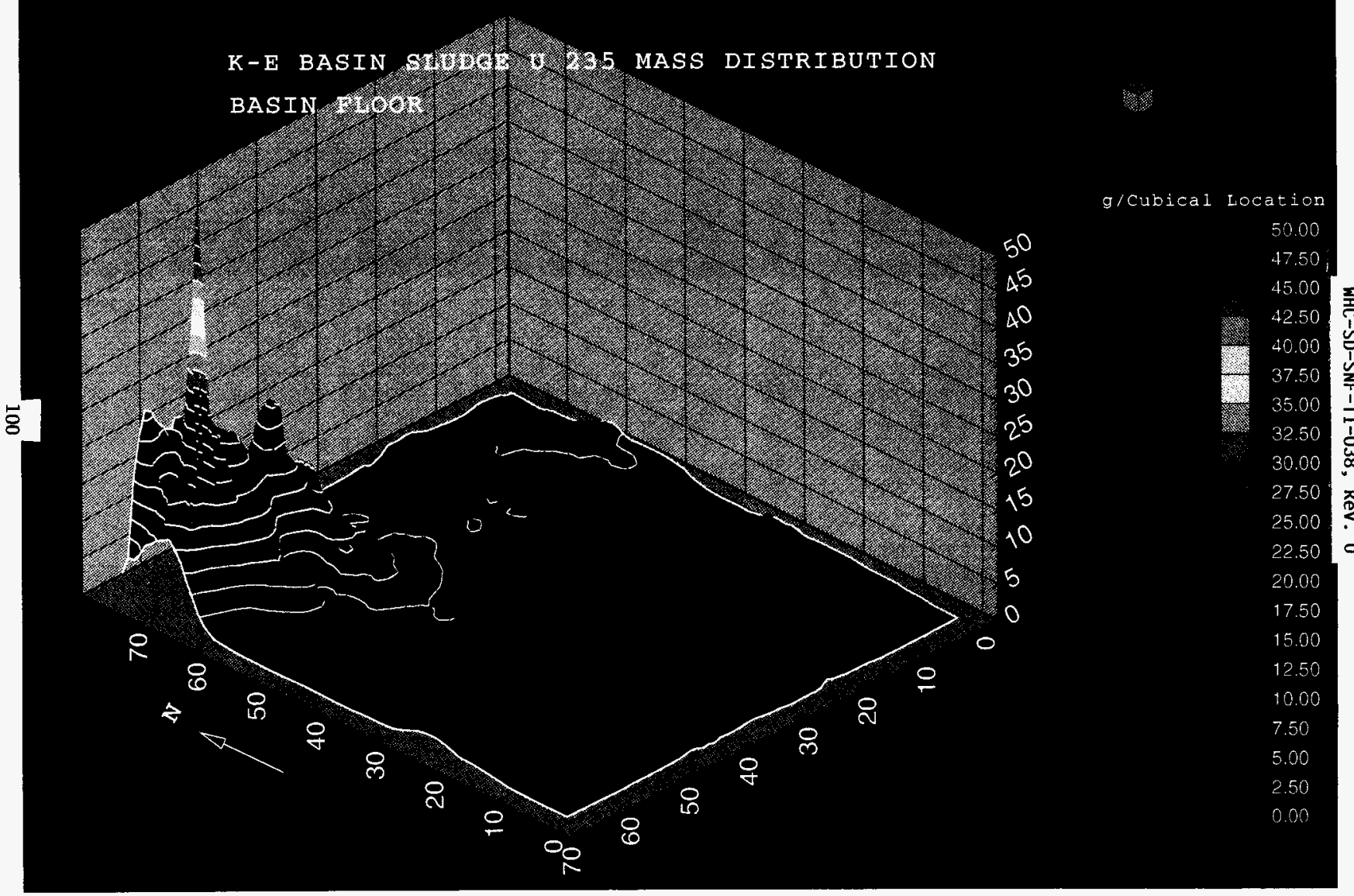




\section{K-E BASIN SLUDGE U 238 MASS DISTRIBUTION}

\section{BASIN FLOOR}

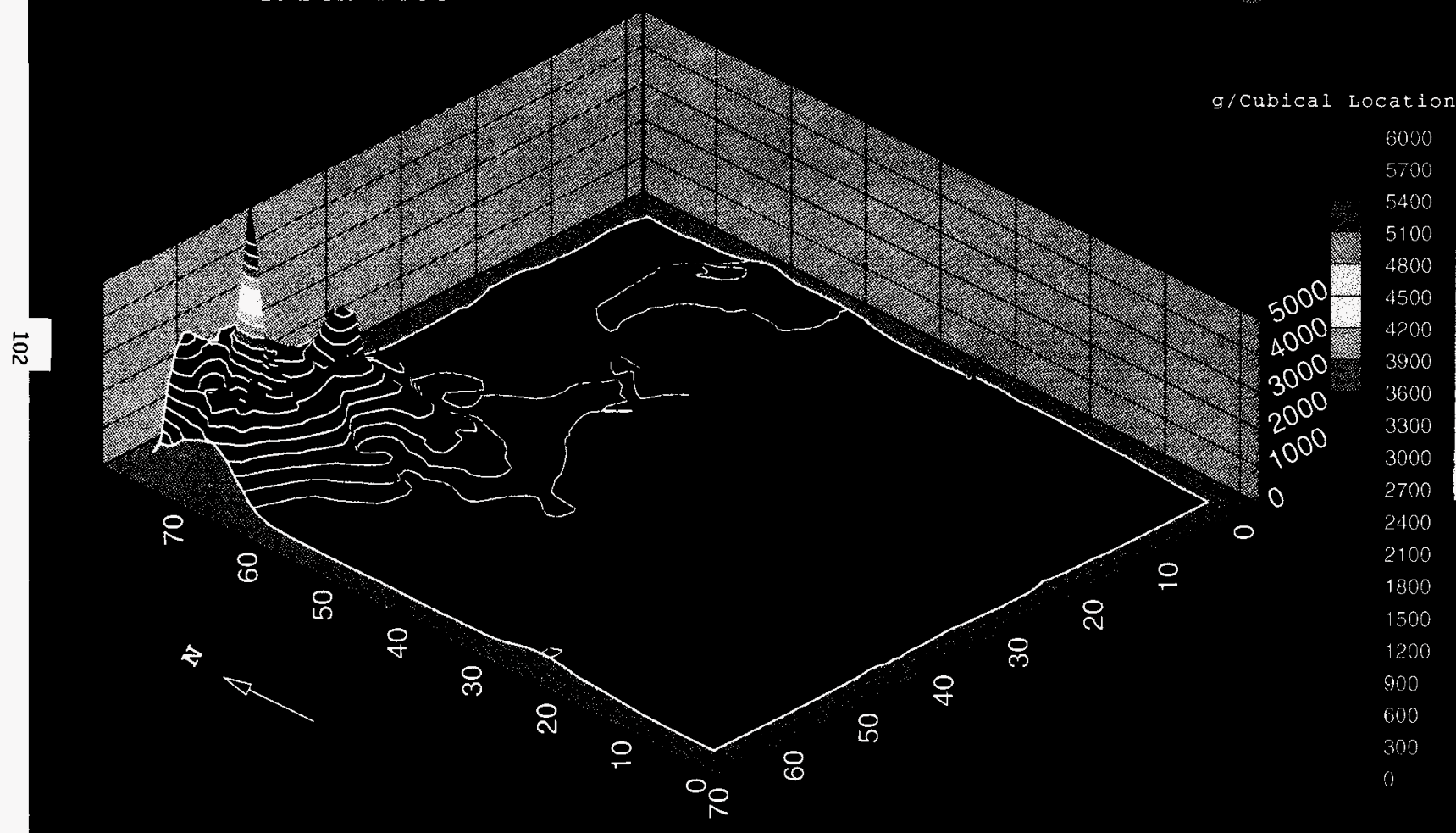


K-E BASIN SLUDGE Ag MASS DISTRIBUTION

\section{BASIN FLOOR}

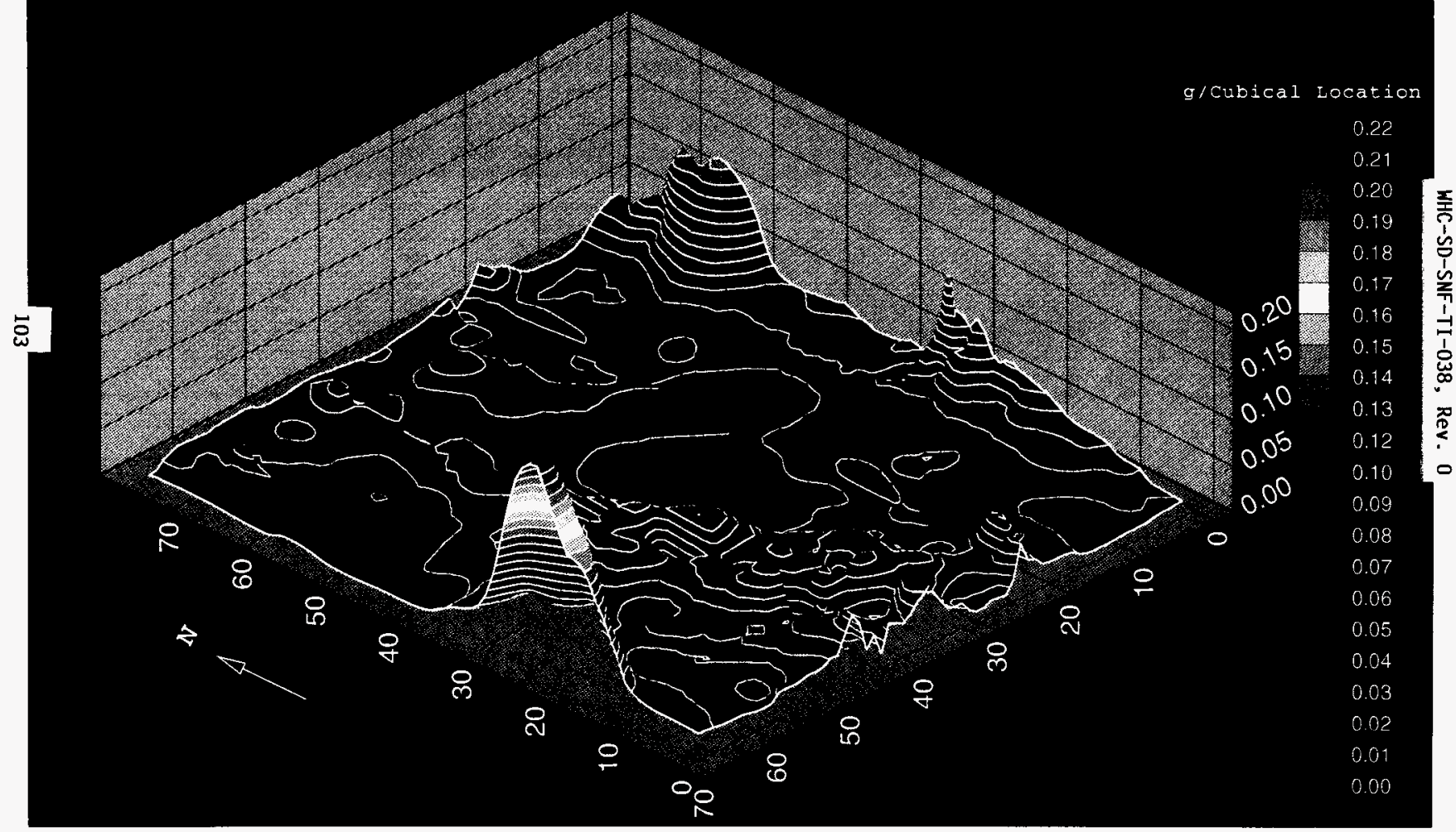




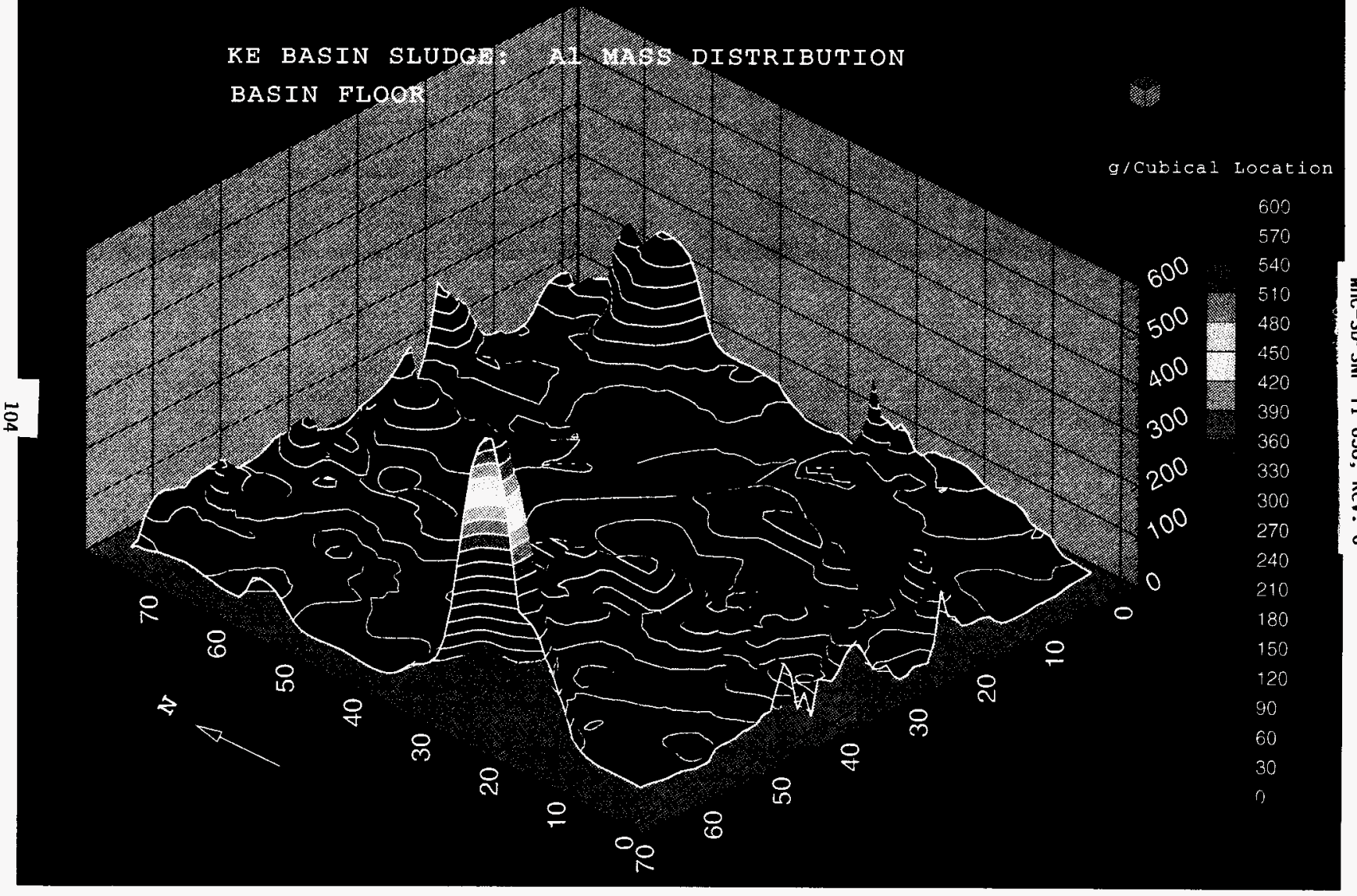


WHC-SD-SNF-TI-038, Rev . 0

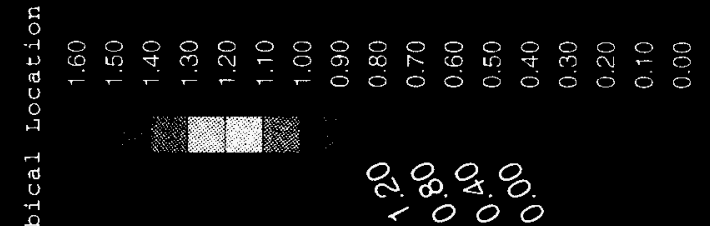

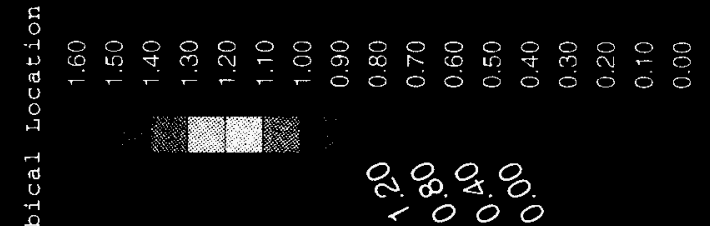

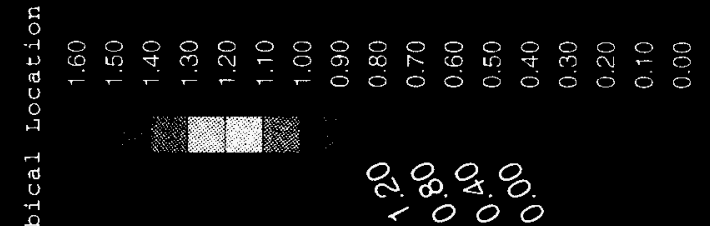

y

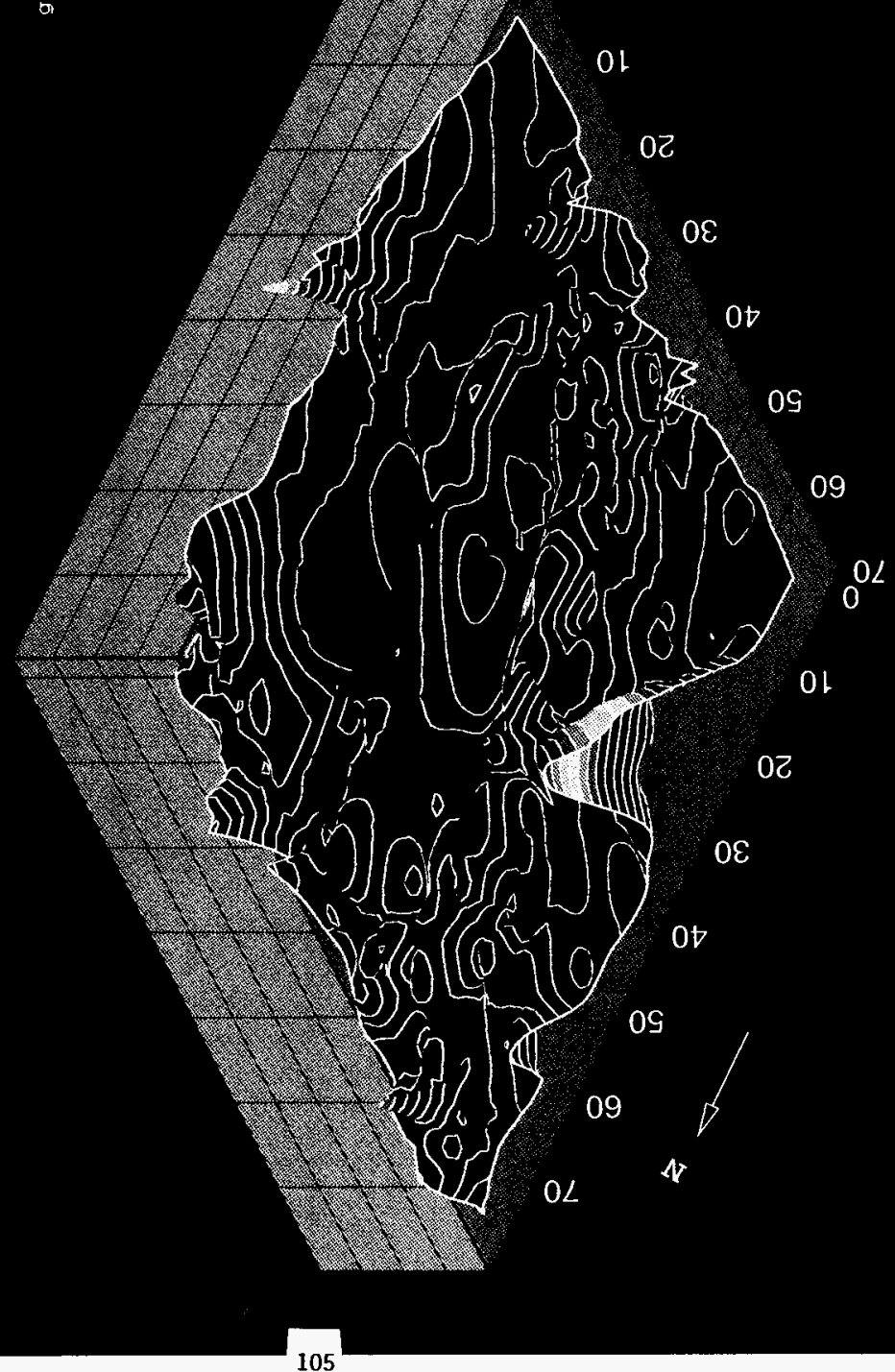


HHC-SD-SNF-TI-038, Rev. 0

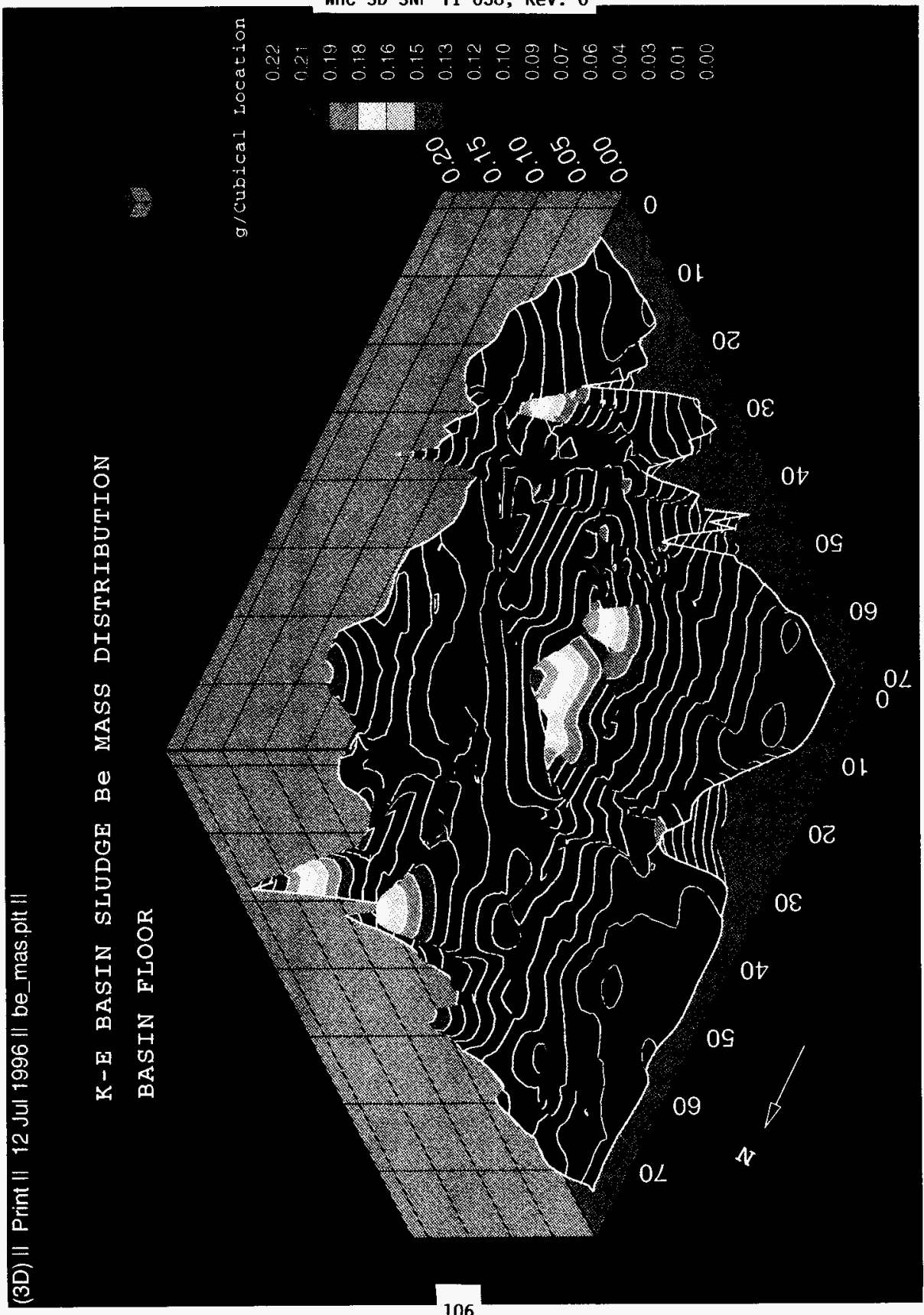




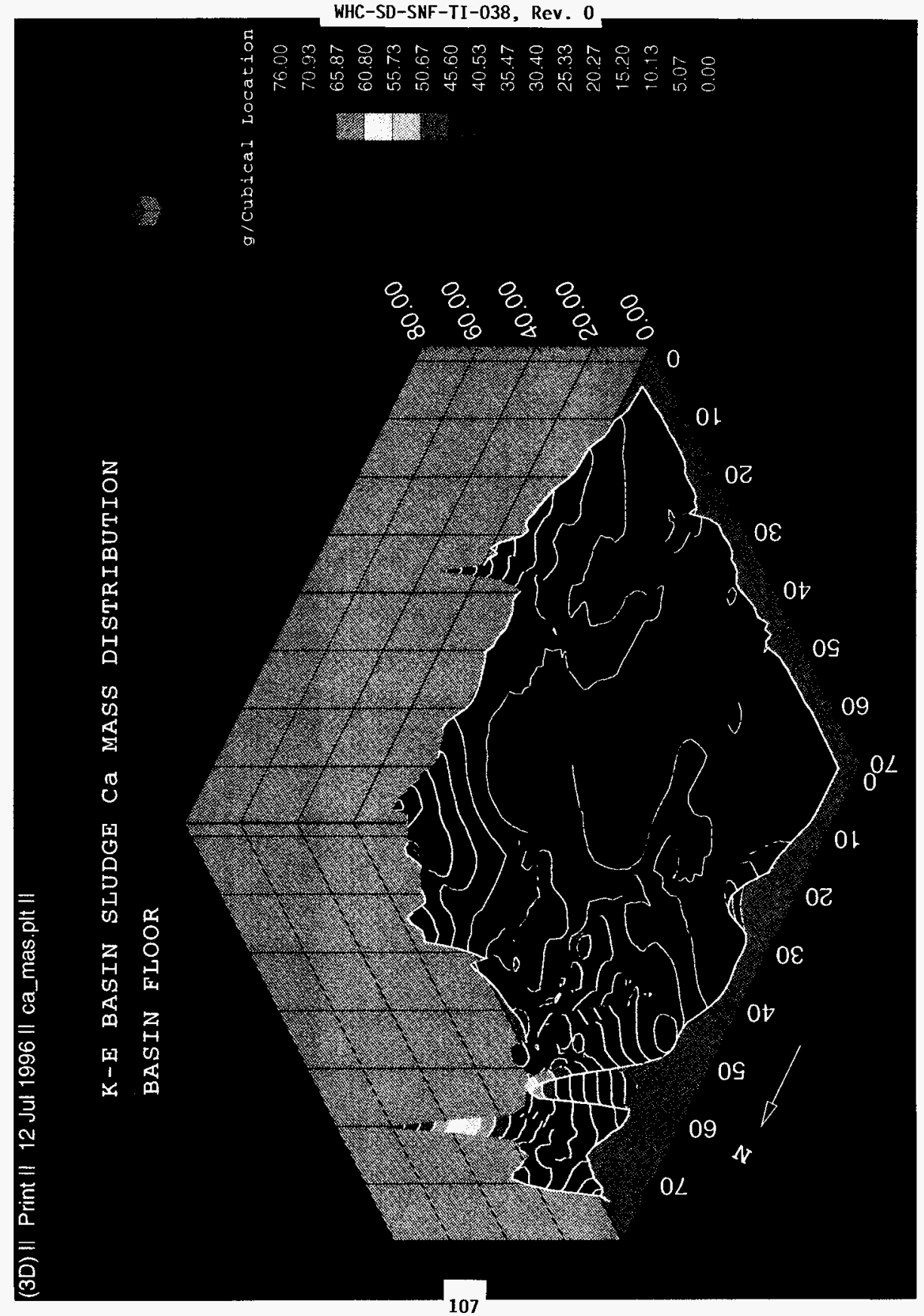



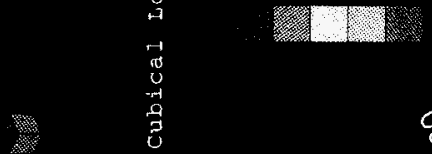

$$
0.9 \% 8 \%
$$

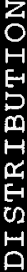
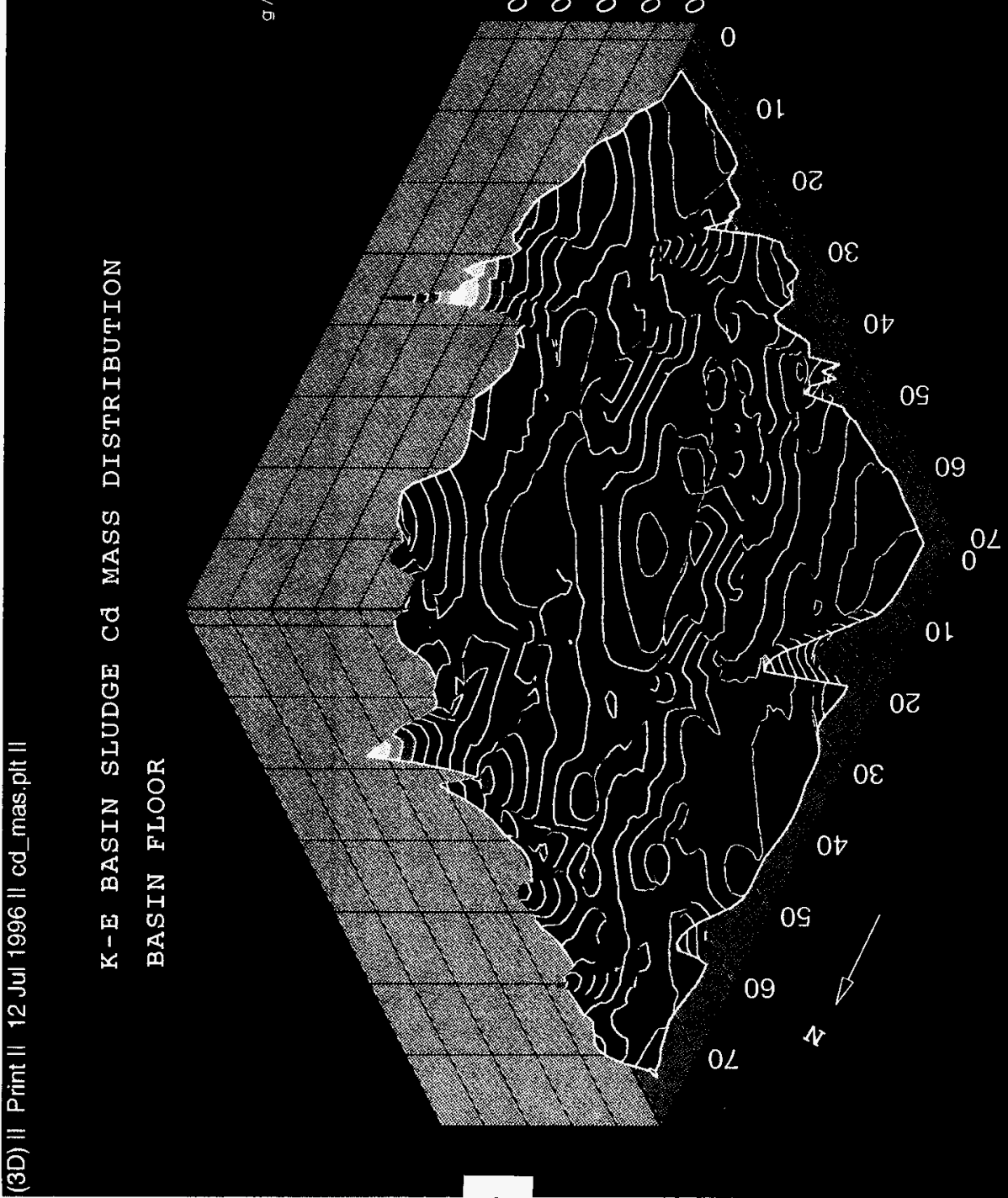
WHC-SD-SNF-TI-038, Rev - 0

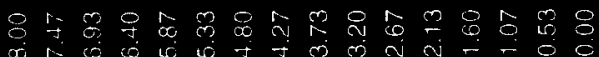

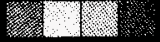

$Z$
$O$
1
$E$
0
0
1
0
0
0
0
0
0
0

$8.8 \% 8$

- 10 - 0
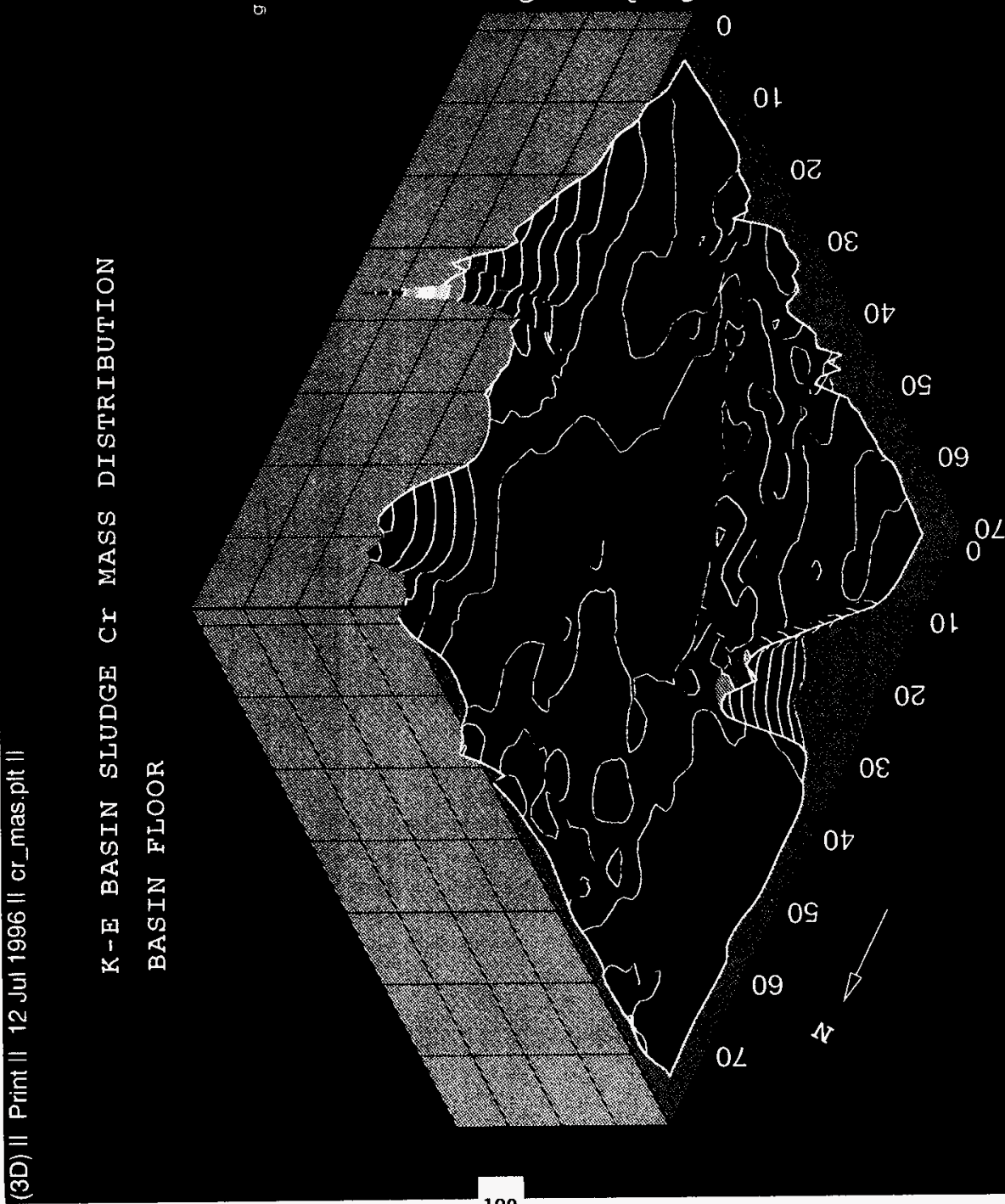


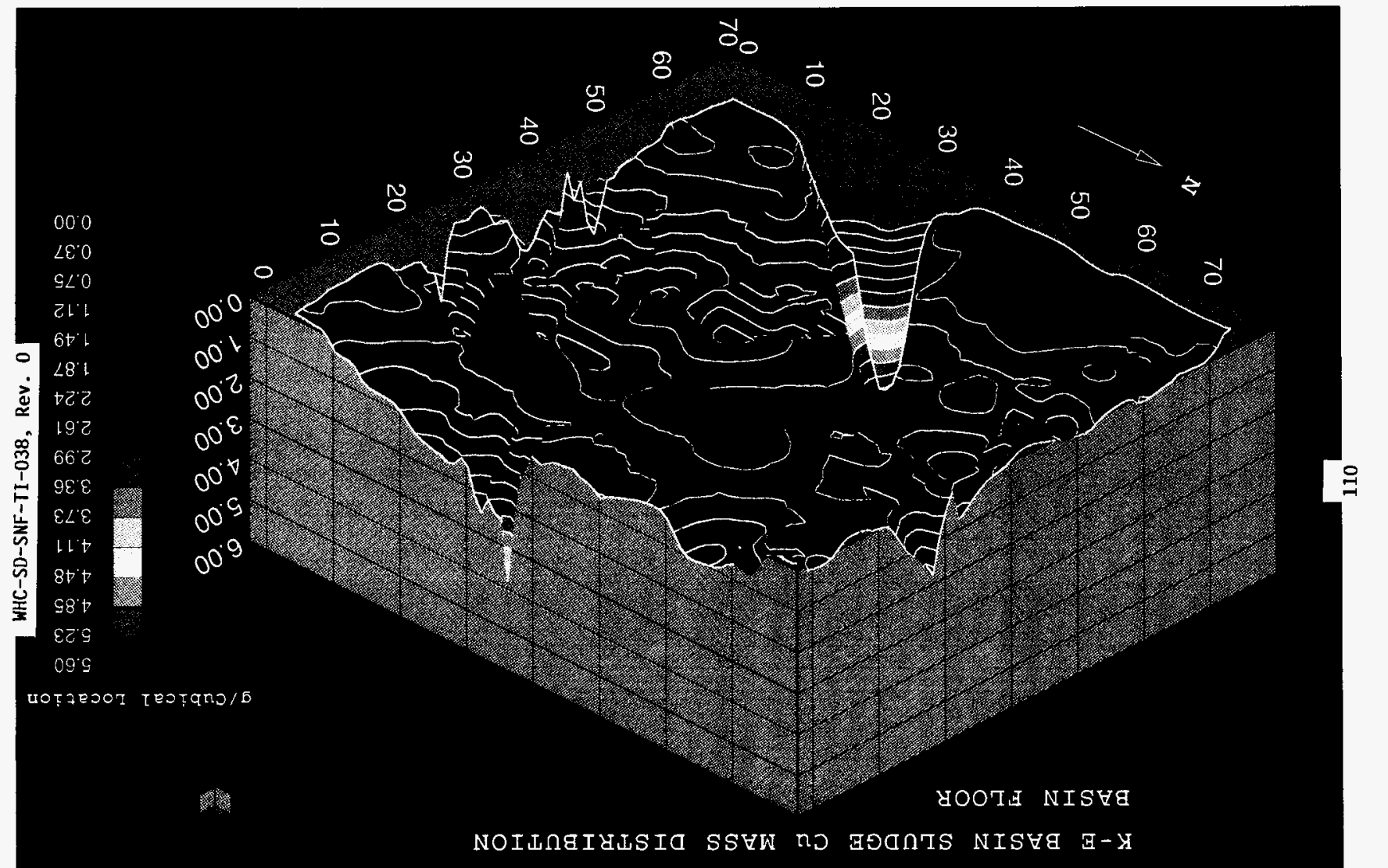


K-E BASIN SLUDGE Fe MASS DISTRIBUTION

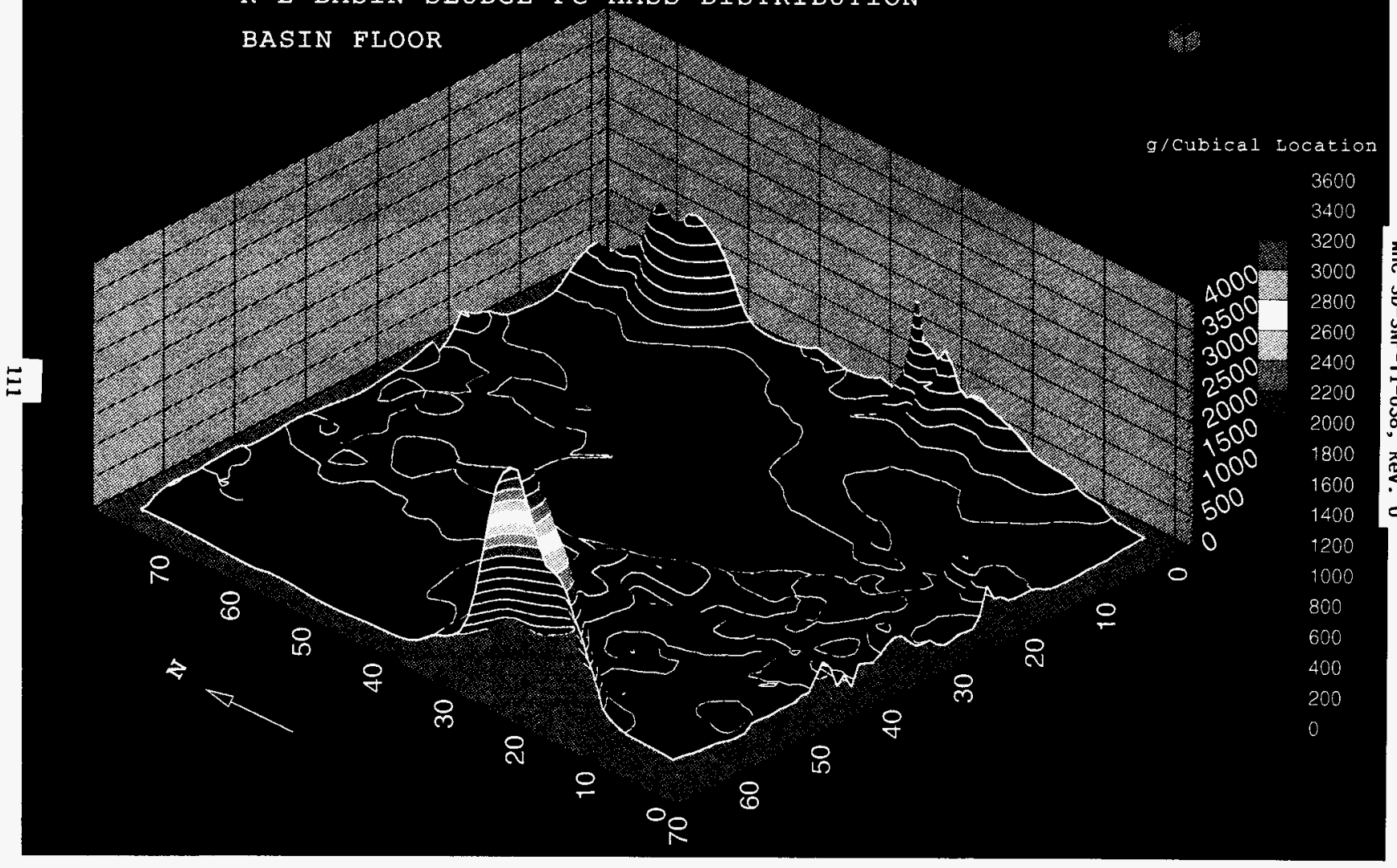




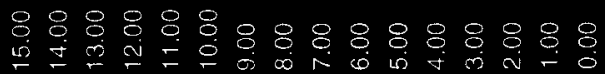

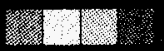

$$
8.8 \% 8 \%
$$

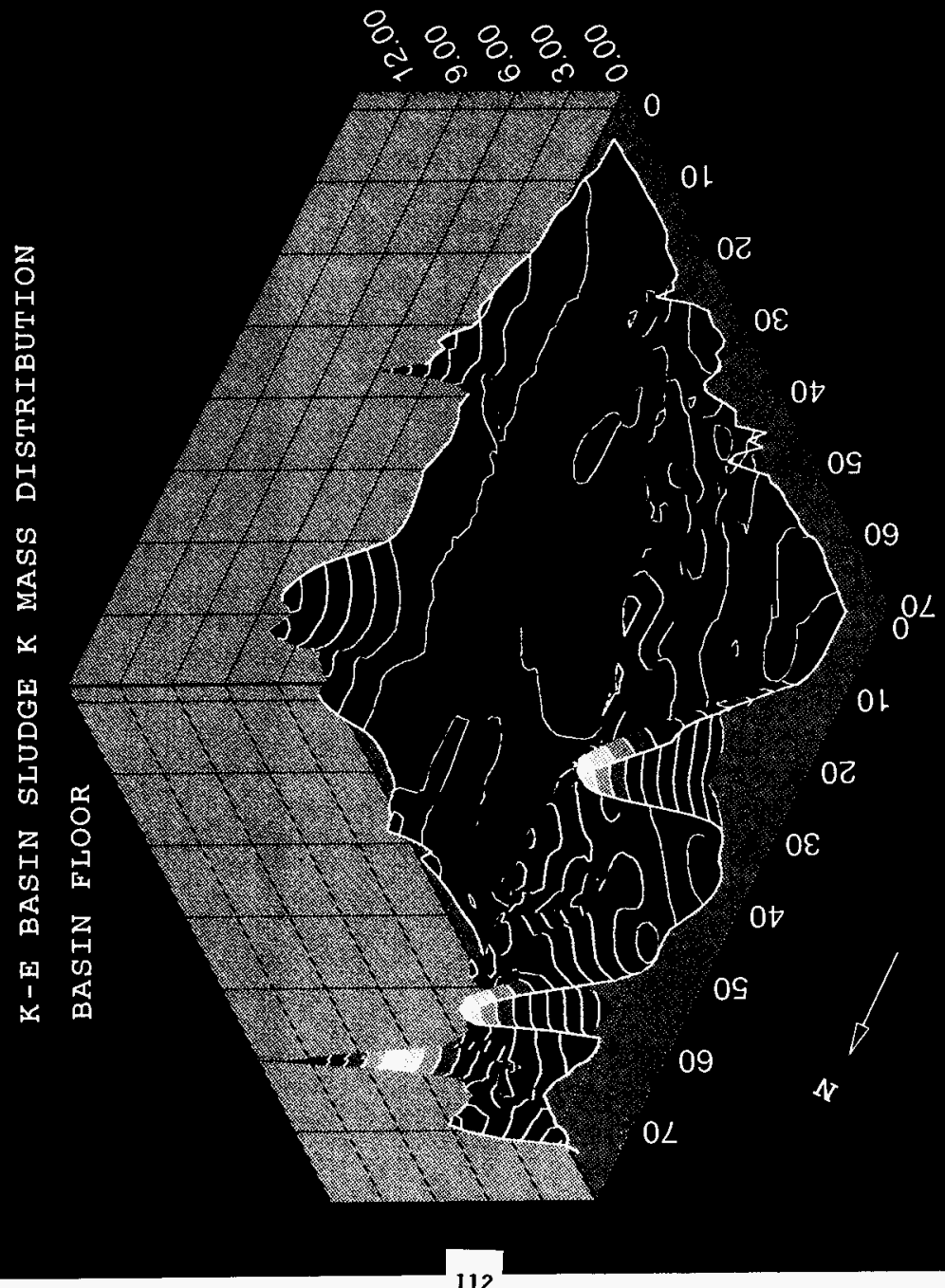


WHC-SD-SNF-TI-038, Rev. 0

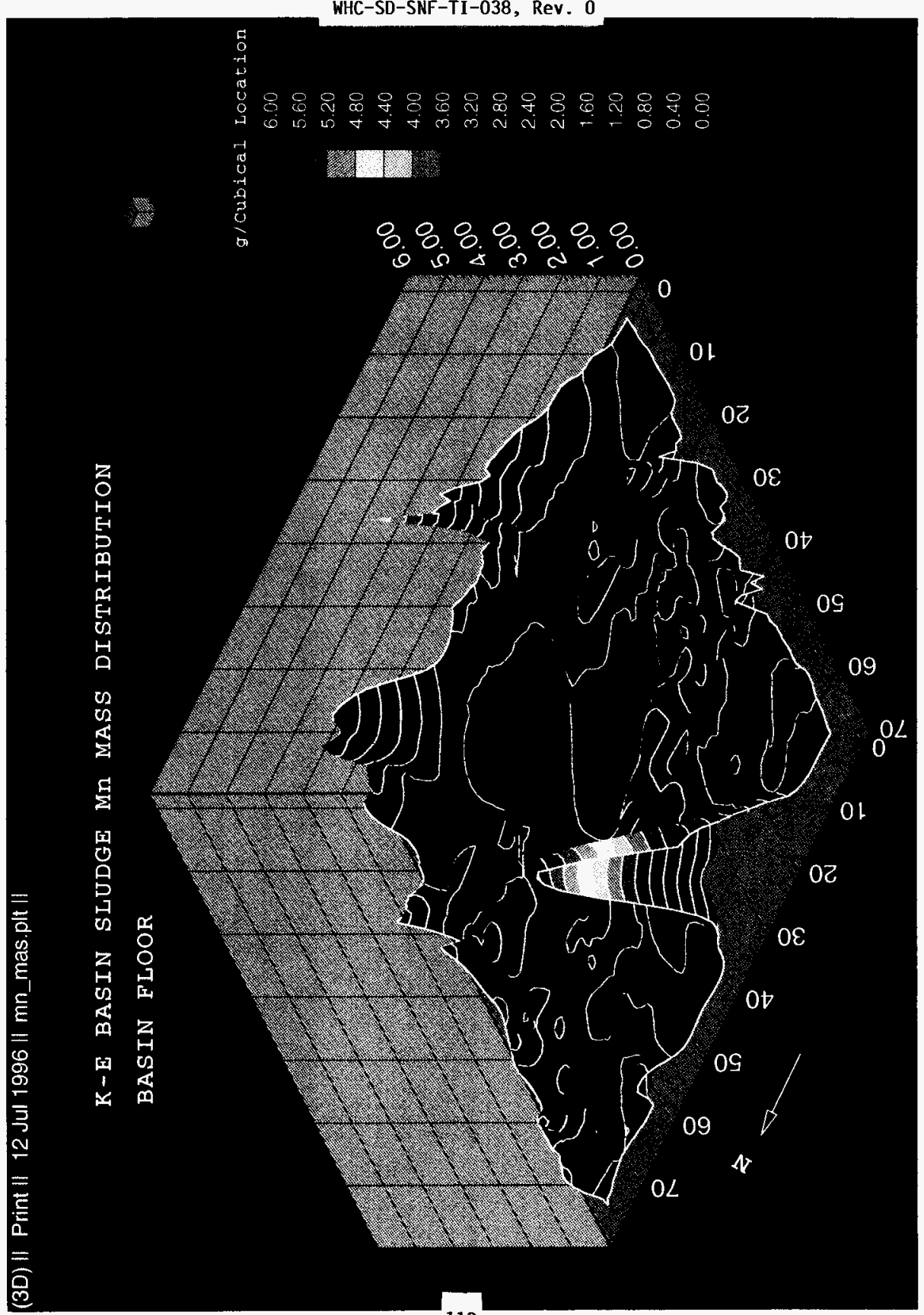




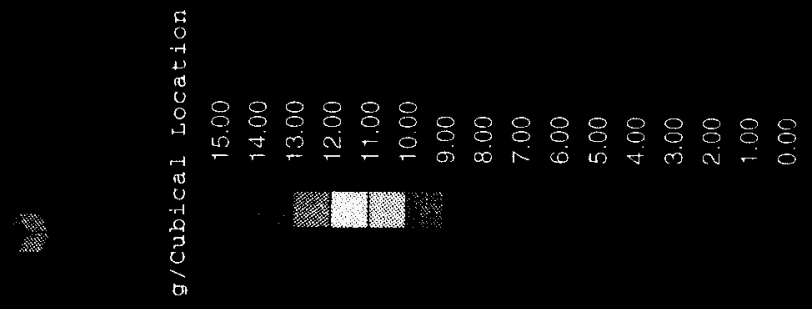

$$
\text { \&. } 8.80 .80
$$

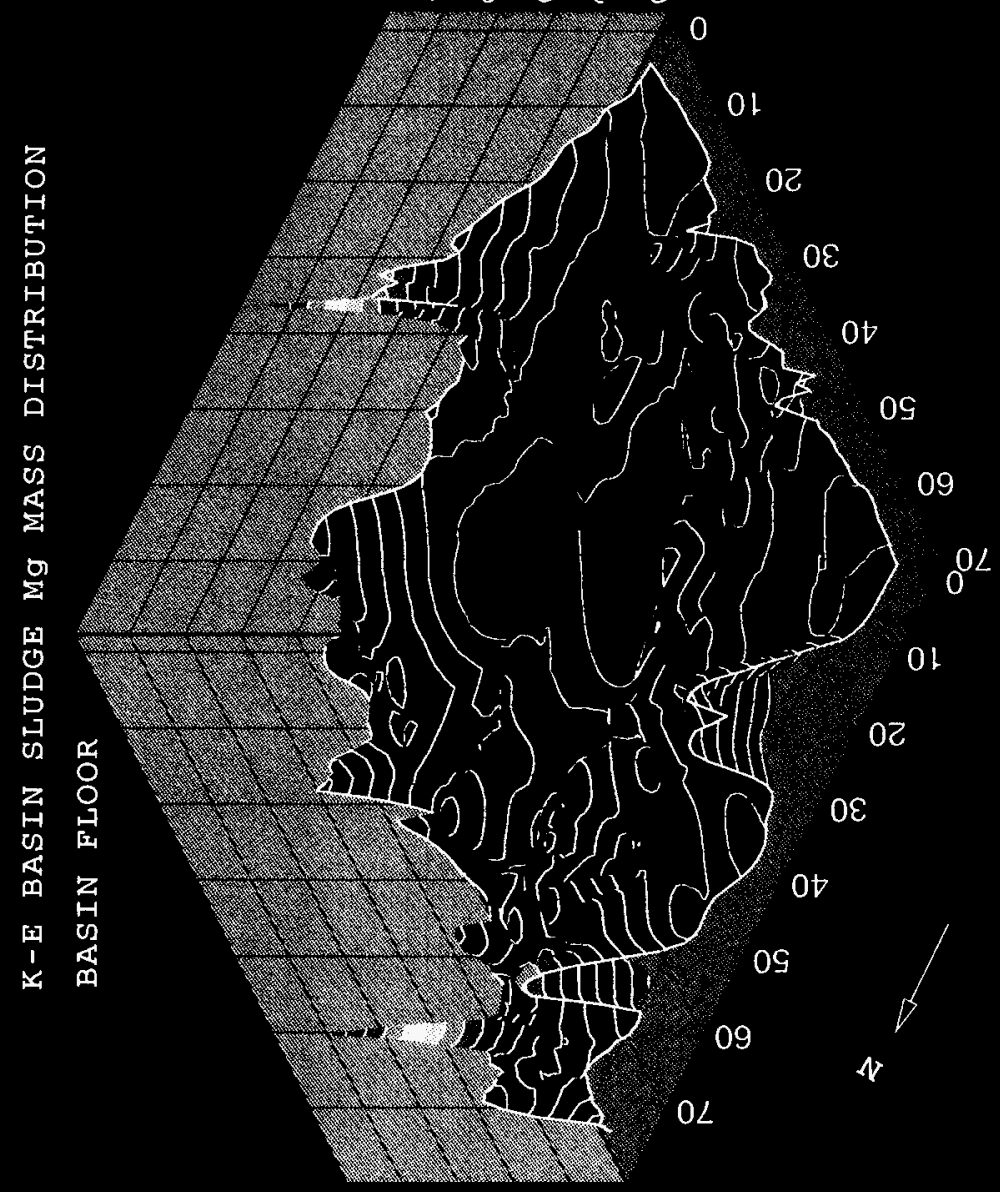




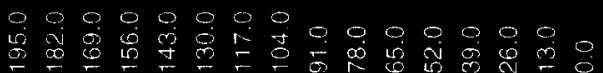

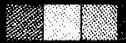

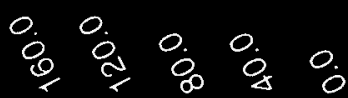

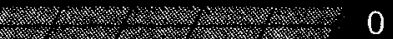

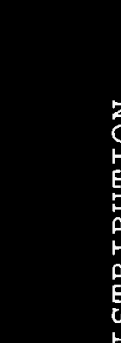



o

U2

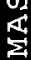

范

且

0

$=\quad$ 早

능

i

$\stackrel{2}{\mathbb{E}}$

E

o 02

든

$\overline{1}$

\&

\%

I 4

3

N

-

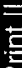
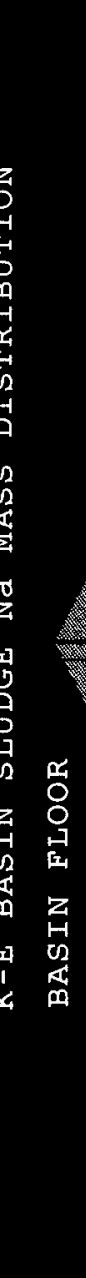

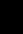 \\ OL}
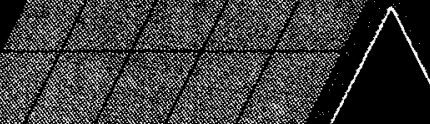
WHC-SD-SNF-TI-038, Rev. 0

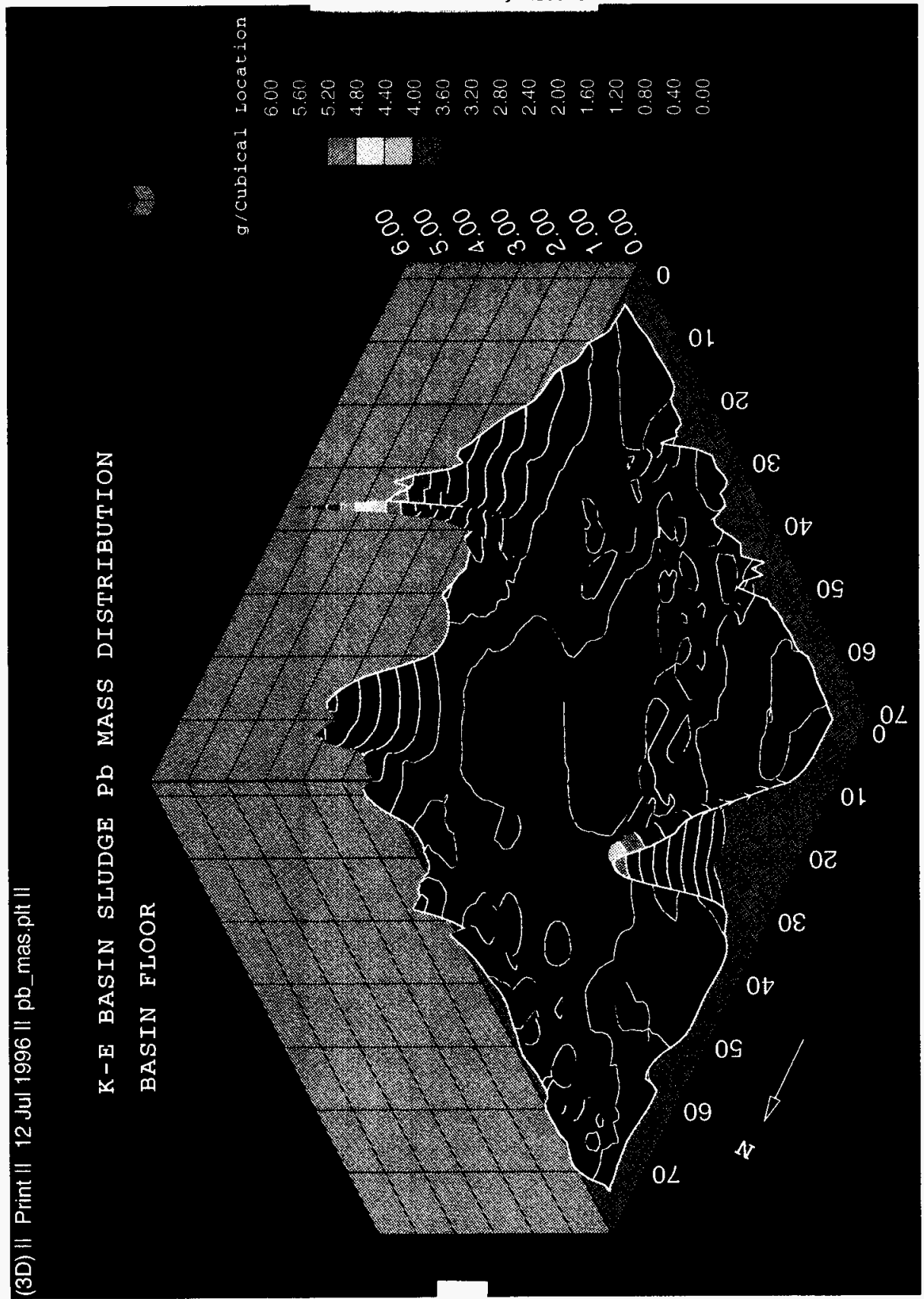


WHC-SD-SNF-TI-038, Rev. 0

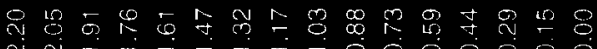

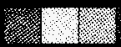

$$
\begin{aligned}
& 8.8 \% 8.8
\end{aligned}
$$

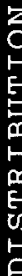

O

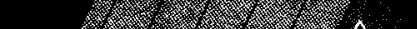
20.70

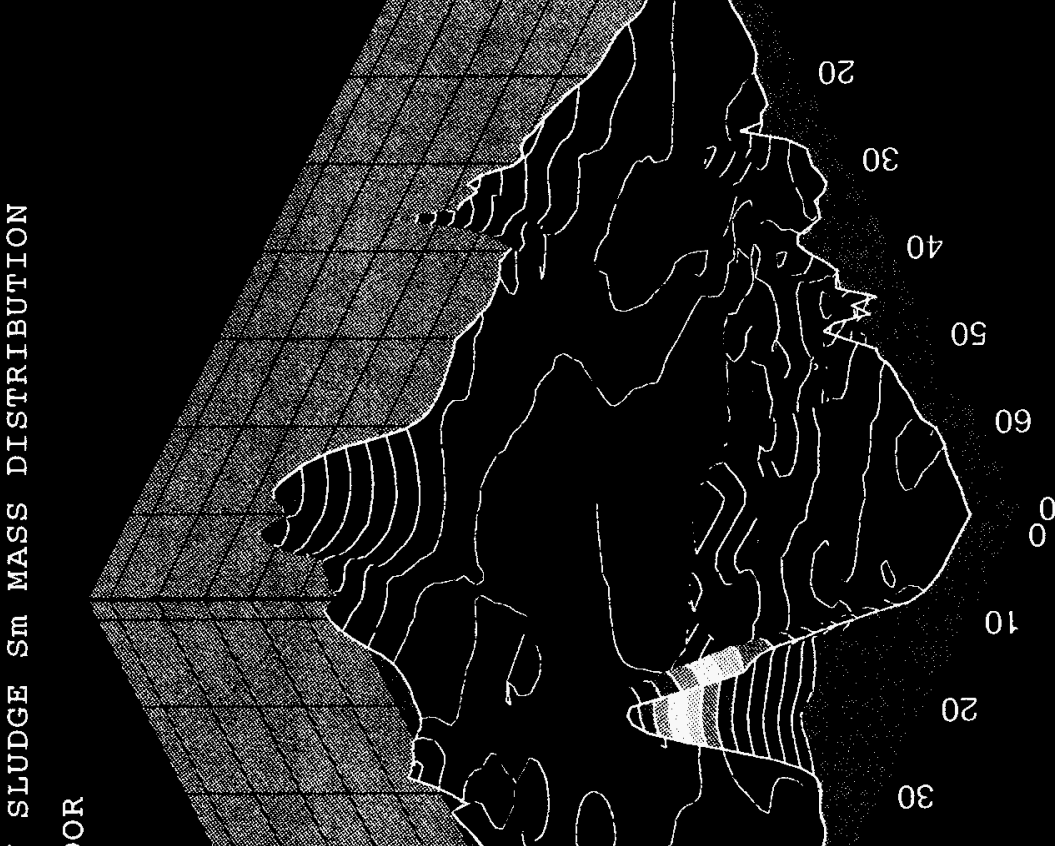




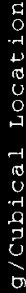

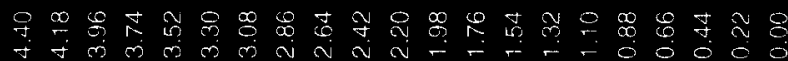

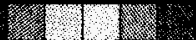

$$
8.8 .8 .8 .8 .8
$$

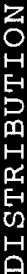

0

C)

E-1

[]

อ

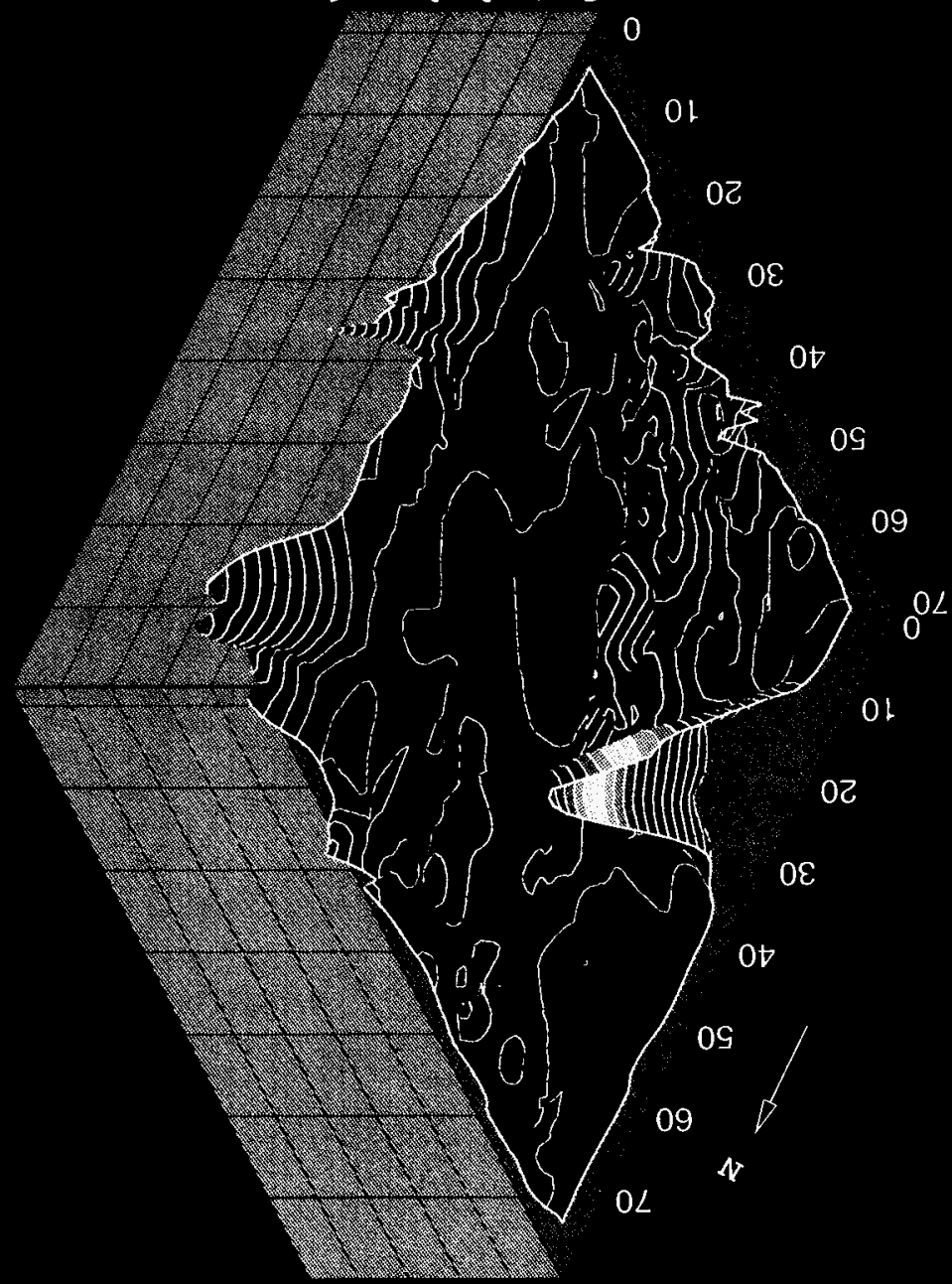


$\oint$
0

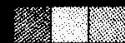

$8,8.88 .8$

0 (

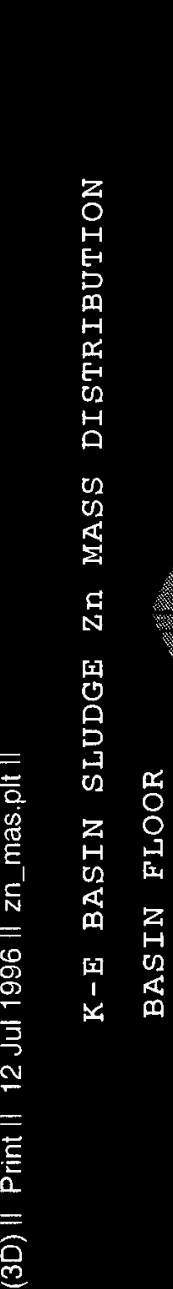

01

02

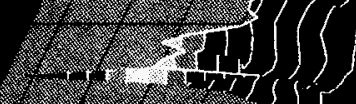

- $\int_{1}^{4}$

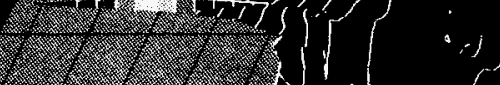

$(2)$
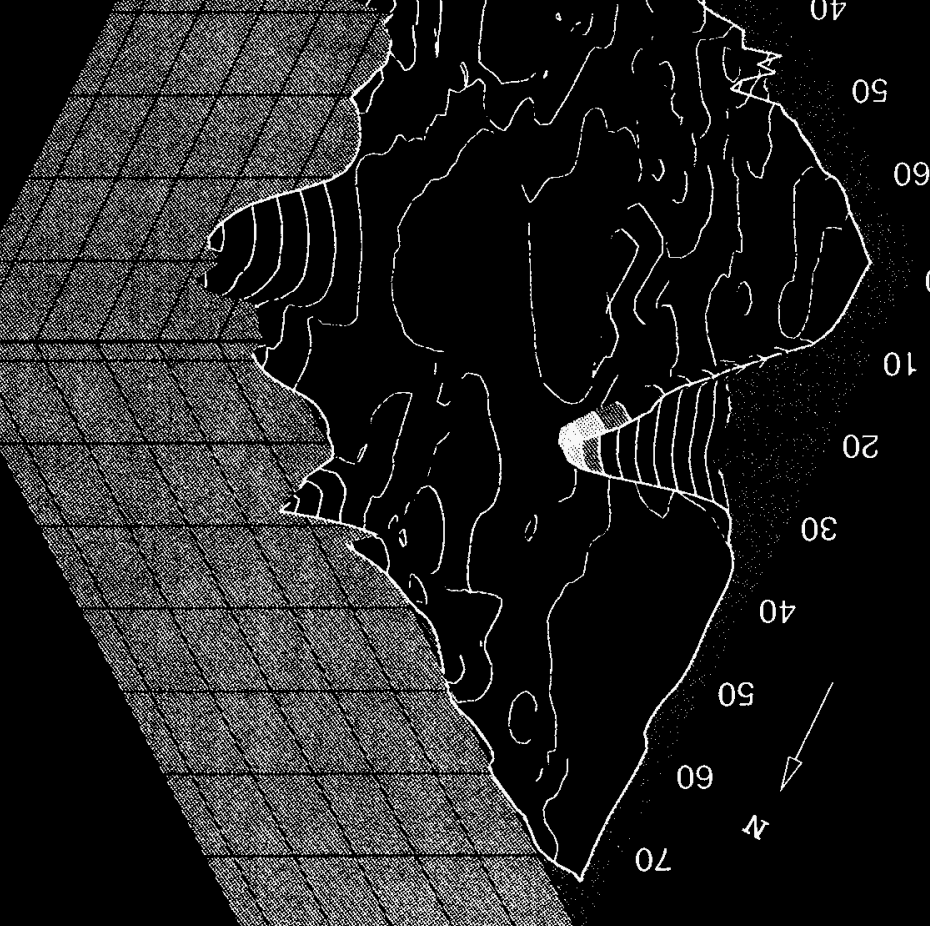

1.

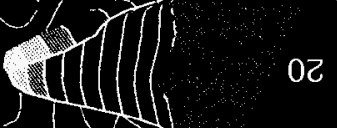




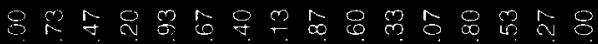

न

(3)

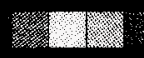

$$
8.8 .8
$$

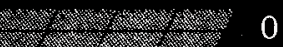

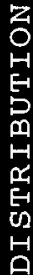

(2)

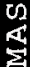

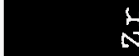

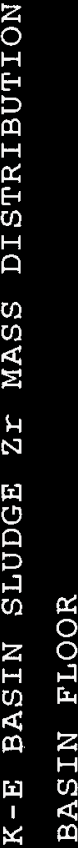

回

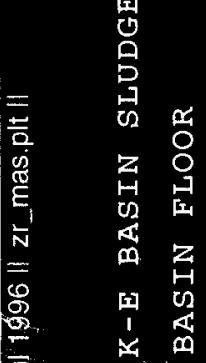

$\overline{3}$

$\cong$

ह

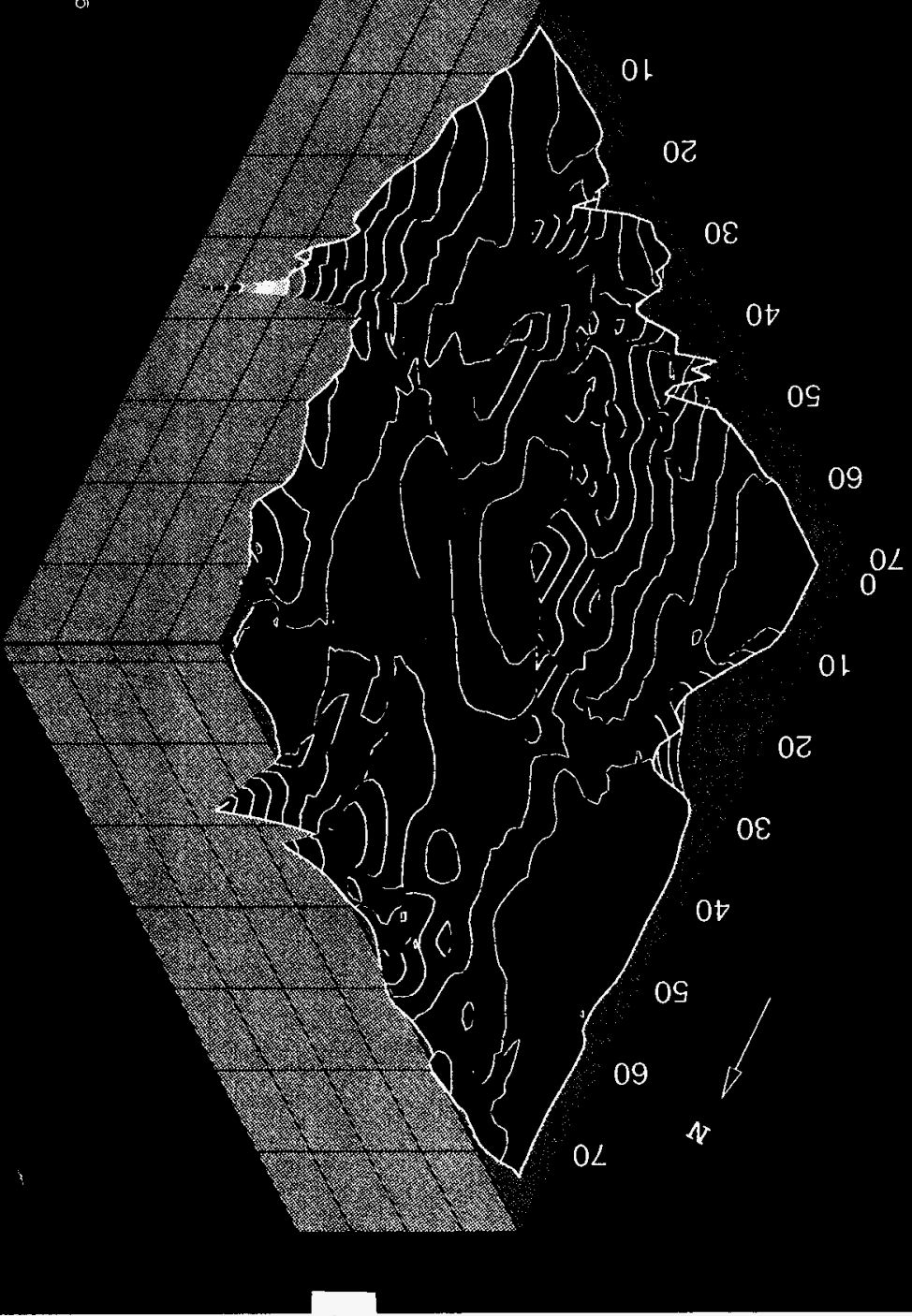


WHC-SD-SNF-TI-038, Rev. 0

$Z$
0
$H$
$E$
0
0
$H$
0
0
0
$1-1$
0
0
0
4
0
2
2
0
0
0
4
0

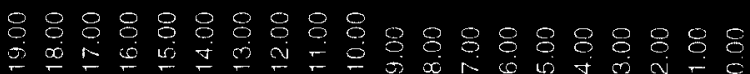

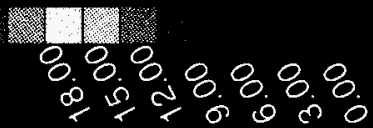

1.

U

1
0
0
0
0

A

E-

O

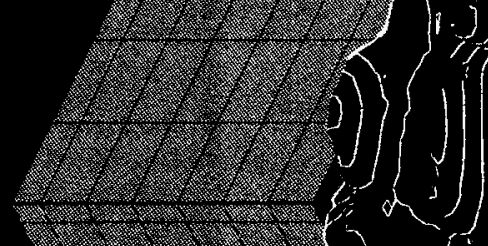

\%)

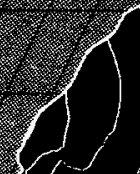

\%
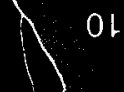

$y \quad 02$
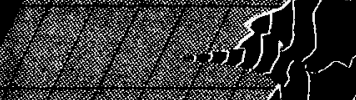

7) $0 \varepsilon$

回

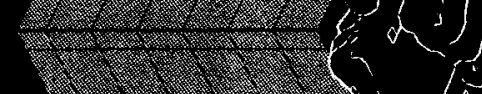

$\int 11$

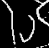

$0 t$

O

Q

$\pm$

등

7

0

jor

$-7 / n$

$-7$

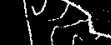

j))
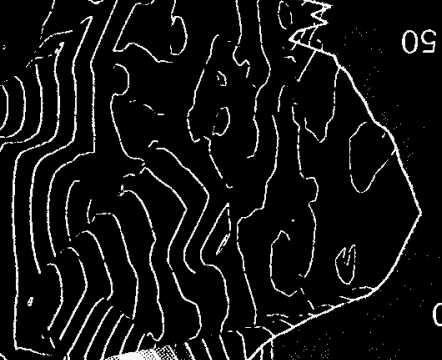

a)

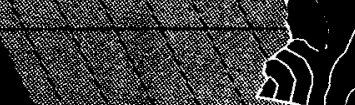

1:m政

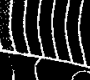

OZ

01

$0 L$

Z

O

0

更

a

i $\quad M$
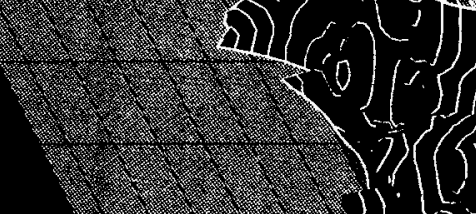

3
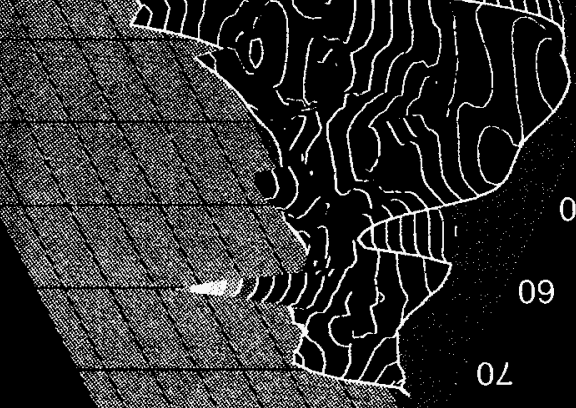

$0 \varepsilon$

ot
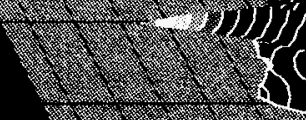

VI,

(1)
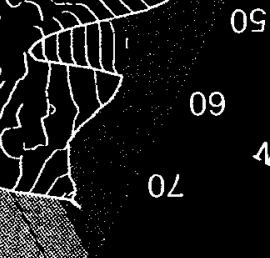

09

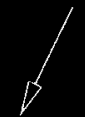

$0 L$

$\checkmark$ 
WHC-SD-SNF-TI-038, Rev. 0

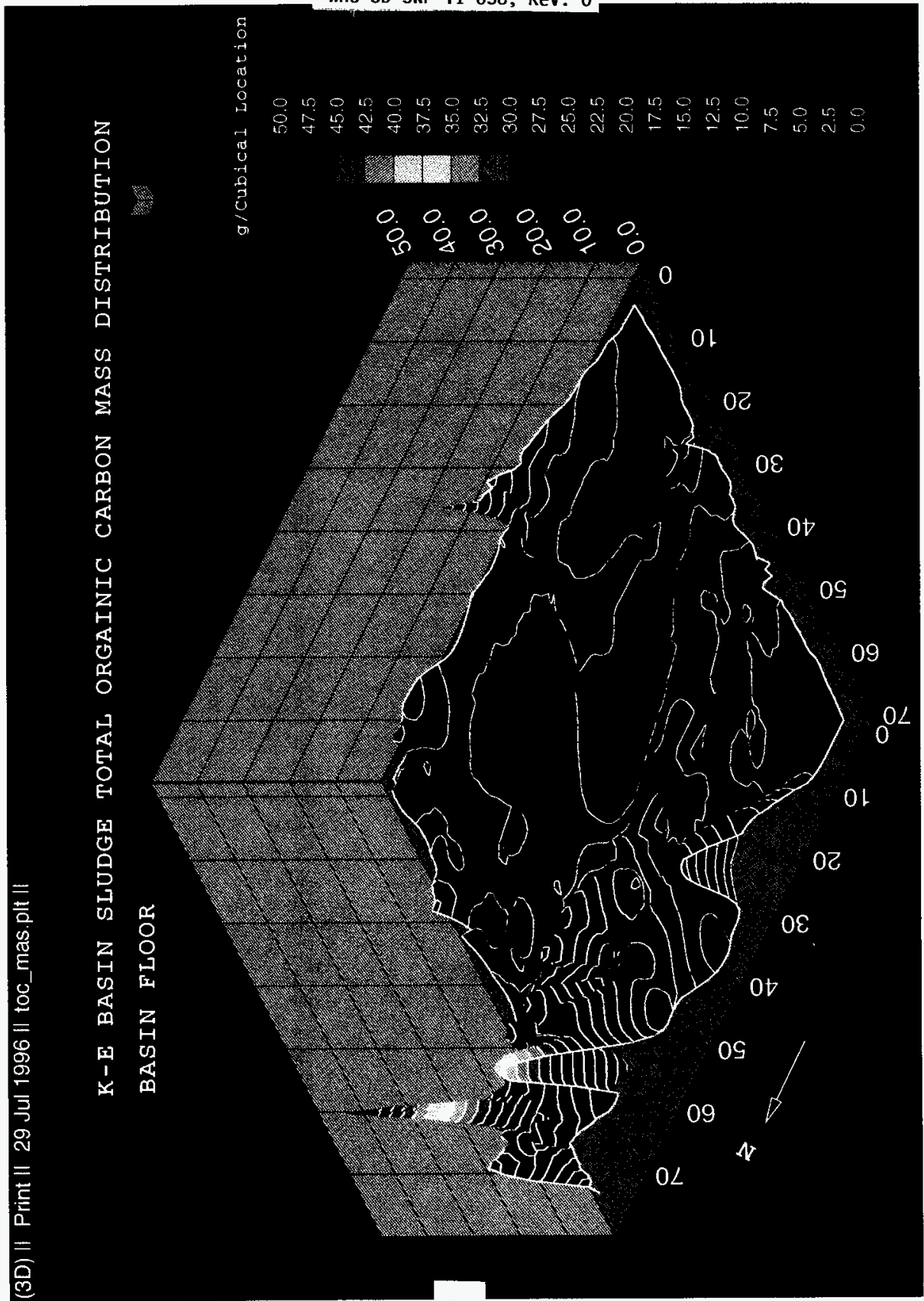


WHC-SD-SNF-TI-038, Rev. 0

APPENDIX D

COMPUTER CODE SOURCE LISTINGS

DWAVG

MASDIS

MASDIS2 
WHC-SD-SNF-TI-038, Rev. 0

This page intentionally left blank. 
WHC-SD-SNF-TI-038, Rev. 0

\section{duavg. $f$}

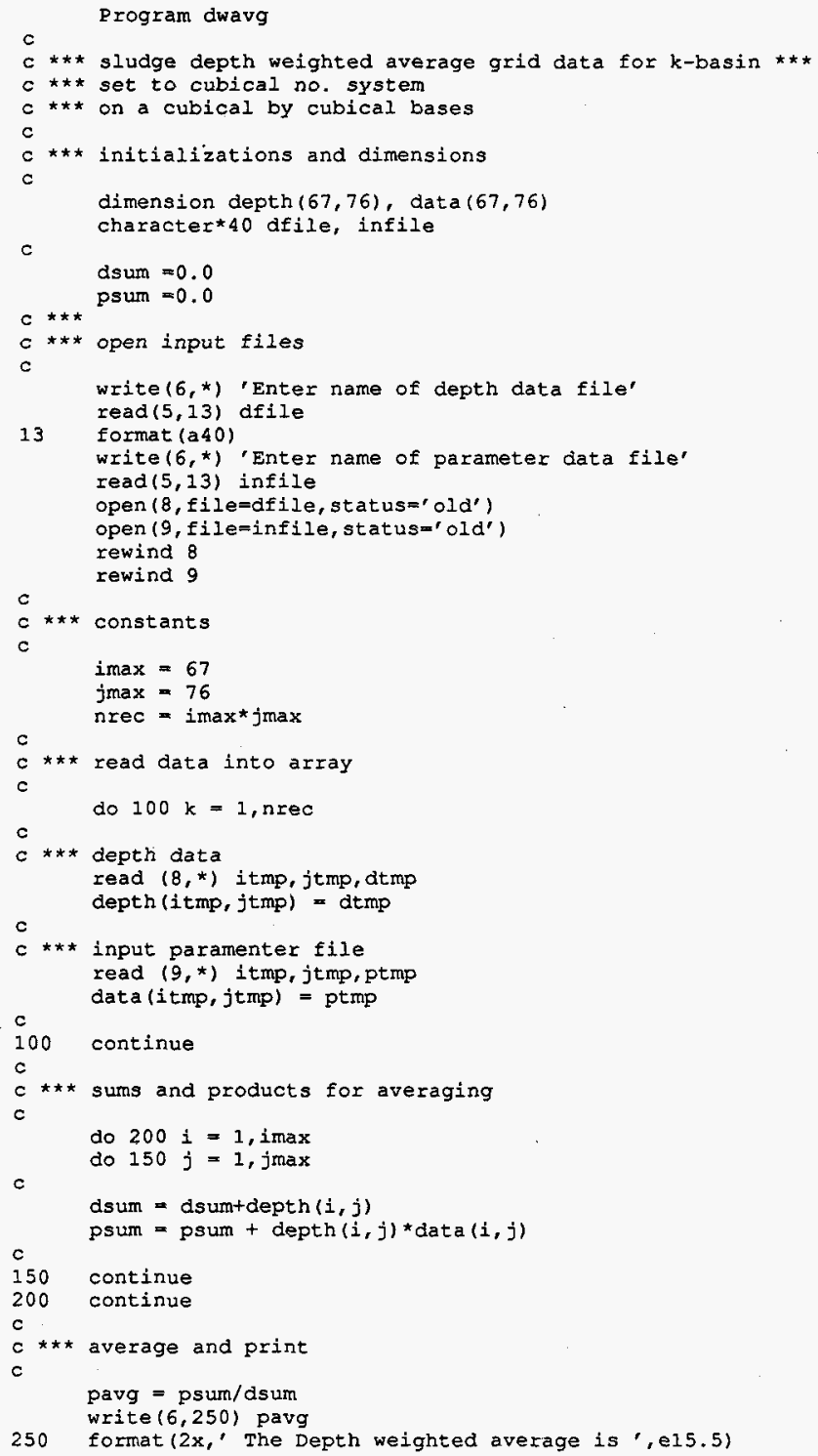

c 
WHC-SD-SNF-TI-038, Rev. 0

dwavg.f

Tue Jun $18 \quad 15: 37: 041996$

2

stop

end 
WHC-SD-SNF-TI-038, Rev. 0

masdis. $f$

Thu Jun $20 \quad 14: 08: 21.1996$

1

Program masdis

$c$

$\star \star *$ sludge mass distibution/grid for elements for k-basin $\star \star *$

$c \star \star \star$ for $\mathrm{g} / \mathrm{mL}$ input

$\star * *$ output in $g /$ cubical

$\star \star *$ set to cubical no. system

$\star \star \star$ on a cubical by cubical bases;

*** initializations and dimensions

$c$

dimension depth $(67,76)$, data $(67,76)$

character 40 dfile, infile, outfile

c

disum $=0.0$

psum $=0.0$

c

area $=881$.

$f a c=$ area* $1 . e-6$

c $\star \star \star *$

$c \star \star \star$ open input files

c

write $(6, *)$ 'Enter name of depth data file'

read $(5,13)$ dfile

13

format $(a 40)$

write $(6, *)$ 'Enter name of parameter data file'

read $(5,13)$ infile

write $(6, *)$ 'Enter name of output data file'

read $(5,13)$ outfile

open (8, file=dfile, status='old')

open $(9$, file=infile, status='old')

open $(10$, file=outfile)

rewind 8

rewind 9

rewind 10

$\mathrm{c}$

c *** constants

c

$i \max =67$

$j \max =76$

nrec $=i \max * j \max$

$c$

$c * \star$ read data into array

c

do $100 \mathrm{k}=1, \mathrm{nrec}$

$c$

c $\star \star \star$ depth data

read $(8, \star)$ itmp, jtmp, dtmp

depth (itmp, jtmp) = dtmp

$\mathrm{c}$

c *** input paramenter file

read $(9, *)$ itmp, jtmp, ptmp

data (itmp, jtmp) $=$ ptmp

100 continue

c

$c \star \star \star$ cal mass/locations, (i,j index)

c

do $200 i=1$, imax

do $150 j=1, j \max$

$c$

tot $=\operatorname{depth}(i, j) \star \operatorname{data}(i, j) \star \mathrm{fac}$

c

write $(10,201)$ i,j,tot

$c$ 
WHC-SD-SNF-TI-038, Rev. 0

masdis.f

Thu Jun $20 \quad 14: 08: 21.1996$

2

150 continue

200 continue

$c$

201 format $(2 i 5, e 12.4)$

c

stop

end 
WHC-SD-SNF-TI-038, Rev. 0

Program masdis2

$c$

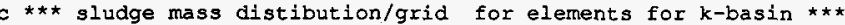

$c \star \star *$ for micro $\mathrm{Ci} / \mathrm{mL}$ input

$\star \star \star$ output in $\mathrm{g} / \mathrm{cubical}$

c $\star \star \star$ set to cubical no. system

$c \star \star \star$ on a cubical by cubical bases

$c \star \star \star$ initializations and dimensions

$c$ dimension depth $(67,76)$, data $(67,76)$

$c$ character* 40 dfile, infile, outfile

dsum $=0.0$

psum $=0.0$

$c$

area $=881$.

fac $=$ area* $1 \cdot e-6$

$c \star \star \star$

$c * \star \star$ open input files

$c$

write $(6, *)$ 'Enter name of depth data file' read $(5,13)$ dfile

13

format (a40)

write $\left(6,{ }^{*}\right)$ 'Enter name of parameter data file'

read $(5,13)$ infile

write $(6, *)$ 'Enter name of output data file'

read $(5,13)$ outfile

open $(8, f i l e=d f i l e$, status='old')

open $(9, f i l e=i n f i l e$, status="old')

open $(10$, file=outfile)

rewind 8

rewind 9

rewind 10

$c$

$\star \star \star \star$ constants

$c$

write $(6, *)$ 'Enter conversion factor for isotope Ci to grams' read $(5, *)$ confac

fac $=f a c * c o n f a c$

$c$

$i \max =67$

$j \max =76$

nrec $=i \max * j \max$

$c$

$c \star \star \star$ read data into array

c

do $100 \mathrm{k}=1$, nrec

$c$

$c \star \star \star$ depth data

read $\left(8,{ }^{*}\right)$ itmp, jtmp, dtmp

depth (itmp, jtmp) = dtmp

$c$

c $* * \star$ input paramenter file

read $(9, *)$ itmp, jtmp, ptmp

data (1tmp, jtmp) = ptmp

$c$

100 continue

$c$

$c \star \star \star$ cal mass/locations, (i,j index)

$c$

do $200 i=1$, imax

do $150 j=1$, jmax 
WHE-SD-SNF-TI-038, Rev. 0

masdis2, $f$

Tue Aug $27 \quad 12: 21: 501996$

2

tot $=\operatorname{depth}(i, j) \star \operatorname{data}(i, j) \star f a c$

c

write $(10,201) i, j$, tot

150 continue

200 continue

201 format (2i5, e12.4)

c

stop

end 
WHI:-SD-SNF-TI-038, Rev. 0

This page intentionally left blank. 


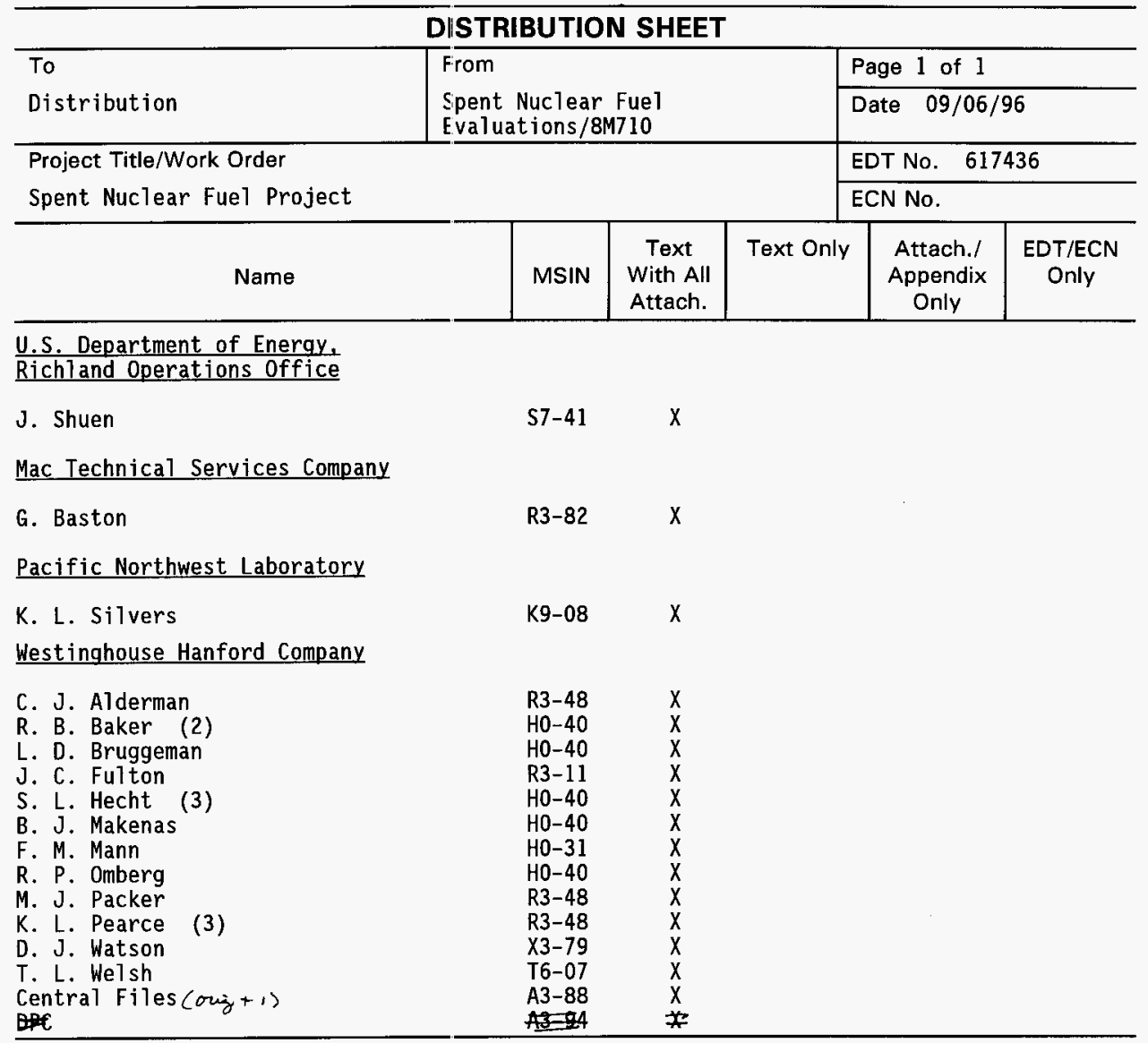

